\title{
DISSIPATIVE HYPERBOLIC SYSTEMS
}

BY

\section{R. S. PHILLIPS( ${ }^{(1)}$}

1. Introduction. This paper is concerned with the solution of the Cauchy problem for dissipative hyperbolic systems of linear partial differential equations for the case of one spatial variable and time-invariant coefficients. Here we use the term dissipative to mean that the associated physical model has no internal energy sources; if, in addition, the boundary conditions are such that no energy enters the model through the boundary, then the solution is called dissipative. By employing the theory of semigroups of linear bounded operators, we are able to obtain all possible dissipative semi-group solutions without further restricting the behavior of the differential system near the boundary. In order that our considerations include all of the dissipative boundary conditions commonly associated with hyperbolic systems, we have also treated the above differential system coupled at the end points to dissipative systems having finite degrees of freedom.

The semi-group method is in essence an abstract analogue of the classical Laplace-transform treatment of the Cauchy problem. From this point of view the present paper can be thought of as an extension of a time honored development going back to the works of M. Plancherel [14], G. Doetsch [2], and W. Mächler [9], to mention a few of the early publications on the wave equation. The above papers deal with the regular case; that is, finite domains and sufficiently smooth coefficients. More recently, G. Hellwig [6] and K. Yosida $[16 ; 17]$ have treated the Cauchy problem for the wave equation allowing certain kinds of singular behavior at the boundary. Because of the difference in settings, the Hellwig and Yosida developments cannot readily be compared with the present work; however, neither of these papers considers boundary behavior or boundary conditions as general as those treated here.

With regard to method, perhaps the feature which best sets the present study off from those mentioned above, is the central role played by the energy integral. The use of the energy integral in dealing with the wave equation is, of course, not new. In fact, as early as 1900, J. Hadamard [5] employed the energy integral to establish uniqueness for the solution of the mixed problem. Later, K. O. Friedrichs and H. Lewy [4] discovered another

Presented to the Society August 21, 1956; received by the editors July 25, 1956.

(1) This paper was written under the sponsorship of the Office of Naval Research, U. S. Navy, under contract Nonr 228(09). 
pertinent property of the energy integral. They noted that the solution of the wave equation with two or more spatial variables did not always continue the smoothness properties of the initial data; for example, it is possible for the solution to have a caustic surface even when the initial data does not. Nevertheless, Friedrichs and Lewy were able to show that the finiteness of the energy integral is continuable in the above sense. For the one spatial variable case considered in the present paper, this property of the energy integral turns out to be a simple consequence of the semi-group property of the solution. Finally it should be pointed out that inequalities derived from the energy integral are the basis of most existence theorems for the solution of linear, and even nonlinear, hyperbolic partial differential equations. In this connection, we mention the recent paper by K. O. Friedrichs [3], where further references may be found.

We return now to the problem at hand, that is, the consideration of dissipative hyperbolic linear partial differential systems in one spacial variable of the form

$$
E y_{t}=(A y)_{x}+B y, \quad-\infty \leqq a<x<b \leqq \infty, 0<t .
$$

Here $y=\left(\eta^{1}, \eta^{2}, \cdots, \eta^{k}\right)$ is a $k$-dimensional vector-valued function of $x$ and $t ; E, A, B$ are $k \times k$ matrix-valued functions of $x$ alone, $E$ being hermitian positive definite, $A$ hermitian and of constant rank $r$, and $B$ satisfying the condition $\left({ }^{2}\right)$

$$
B+B^{*}+A_{x} \leqq \Theta, \quad a<x<b,
$$

where $B^{*}$ is the adjoint matrix to $B$ under the assumed inner product

$$
(y, z)=\sum_{i=1}^{k} \eta^{i} \bar{\zeta}^{i} \text { with }|y|=[(y, y)]^{1 / 2} .
$$

In addition it is assumed that the elements of $E$ and $A$ are absolutely continuous $\left({ }^{3}\right)$ on each compact subinterval of $(a, b)$, and that the elements of $E_{x}, A_{x}$, and $B$ are square integrable in each compact subinterval of $(a, b)$.

Parenthetically, we note that the telegraphist equation

$$
q u_{t t}+r u_{t}=\left(p u_{x}\right)_{x}-s u
$$

can be brought into the form (1.1); here we suppose that $p>0, q>0, r \geqq 0$, and $s>0$ for all $x \in(a, b)$. In fact, setting $u=\eta^{1}, u_{x}=\eta^{2}$, and $u_{t}=\eta^{3}$, the solution of (1.3) is readily seen to satisfy (1.1) with

(2) Condition (D) could be replaced by ( $\left.\mathrm{D}^{\prime}\right) B+B^{*}+A_{x} \leqq 2 \gamma E, a<x<b$, for some real constant $\gamma$. However the transformation $y \rightarrow y^{\prime} e^{\gamma_{t}}$ brings $\left(D^{\prime}\right)$ back into the form (D) so that we may use (D) without loss of generality.

(3) Hereafter we use the expression "absolutely continuous" to mean that the function is absolutely continuous on each compact subinterval of $(a, b)$. 


$$
E=\left(\begin{array}{lll}
s & 0 & 0 \\
0 & p & 0 \\
0 & 0 & q
\end{array}\right), \quad A=\left(\begin{array}{lll}
0 & 0 & 0 \\
0 & 0 & p \\
0 & p & 0
\end{array}\right), \quad B=\left(\begin{array}{rlr}
0 & 0 & s \\
0 & 0 & -p_{x} \\
-s & 0 & -r
\end{array}\right)
$$

In this case

$$
B+B^{*}+A_{x}=\left(\begin{array}{rrr}
0 & 0 & 0 \\
0 & 0 & 0 \\
0 & 0 & -2 r
\end{array}\right) \leqq \Theta .
$$

Conversely, suppose $y=\left(\eta^{1}, \eta^{2}, \eta^{3}\right)$ is a solution of the system (1.1) with coefficient matrices (1.4) and suppose that the initial data has been chosen so that $\eta^{2}(x, 0)=\eta^{1}(x, 0)_{x}$. Since $\eta_{t}^{2}=\eta_{x}^{3}=\eta_{t x}^{1}$, it follows that $\eta^{2}(x, t)=\eta^{1}(x, t)_{x}$ for all $t>0$ and hence that such a solution of the system satisfies (1.3) (the smoothness properties of solutions to the differential system which are required for this argument are established at the close of $\$ 7$ ).

The energy integral for system (1.1) is

$$
\text { Energy }=\frac{1}{2} \int_{a}^{b}(E y, y) d x .
$$

Since we shall restrict our considerations to solutions of (1.1) for which the energy is nonincreasing in time, a natural setting for the problem is the hilbert space $I I=L_{2}(a, b ; E)$ with

(1.7) inner product $\langle y, z\rangle=\int_{a}^{b}(E y, z) d x$ and norm $\|y\|=[\langle x, y\rangle]^{1 / 2}$.

From (1.1) we obtain, at least formally, the relation

$$
\langle y, y\rangle_{t}=\left[(\Lambda y, y)^{b}-(\Lambda y, y)^{a}\right]+\int_{a}^{b}\left(\left(B+B^{*}+A_{x}\right) y, y\right) d x .
$$

The integral on the right is the rate at which energy enters the system from interior sources, whereas the expression $\left[(A y, y)^{b}-(A y, y)^{a}\right]$ is the rate at which energy enters the system through the boundary. Thus the condition that there be no interior energy sources is precisely (D) and, with (D) assumed, the energy will be nonincreasing in time if we impose boundary conditions on $y$ such that

$$
(A y, y)^{b}-(A y, y)^{a} \leqq 0 ;
$$

boundary conditions of this kind will be called dissipative.

The Cauchy problem for the system (1.1) can now be formulated in terms of semi-groups of operators. We require of the operator

$$
L y=E^{-1}\left[(A y)_{x}+B y\right] \text {, }
$$


with a domain $\mathfrak{D}(L)$ suitably restricted by dissipative boundary conditions, that it generate a strongly continuous semi-group of linear bounded operators, say $[S(t) ; t \geqq 0]$. In this case the initial value is assumed in the mean square sense, that is,

$$
\lim _{t \rightarrow 0+} S(t) y_{0}=y_{0}, \quad y_{0} \in H,
$$

in norm; and the differential equation is satisfied in the sense that

$$
d S(t) y_{0} / d t=L\left[S(t) y_{0}\right], \quad y_{0} \in \mathfrak{D}(L), t>0,
$$

the derivative being taken in the norm topology of $H$. Employing these results it is possible to go somewhat beyond the theory of semi-groups and show for each $y_{0} \in \mathfrak{D}\left(L^{2}\right)$ that there is a representation of $y(\cdot, t)=S(t) y_{0}$ which satisfies (1.1) even in the classical sense (see the end of $\$ 7$ ). It follows from the relation (1.8) that condition (D) together with the requirement that $L$ have dissipative boundary conditions forces the operators $S(t)$ to be contraction operators, that is, operators of norm less than or equal to one.

With this semi-group setting the Cauchy problem for (1.1) can be given an exceedingly precise expression. To this end we let

$$
D=E-\left(B+B^{*}+A_{x}\right)
$$

and denote by $L_{2}(a, b ; D)$ the class of all vector-valued measurable functions for which

$$
\int_{a}^{b}(D y, y) d x<\infty
$$

We further define

$$
\begin{aligned}
L_{1} y= & E^{-1}\left[(A y)_{x}+B y\right], \\
\mathfrak{D}\left(L_{1}\right)= & {\left[y ; y \in L_{2}(a, b ; D), A y\right. \text { absolutely continuous, and }} \\
& \left.E^{-1}\left[(A y)_{x}+B y\right] \in L_{2}(a, b ; E)\right] .
\end{aligned}
$$

If, in the definition of $\mathfrak{D}\left(L_{1}\right)$, the condition $y \in L_{2}(a, b ; D)$ were replaced by $y \in L_{2}(a, b ; E)$ then it would be clear that the domain contains all vector functions in $\mathfrak{D}(L)$, assuming $L$ to be defined in a reasonable way $\left.{ }^{4}\right)$. However, for $y^{\prime}$ in the so extended $\mathfrak{D}\left(L_{1}\right)$ the limits

$$
(A y, y)^{a}=\lim _{x \rightarrow a+}(A y, y)^{x} \text { and }(A y, y)^{b}=\lim _{x \rightarrow b-}(A y, y)^{x}
$$

exist if and only if $y \in L_{2}(a, b ; D)$; and, since we insist on imposing dissipative boundary conditions, there is no loss in generality in requiring $L$ to be a re-

(4) It can be shown that $L_{1}$ is a closed operator both for $\mathfrak{D}\left(L_{1}\right)$ defined as in (1.14) and for the modified $\mathfrak{D}\left(L_{1}\right)$ defined as in (5.7); see Lemma 5.2. 
striction of $L_{1}$, defined as in (1.14). The problem, then, is to find all possible dissipative restrictions of $L_{1}$ which generate strongly continuous semigroups of operators $\left({ }^{5}\right)$.

According to the Hille-Yosida theorem (see E. Hille and R. S. Phillips [7, Theorem 12.3.1]) a closed linear operator $L$ with dense domain generates a strongly-continuous semi-group of linear bounded contraction operators if and only if the resolvent of $L$, namely $R(\lambda ; L)$, satisfies the condition

$$
\lambda\|R(\lambda ; L)\| \leqq 1
$$

for all sufficiently large real $\lambda$. In order to verify (1.15) we shall construct an explicit representation for the resolvent out of the solutions of the homogeneous system

$$
\lambda y-E^{-1}\left[(A y)_{x}+B y\right]=0,
$$$$
a<x<b,
$$

and its formal adjoint

$$
\lambda z-E^{-1}\left[-(A z)_{x}+\left(B^{*}+A_{x}\right) z\right]=0,
$$$$
a<x<b,
$$

both of which are studied in $\S 3$. The domain of a dissipative restriction of $L_{1}$ can then be defined by means of $r$ suitably chosen linearly independent pairs of solutions to $(1.17)$, say $\left[z_{a, i}, z_{b, i}\right]$, as

(1.18) $\mathfrak{D}(L)=\left[y ; y \in \mathfrak{D}\left(L_{1}\right),\left(A y, z_{b, i}\right)^{b}-\left(A y, z_{a, i}\right)^{a}=0\right.$ for $\left.i=1,2, \cdots, r\right]$; this is the end result of $\S 4$.

It is desirable to free the definition of $\mathfrak{D}(L)$ of its dependence on $\lambda$. To this end we introduce the dual of $L_{1}$, namely

$$
M_{1} z=E^{-1}\left[-(A z)_{x}+\left(B^{*}+A_{x}\right) z\right]
$$

$$
\begin{aligned}
\mathfrak{D}\left(M_{1}\right)= & {\left[z ; z \in L_{2}(a, b ; D), A z\right. \text { absolutely continuous, and }} \\
& \left.E^{-1}\left[-(A z)_{x}+\left(B^{*}+A_{x}\right) z\right] \in L_{2}(a, b ; E)\right],
\end{aligned}
$$

which is again dissipative since

$$
\left(B^{*}+A_{x}\right)+\left(B^{*}+A_{x}\right)^{*}+(-A)_{x}=B+B^{*}+A_{x} \leqq \Theta .
$$

Following K. Kodaira [8], we then consider $\mathfrak{D}\left(L_{1}\right)$ modulo

$$
\mathfrak{D}\left(L_{b}\right)=\left[y ; y \in \mathfrak{D}\left(L_{1}\right),(A y, z)^{b}=0 \text { for all } z \in \mathfrak{D}\left(M_{1}\right)\right] \text {. }
$$

This quotient space is finite dimensional and preserves all of the relevant behavior of $y \in \mathfrak{D}\left(L_{1}\right)$ at the boundary $b$; in other words $\left(A y_{1}, y_{2}\right)^{b}$ depends only on the cosets $Y_{b, 1}, Y_{b, 2}$ containing $y_{1}$ and $y_{2}$, respectively. We use this limit to define $\left(A Y_{b, 1}, Y_{b, 2}\right)$. Treating $\mathfrak{D}\left(M_{1}\right)$ in the same fashion, we find that the quotient spaces $\mathfrak{D}\left(L_{1}\right) / \mathfrak{D}\left(L_{b}\right)$ and $\mathfrak{D}\left(M_{1}\right) / \mathfrak{D}\left(M_{b}\right)$ are of the same dimen-

(5) It should be noted that we actually do not obtain the most general semi-group solution with noncreasing energy since we require that both terms in the right member of (1.8) be nonpositive whereas the energy is nonincreasing if merely the sum is nonpositive. 
sion, having bases $U_{b, 1}, U_{b, 2}, \cdots, U_{b, d_{b}}$ and $V_{b, 1}, V_{b, 2}, \cdots, V_{b, d_{b}}$, respectively, such that for $Y=\sum \gamma_{i} U_{b, i}$ and $Z=\sum \delta_{i} V_{b, i}$ we have

$$
\begin{aligned}
& \left(A Y_{b}, Y_{b}\right)=\sum \gamma_{i} \bar{\gamma}_{j} \mu_{i j}, \\
& \left(A Y_{b}, Z_{b}\right)=\sum \gamma_{i} \bar{\delta}_{i}, \\
& \left(A Z_{b}, Z_{b}\right)=\sum \delta_{i} \bar{\delta}_{j} \nu_{i j},
\end{aligned}
$$

where $\left(\mu_{i j}\right)=\left(\nu_{i j}\right)^{-1}$ is nonsingular and hermitian with $n_{b}$ negative and $p_{b}$ positive eigenvalues. Analogous results hold at the $a$ end. Now let $\mathfrak{N}_{a, b}$ be a linear subspace of $\mathfrak{D}\left(L_{1}\right) / \mathfrak{D}\left(L_{a}\right) \times \mathfrak{D}\left(L_{1}\right) / \mathfrak{D}\left(L_{b}\right)$ of dimension $p_{a}+n_{b}$ such that $-\left(A Y_{a}, Y_{a}\right)+\left(A Y_{b}, Y_{b}\right) \leqq 0$ for all $\left[Y_{a}, Y_{b}\right] \in \mathfrak{N}_{a, b}$ and let $\mathfrak{P}_{a, b}$ be the $A_{a, b}$ orthogonal complement of $\mathfrak{R}_{a, b}$ in $\mathfrak{D}\left(M_{1}\right) / \mathfrak{D}\left(M_{a}\right) \times \mathfrak{D}\left(M_{1}\right) / \mathfrak{D}\left(M_{b}\right)$, that is,

$$
\mathfrak{B}_{a, b}=\left[\left[Z_{a}, Z_{b}\right] ;-\left(A Y_{a}, Z_{a}\right)+\left(A Y_{b}, Z_{b}\right)=0 \text { for all }\left[Y_{a}, Y_{b}\right] \subset \mathfrak{R}_{a, b}\right] \text {. }
$$

Then $\Re_{a, b}$ will be of dimension $n_{a}+p_{b}$ and $-\left(A Z_{a}, Z_{a}\right)+\left(A Z_{b}, Z_{b}\right) \geqq 0$ for all $\left[Z_{a}, Z_{b}\right] \in \mathfrak{B}_{a, b}$. The most general dissipative restrictions of $L_{1}$ and $M_{1}$ which generate semi-groups are of the form

$$
\begin{aligned}
\mathfrak{D}(L) & =\left[y ; y \in \mathfrak{D}\left(L_{1}\right),[y, y] \rightarrow \mathfrak{N}_{a, b}\right], \\
\mathfrak{D}(M) & =\left[z ; z \in \mathfrak{D}\left(M_{1}\right),[z, z] \rightarrow \mathfrak{P}_{a, b}\right] ;
\end{aligned}
$$

and, conversely, any such $L$ and $M$ are dissipative generators. Incidentally, $L$ and $M$ as so defined are adjoints to each other. We note that $\mathfrak{D}(L)$ can be defined equivalently by a representative set of functions $\left\{\left[z_{a, i}, z_{b, i}\right]\right.$; $\left.i=1,2, \cdots, n_{a}+p_{b}\right\} \subset \mathfrak{D}\left(M_{1}\right) \times \mathfrak{D}\left(M_{1}\right)$ which map into cosets spanning $\mathfrak{P}_{a, b}$, in which case

$$
\begin{aligned}
\mathfrak{D}(L)=\left[y ; y \in \mathfrak{D}\left(L_{1}\right),-\left(A y, z_{a, i}\right)^{a}+\left(A y, a_{b, i}\right)^{b}\right. & =0 \\
& \text { for } \left.i=1,2, \cdots, n_{a}+p_{b}\right] .
\end{aligned}
$$

This solves the problem which we set above.

Thus far the development still does not furnish us with a solution to the simple vibrating string with elastic end conditions (i.e. $u+\beta u_{x}=0$ at $x=b$ ). However, by making use of these quotient spaces we can also define suitable couplings at each end of the differential system to simple finite degree of freedom systems so as to obtain the analogues of the elastic end conditions; this is done in $\$ 7$.

A system will be called conservative if energy neither enters nor leaves. Thus the operator $L$ will be conservative if equality holds in (D) and the boundary conditions are such that equality holds in (1.9) for all $y \in \mathfrak{D}(L)$; similar remarks apply to operators for the coupled systems. It follows from (1.8) that the semi-group generated by a conservative operator will consist of isometries, that is, norm preserving operators. Of particular interest is the case where these operators are actually unitary operators for then $L$ generates a group and the process is reversible in time. Necessary and sufficient condi- 
tions on the differential system for $L$ to generate a group of unitary operators are obtained in $\S 8$. It is interesting to note that the solution to the conservative wave equation (1.3) with conservative boundary conditions is always reversible in the above sense.

Finally in $\S 9$ we extend the previous development to coupled systems for which there is a direct coupling between the interior of the differential system and the finite degree of freedom end systems. This is accomplished by means of a perturbation technique (see R. S. Phillips [12]).

2. Transformation theory. It will be convenient to bring the differential system (1.1) into a normal form and to this end we now develop a suitable transformation theory. We note first of all that corresponding coefficient matrices of $L_{1}$ and $M_{1}$ satisfy the same smoothness assumptions and that condition (D) makes both systems dissipative.

As we have previously stated, the natural setting for our problem is the hilbert space $L_{2}(a, b ; E)$. We now consider a unitary mapping: $y=V y^{\prime}$ of a second hilbert space $L_{2}\left(a, b ; E^{\prime}\right)$ onto $L_{2}(a, b ; E)$, the map $V$ being of the form

$$
y(x)=V(x)\left[y^{\prime}(x)\right], \quad a<x<b,
$$

where $V(x)$ is a $k \times k$ nonsingular matrix for each $x \in(a, b)$ with elements which are absolutely continuous and possess square integrable derivatives in each compact subinterval of $(a, b)$. Since $V$ is in particular an isometry, we must have

$$
\int_{a}^{b}(E y, y) d x=\int_{a}^{b}\left(E^{\prime} y^{\prime}, y^{\prime}\right) d x
$$

for all $y^{\prime} \in L_{2}\left(a, b ; E^{\prime}\right)$ and this together with the continuity of the matrices involved implies

$$
E^{\prime}(x)=[V(x)]^{*} E(x) V(x), \quad a<x<b .
$$

The nonsingular character of the matrices $V(x)$ suffices to show that the mapping $V$ is indeed one-to-one and onto. We note that $E^{\prime}(x)$ as defined in (2.2) is again hermitian, positive definite, and absolutely continuous with derivatives which are square integrable in each compact subset of $(a, b)$.

Suppose now that $L$ and $M$ are restrictions of $L_{1}$ and $M_{1}$, respectively, with domains

$$
\begin{aligned}
\mathfrak{D}(L) & =\left[y ; y \in \mathfrak{D}\left(L_{1}\right),\left(A y, z_{a, i}\right)^{a}-\left(A y, z_{b, i}\right)^{b}=0, i=1, \cdots, q\right], \\
\mathfrak{D}(M) & =\left[z ; z \in \mathfrak{D}\left(M_{1}\right),\left(A z, y_{a, i}\right)^{a}-\left(A z, y_{b, i}\right)^{b}=0, i=1, \cdots, q\right],
\end{aligned}
$$

where the $\left[y_{a, i}, y_{b, i}\right]$ and the $\left[z_{a, i}, z_{b, i}\right]$ are function pairs in $\mathfrak{D}\left(L_{1}\right) \times \mathfrak{D}\left(L_{1}\right)$ and $\mathfrak{D}\left(M_{1}\right) \times \mathfrak{D}\left(M_{1}\right)$ respectively. Then the transformed operators are given by 


$$
\begin{aligned}
L^{\prime} & =V^{-1} L V, & \mathfrak{D}\left(L^{\prime}\right) & =V^{-1}[\mathfrak{D}(L)], \\
M^{\prime} & =V^{-1} M V, & \mathfrak{D}\left(M^{\prime}\right) & =V^{-1}[\mathfrak{D}(M)] .
\end{aligned}
$$

A straightforward calculation shows that

$$
\begin{aligned}
L^{\prime} y^{\prime} & =E^{\prime-1}\left[\left(A^{\prime} y^{\prime}\right)_{x}+B^{\prime} y^{\prime}\right], \\
M^{\prime} y^{\prime} & =E^{\prime-1}\left[-\left(A^{\prime} z^{\prime}\right)_{x}+\left(B^{\prime *}+A_{x}^{\prime}\right) z^{\prime}\right],
\end{aligned}
$$

where

$$
\begin{aligned}
& A^{\prime}(x)=V(x)^{*} A(x) V(x) \text { and } \\
& B^{\prime}(x)=V(x)^{*} B(x) V(x)-\left[V(x)^{*}\right]_{x} A(x) V(x) .
\end{aligned}
$$

We see that $A^{\prime}(x)$ and $B^{\prime}(x)$ have the same smoothness properties as $A(x)$ and $B(x)$, respectively. Further

$$
B^{\prime}+B^{\prime *}+A_{x}^{\prime}=V^{*}\left(B+B^{*}+A_{x}\right) V
$$

so that the transformed system satisfies the condition (D) if and only if this is the case for the original system and even the condition $B+B^{*}+A_{x}=\Theta$ remains invariant.

As to the domains of the transformed operators, we note that $A y$ and $A^{\prime} y^{\prime}$ are absolutely continuous together and that

$$
\int_{a}^{b}(D y, y) d x=\int_{a}^{b}\left(D^{\prime} y^{\prime}, y^{\prime}\right) d x
$$

As a consequence

$$
\begin{aligned}
\mathfrak{D}\left(L_{1}^{\prime}\right) \equiv & V^{-1}\left[\mathfrak{D}\left(L_{1}\right)\right]=\left[y^{\prime} ; y^{\prime} \in L_{2}\left(a, b ; D^{\prime}\right), A^{\prime} y^{\prime}\right. \text { absolutely continuous, } \\
& \text { and } \left.E^{\prime-1}\left[\left(A^{\prime} y^{\prime}\right)_{x}+B^{\prime} y^{\prime}\right] \in L_{2}\left(a, b ; E^{\prime}\right)\right] .
\end{aligned}
$$

Similarly $\mathfrak{D}\left(M_{1}^{\prime}\right)$ has the same form as $\mathfrak{D}\left(M_{1}\right)$. Finally for $y=V y^{\prime}$ and $z=V z^{\prime}$ we have

$$
(A y, z)=\left(A V y^{\prime}, V z^{\prime}\right)=\left(A^{\prime} y^{\prime}, z^{\prime}\right), \quad a<x<b,
$$

and this shows that $\mathfrak{D}\left(L^{\prime}\right)$ and $\mathfrak{D}(L)$ also have the same form if we replace the restricting functions $\left[z_{a, i}, z_{b, i}\right]$ in $\mathfrak{D}(L)$ by their transforms. Likewise $\mathfrak{D}\left(M^{\prime}\right)$ has the same form as $\mathfrak{D}(M)$.

The adjoint relation between $L_{1}$ and $M_{1}$ can now be made somewhat more precise. We note that if $A y$ and $A z$ are absolutely continuous then so are $A^{\prime} y^{\prime}$ and $A^{\prime} z^{\prime}$ and we have for almost all $x \in(a, b)$

$$
\begin{aligned}
(A y, z)_{x}+\left(A_{x} y, z\right)-\left((A y)_{x}, z\right)-\left(y,(A z)_{x}\right) & \\
= & \left(A^{\prime} y^{\prime}, z^{\prime}\right)_{x}+\left(A_{x}^{\prime} y^{\prime}, z^{\prime}\right)-\left(\left(A^{\prime} y^{\prime}\right)_{x}, z^{\prime}\right)-\left(y^{\prime},\left(A^{\prime} z^{\prime}\right)_{x}\right) .
\end{aligned}
$$

As we shall see (Equation (2.19)), when the transformed system is in normal form the right hand side of (2.11) vanishes so that 


$$
(A y, z)_{x}=\left((A y)_{x}, z\right)+\left(y,(A z)_{x}\right)-\left(A_{x} y, z\right)
$$

almost everywhere. From this we see that $L_{1}$ and $M_{1}$ are essentially adjoints; in fact, for $y \in \mathfrak{D}\left(L_{1}\right)$ and $z \in \mathfrak{D}\left(M_{1}\right)$ we obtain

$$
\left\langle L_{1} y, z\right\rangle-\left\langle y, M_{1} z\right\rangle=(A y, z)^{b}-(A y, z)^{a} .
$$

By applying two successive transformations of the above type we bring the operator $L_{1}$ into a normal form. First we write

$$
V(x)=[E(x)]^{-1 / 2} \equiv(2 \pi i)^{-1} \int_{\Gamma(x)} \lambda^{-1 / 2} R(\lambda ; E(x)) d \lambda ;
$$

here $\Gamma(x)$ is a simple closed rectifiable path lying in the right half-plane and containing in its interior the spectrum of $E(x)$, namely $\sigma[E(x)]$, and the principal determination of $\lambda^{-1 / 2}$ is used. Now for a fixed $x_{0} \in(a, b)$, the path $\Gamma\left(x_{0}\right)$ will contain $\sigma[E(x)]$ for $\left|x-x_{0}\right|<\delta\left(x_{0}\right)$ and since the elements of $R(\lambda ; E(x))$ are absolutely continuous with square integrable derivatives for $\lambda \in \Gamma\left(x_{0}\right)$ and $\left|x-x_{0}\right|<\delta\left(x_{0}\right)$, it follows from the above representation that this will also be true for $[E(x)]^{-1 / 2}$. Hence the so defined $V(x)$ is a suitable transforming matrix function. Finally with $V(x)=[E(x)]^{-1 / 2}$ we see that $E^{\prime}(x) \equiv I$.

The second transformation which we use to bring the operator $L_{1}$ into normal form is a unitary transformation of $L_{2}(a, b ; I)$ onto itself, which separates out the null eigenspaces of $A(x), a<x<b$, from the non-null eigenspaces. We put the formal argument in the form of a lemma.

LemMa 2.1. Let $A(x), a<x<b$, be hermitian, of constant rank $r$, and suppose the elements of $A(x)$ are absolutely continuous with square integrable derivatives on each compact subinterval of $(a, b)$. Then there exists a family of unitary matrices $[U(x) ; a<x<b]$, with elements absolutely continuous and having square integrable derivatives in each compact subinterval of $(a, b)$, such that

$$
U(x)^{*} A(x) U(x)=\left(\begin{array}{cc}
0 & 0 \\
0 & A_{11}^{\prime}(x)
\end{array}\right),
$$

where the upper left element is the zero $(k-r) \times(k-r)$ matrix and the $A_{1}^{\prime 1}(x)$ is a nonsingular $r \times r$ matrix for each $x \in(a, b)$.

Remark. In general it is not possible to diagonalize $A(x)$ by a matrixvalued function with the required smoothness properties.

Proof. It is clear that the columns of $U(x)$ consist of mutually orthogonal vectors, the first $k-r$ of which span the null eigenspace of $A(x)$ and the last $r$ of which span the non-null eigenspace of $A(x)$. The only other condition on $U(x)$ is that its elements be sufficiently smooth.

Now $A(x)$ is continuous on $(a, b)$ and of constant rank. Consequently for each compact subinterval $\left[a^{\prime}, b^{\prime}\right]$ of $(a, b)$, we can find a circle $\Gamma^{\prime}$ with the 
origin as center which excludes the nonzero part of the spectrum of $A(x)$ for all $x \in\left[a^{\prime}, b^{\prime}\right]$. In this case

$$
P(x)=(2 \pi i)^{-1} \int_{\Gamma^{\prime}} R(\lambda ; A(x)) d \lambda
$$

projects the space onto the $(k-r)$-dimensional null eigenspace of $A(x)$, $a^{\prime}<x<b^{\prime}$. As before the elements of $P(x)$ will be absolutely continuous with square integrable derivatives in $\left[a^{\prime}, b^{\prime}\right]$. Hence there is a finite subdivision $x_{0}=a^{\prime}<x_{1}<\cdots<x_{q}=b^{\prime}$ such that in each subinterval

$$
\max \left[\left|P\left(x_{i}\right)-P(x)\right| ; x_{i} \leqq x \leqq x_{i+1}\right]<1 / 2 .
$$

Suppose next that $U(x)$ has been defined for $a^{\prime} \leqq x \leqq x_{i}$ and let $u_{j}\left(x_{i}\right)$ be the $j$ th column vector in $U\left(x_{i}\right)$. Then for $j \leqq k-r$, the vector $u_{j}\left(x_{i}\right)$ will lie in the range of $P\left(x_{i}\right)$. Setting $v_{j}(x)=P(x) u_{j}\left(x_{i}\right), j \leqq k-r$, we see that

$$
\begin{aligned}
&\left|\sum_{j=1}^{k-r} \gamma_{j} v_{j}(x)\right| \geqq\left|\sum_{j=1}^{k-r} \gamma_{j} u_{j}\left(x_{i}\right)\right|-\left|\sum_{j=1}^{k-r} \gamma_{j} v_{j}(x)-\sum_{j=1}^{k-r} \gamma_{j} u_{j}\left(x_{i}\right)\right| \\
&=\left|\sum_{j=1}^{k-r} \gamma_{j} u_{j}\left(x_{i}\right)\right|-\left|\left[P(x)-P\left(x_{i}\right)\right] \sum_{j=1}^{k-r} \gamma_{j} u_{j}\left(x_{i}\right)\right| \\
& \geqq \frac{1}{2}\left|\sum_{j=1}^{k-r} \gamma_{j} u_{j}\left(x_{i}\right)\right|, \\
& x_{i} \leqq x \leqq x_{i+1} .
\end{aligned}
$$

Since the $\left[u_{j}\left(x_{i}\right) ; j \leqq k-r\right]$ are linearly independent, the same is true of the $\left[v_{j}(x) ; j \leqq k-r\right]$ for each $x, x_{i} \leqq x \leqq x_{i+1}$. If we now make use of the orthogonalization process, we obtain the orthogonal set of vectors $\left[u_{j}(x) ; j \leqq k-r\right]$, having the properties (i) $u_{j}\left(x_{i}\right)=v_{j}\left(x_{i}\right)$, and (ii) the $u_{j}(x)$ are absolutely continuous with square integrable derivatives in $\left[x_{i}, x_{i+1}\right]$. Proceeding in the same fashion with respect to $I-P(x)$, which projects onto the non-null eigenspace of $A(x)$, we fill out the rest of the columns of $U(x)$ over the range $x_{i} \leqq x \leqq x_{i+1}$. Thus a finite number of steps suffices to define $U(x)$ over the interval $\left[a^{\prime}, b^{\prime}\right]$ and it is clear that we can proceed in a denumerable number of steps to define $U(x)$ over all of $(a, b)$ to have the desired properties. This concludes the proof.

Starting with a system with $E(x) \equiv I$, we now apply the transformation given by the lemma, namely

$$
V: y(x)=U(x) y^{\prime}(x) \text {. }
$$

According to our general theory, $E^{\prime}(x)=U(x) * I U(x)=I$ and by the above construction

$$
A^{\prime}(x)=\left(\begin{array}{cc}
0 & 0 \\
0 & A_{11}^{\prime}(x)
\end{array}\right), \quad B^{\prime}(x)=\left(\begin{array}{cc}
B_{00}^{\prime}(x) & B_{01}^{\prime}(x) \\
B_{10}^{\prime}(x) & B_{11}^{\prime}(x)
\end{array}\right),
$$


where the upper left block is $(k-r) \times(k-r)$ and the lower right block is $r \times r$, $A_{11}^{\prime}(x)$ being hermetian and nonsingular. This is the desired normal form. We note that the same series of transformations also brings the adjoint operator into normal form. It is convenient when dealing with such an operator in normal form to introduce the notation

$$
y^{0}=\left(\eta^{1}, \cdots, \eta^{k-r}\right) \text { and } y^{1}=\left(\eta^{k-r+1}, \cdots, \eta^{k}\right) ;
$$

we then have $\left(A^{\prime} y^{\prime}, z^{\prime}\right)=\left(A_{11}^{\prime} y^{\prime 1}, z^{\prime 1}\right)$. Moreover if $A^{\prime} y^{\prime}$ is absolutely continuous, then so is $A_{11}^{\prime} y^{\prime 1}$, and since $A_{11}^{\prime}$ is nonsingular and absolutely continuous, $y^{\prime 1}$ will also be absolutely continuous. It now follows for $A^{\prime} y^{\prime}$ and $A^{\prime} z^{\prime}$ both absolutely continuous in $x$, that almost everywhere

$$
\begin{aligned}
\left(A^{\prime} y^{\prime}, z^{\prime}\right)_{x} & =\left(A_{11}^{\prime} y^{\prime 1}, z^{\prime 1}\right)_{x} \\
& =\left(\left(A_{11}^{\prime} y^{\prime 1}\right)_{x}, z^{\prime 1}\right)+\left(y^{\prime 1},\left(A_{11}^{\prime} z^{\prime}\right)_{x}\right)-\left(\left(A_{11}^{\prime}\right)_{x} y^{\prime 1}, z^{\prime 1}\right) \\
& =\left(\left(A^{\prime} y^{\prime}\right)_{x}, z^{\prime}\right)+\left(y^{\prime},\left(A^{\prime} z^{\prime}\right)_{x}\right)-\left(A_{x}^{\prime} y^{\prime} z^{\prime}\right),
\end{aligned}
$$

which is the relation used in deriving (2.12).

We shall also have occasion to consider the unitary transformation resulting from a change in the independent variable. Suppose

$$
\xi(x)=\int_{c}^{x}[\rho(\sigma)]^{2} d \sigma, \quad \xi(a)=\alpha \quad \text { and } \quad \xi(b)=\beta,
$$

where $\rho(x)$ is absolutely continuous and greater than zero on $(a, b)$. In this case the inverse function, namely $x(\xi)$, is well defined and twice continuously differentiable. We set $E^{\prime}(\xi)=E[x(\xi)]$ and $\rho^{\prime}(\xi)=\rho[x(\xi)]$. Since we wish the map to be unitary, we define

$$
V: y(x) \in L_{2}(a, b ; E) \rightarrow y^{\prime}(\xi)=\left[\rho^{\prime}(\xi)\right]^{-1} y[x(\xi)] \in L_{2}\left(\alpha, \beta ; E^{\prime}\right) .
$$

A straightforward calculation shows that $L^{\prime}=V L V^{-1}$ and $M^{\prime}=V M V^{-1}$ are of the form

$$
\begin{aligned}
L^{\prime} y^{\prime} & =E^{\prime-1}\left[\left(A^{\prime} y^{\prime}\right) \xi+B^{\prime} y^{\prime}\right], \\
M^{\prime} z^{\prime} & =E^{\prime-1}\left[-\left(A^{\prime} z^{\prime}\right) \xi+\left(B^{\prime *}+A_{\xi}^{\prime}\right) z^{\prime}\right]
\end{aligned}
$$

where

$$
\begin{aligned}
& A^{\prime}(\xi)=\left[\rho^{\prime}(\xi)\right]^{2} A[x(\xi)], \\
& B^{\prime}(\xi)=B[x(\xi)]-\frac{1}{2}\left\{d\left[\rho^{\prime}(\xi)\right]^{2} / d \xi\right\} A[x(\xi)] .
\end{aligned}
$$

Again it is clear that the smoothness properties of the matrices are preserved, that

$$
B^{\prime}+B^{\prime *}+A_{\xi}^{\prime}=B[x(\xi)]+B[x(\xi)]^{*}+A_{x}[x(\xi)],
$$


and that $(A y, z)^{x(\xi)}=\left(A^{\prime} y^{\prime}, z^{\prime}\right)^{\xi}$. As before, $\mathfrak{D}\left(L^{\prime}\right)$ has the same form as $\mathfrak{D}(L)$; however, we omit the details.

From this point on we shall assume that our system is in normal form.

3. Solutions of the homogeneous equations. We proceed to solve the ordinary differential equation systems

$$
\lambda y-(A y)_{x}-B y=\theta,
$$

$$
a<x<b,
$$

and

$$
\lambda z+(A z)_{x}-\left(B^{*}+A_{x}\right) z=\theta,
$$$$
a<x<b,
$$

for $\lambda>0$; here $A$ and $B$ satisfy the assumptions stated in $\S 1$ and $A$ is given in normal form $\left(^{6}\right)$.

Thus $A$ and $B$ split into blocks as indicated in (2.17), the $r \times r$ matrix $A_{11}(x)$ being hermitian and nonsingular for each $x \in(a, b)$. Since $A_{11}(x)$ is also assumed to be continuous on $(a, b)$, its spectrum varies continuously with $x$ and is consequently bounded away from zero on each compact subinterval of $(a, b)$. It follows that $A_{11}(x)$ (and hence $A(x)$ ) is of constant signature throughout $(a, b)$. We shall denote by $n$ the number of negative and by $p$ the number of positive eigenvalues for $A(x) ; n$ and $p$ are independent of $x$ and $n+p=r$.

We write the normalized (3.1) in the form

$$
\begin{aligned}
\lambda y^{0}-B_{00} y^{0}-B_{01} y^{1} & =\theta, \\
\lambda y^{1}-\left(A_{11} y^{1}\right)_{x}-B_{11} y^{1}-B_{10} y^{0} & =\theta,
\end{aligned}
$$

for almost all $x \in(a, b)$. Condition (D) implies that $B_{00}+B_{00}^{*} \leqq \Theta$.

Lemma 3.1. If $B_{00}+B_{00}^{*} \leqq \Theta$, then for $\lambda>0$

$$
R_{00}(\lambda)=\left(\lambda I-B_{00}\right)^{-1}
$$

exists and $\lambda\left|R_{00}(\lambda)\right| \leqq 1$.

Proof. Set $f^{0}=\lambda y^{0}-B_{00} y^{0}$. Then

$$
2 \lambda\left(y^{0}, y^{0}\right)-\left(\left(B_{00}+B_{00}^{*}\right) y^{0}, y^{0}\right)=\left(f^{0}, y^{0}\right)+\left(y^{0}, f^{0}\right),
$$

and making use of the fact that $B_{00}+B_{00}^{*} \leqq \Theta$, we see that $\lambda\left(y^{0}, y^{0}\right) \leqq\left|\left(f^{0}, y^{0}\right)\right|$ $\leqq\left|f^{0}\right|\left|y^{0}\right|$. Consequently $\lambda\left|y^{0}\right| \leqq\left|f^{0}\right|$ which shows that $\left(\lambda I-B_{00}\right)$ is one-toone and has an inverse of norm $\leqq 1 / \lambda$.

With the aid of the above lemma we can now solve the first equation in (3.3) for $y^{0}$ in the form

$$
y^{0}=R_{00}(\lambda) B_{01} y^{1}
$$

${ }^{(6)}$ If $A$ is in normal form, the restrictions on $A$ and $B$ can be somewhat relaxed to the extent that the elements of $\left(A_{11}\right)_{x}$ and $B_{11}$ need merely be integrable (not necessarily square integrable) on each compact subinterval of $(a, b)$. 
We note that for $\lambda>0$, the elements of the matrix $R_{00}(\lambda)$ are measurable and bounded (by $1 / \lambda$ ) in $x$. Substituting this for $y^{0}$ in the second equation in (3.3) we obtain

$$
\lambda y^{1}-\left(A_{11} y^{1}\right)_{x}-B_{11} y^{1}-B_{10} R_{00}(\lambda) B_{01} y^{1}=\theta .
$$

Since the elements of $B_{10}$ and $B_{01}$ are square integrable in each compact subinterval of $(a, b)$, we see that the elements of both $B_{11}$ and $B_{10} R_{00}(\lambda) B_{01}$ are integrable in each compact subinterval of $(a, b)$. Finally setting $u^{1}=A_{11} y^{1}$, the system (3.6) goes into the form

$$
u_{x}^{1}-C_{11} u^{1}=\theta \text {, almost everywhere on }(a, b),
$$

where the elements of $C_{11}$ are integrable on each compact subinterval of $(a, b)$. Appealing to the standard existence theorem for systems of ordinary differential equations (see, for instance, E. A. Coddington and N. Levinson [1, problem 1, p. 97]), we see that there exist $r$ absolutely continuous vector-valued solutions $\left\{u_{1}^{1}, u_{2}^{1}, \cdots, u_{r}^{1}\right\}$ which are linearly independent for each $x \in(a, b)$ and further each absolutely continuous solution of (3.7) is linearly dependent on these $r$ solutions. Since $A_{11}(x)$ is absolutely continuous and nonsingular, the vector-valued functions $y_{i}^{1}=A_{11}^{-1} u_{i}^{1}, i=1,2, \cdots, r$, will be absolutely continuous and together form a complete set of linearly independent solutions to (3.6). Moreover, setting $y_{i}^{0}=R_{00}(\lambda) B_{01} y_{i}^{1}$, the so-defined components of $y_{i}^{0}$ will be square integrable on each compact subinterval of $(a, b)$. The vectorvalued functions $y_{i}=\left(y_{i}^{0}, y_{i}^{1}\right)$ now form a complete set of linearly independent solutions to (3.3).

We summarize these results as follows:

THEOREM 3.1. There exist $r$ linearly independent vector-valued solutions of (3.1), $\left\{y_{i}(x)\right\}$ with $y_{i}$ square integrable on each compact subinterval of $(a, b)$ and with $A y_{i}$ absolutely continuous. Any solution of (3.1) with these properties can be represented as a linear combination of these $r$ functions. The components $\left\{y_{i}^{1} ; i=1, \cdots, r\right\}$ are linearly independent for each $x \in(a, b)$. A similar assertion holds for the solutions of (3.2).

Hereafter we consider only solutions of (3.1) (and (3.2)) such that $A y$ is absolutely continuous; for such solutions $y(x)$ will be square integrable on each compact subinterval of $(a, b)$.

THEOREM 3.2. If $y(x)$ is a nontrivial solution of (3.1), then $(A y, y)$ is an increasing function of $x$ on $(a, b)$. If, in addition, $z(x)$ is a solution of (3.2), then $(A z, z)$ is a decreasing function of $x$ and $(A y, z)$ is constant on $(a, b)$.

Proof. Combining (2.12) with $\boldsymbol{y}=\boldsymbol{z}$ and (3.1) we readily obtain

$$
(A y, y)_{x}=2 \lambda(y, y)-\left(\left(B+B^{*}+A\right) y, y\right)
$$

for almost all $x \in(a, b)$. According to the previous theorem $(y, y)>0$ for all 
$x \in(a, b)$ if $y$ is a nontrivial solution of (3.1) and this together with condition (D) shows that $(A y, y)$ is actually increasing in $x$. The corresponding result for $(A z, z)$ follows in a similar fashion. Finally combining (2.12) with (3.1) and (3.2) gives $(A y, z)_{x}=0$ almost everywhere so that $(A y, z)$ is independent of $x$ on $(a, b)$.

Corollary. If $y$ is a solution of $(3.1)$, then $(A y, y)^{b}\left[(A y, y)^{a}\right]$ exists if and only if $y \in L_{2}(c, b ; D)\left[y \in L_{2}(a, c ; D)\right]$, where $a<c<b$.

Proof. The $\lim _{x \rightarrow b-}(A y, y)^{x}$ will exist if and only if $(A y, y)_{x}$ is integrable on $(c, b)$. On the other hand each of the terms on the right side of (3.8) is nonnegative and hence each must be integrable if $(A y, y)_{x}$ is integrable. Consequently $(A y, y)^{b}$ exists if and only if $y \in L_{2}(c, b ; D)$. The assertion about $(A y, y)^{a}$ follows in a similar way.

We shall also require the following simple geometric result.

Lemma 3.2. Suppose $A$ is an $r \times r$ nonsingular hermitian matrix with $n$ negative and $p$ positive eigenvalues; $r=n+p$. Let $N$ be an $n$ dimensional linear subspace of the complex euclidean $r$-space such that $(A y, y)<0(\leqq 0)$ for all nonzero $y \in N$. Finally let $P$ denote the $A$-orthogonal complemenent of $N$, that is

$$
P=[z ;(A y, z)=0 \text { for all } y \in N] \text {. }
$$

Then $P$ is $p$ dimensional, $(A y, z)>0(\geqq 0)$ for all nonzero $z \in P$, and $N$ is again the $A$-orthogonal complement to $P$.

Proof. Since $A$ is nonsingular, the map of $N$ under $A$, namely $A N$, is again an $n$-dimensional subspace. The subspace $P$, being the ordinary complement of $A N$, is consequently $p=r-n$ dimensional. Suppose $z_{0} \in P, z_{0} \neq \theta$, and $\left(A z_{0}, z_{0}\right) \leqq 0(<0)$. If $z_{0}$ also belonged to $N$ then $(A N, P)=0$ implies that $\left(A z_{0}, z_{0}\right)=0$. However this is impossible since in the first case $\left(A z_{0}, z_{0}\right)<0$ for $z_{0} \in N$ and in the second case (i.e. where $(A y, y) \leqq 0$ for all $\left.y \in N\right)$ we are supposing that $\left(A z_{0}, z_{0}\right)<0$; thus in both cases $z_{0} \notin N$. Setting $N_{1}$ equal to the linear extension of $N \cup z_{0}$, we see that $N_{1}$ is $n+1$ dimensional. A generic element of $N_{1}$ can be written in the form $y_{1}=y+\alpha z_{0}, y \in N$, and in view of the fact that $\left(A y, z_{0}\right)=0$ we will have

$$
\begin{aligned}
\left(A y_{1}, y_{1}\right) & =(A y, y)+\alpha\left(A z_{0}, y\right)+\bar{\alpha}\left(A y, z_{0}\right)+|\alpha|^{2}\left(A z_{0}, z_{0}\right) \\
& =(A y, y)+|\alpha|^{2}\left(A z_{0}, z_{0}\right) \leqq 0 .
\end{aligned}
$$

However, this implies that $A$ has $n+1$ nonpositive eigenvalues, contrary to our hypothesis. Thus, no such nonzero $z_{0} \in P$ exists, which proves the first assertion of the lemma. Finally it is clear that $N$ is contained in the $A$-orthogonal complement to $P$ and, since this complement must be of dimension $n=r-p$, we see that $N$ coincides with it.

Suppose we think of the $y$ and $z$ spaces as distinct with a connecting inner product 


$$
\{y, z\}=\sum_{i, j=1}^{r} \eta_{i} \gamma_{i} \bar{\zeta}_{i}
$$

where the matrix $\Gamma=\left(\gamma_{i j}\right)$ is hermitian and nonsingular. This replaces the inner product $\left(A y, z^{\prime}\right)$ of the previous lemma and, in effect, replaces $z^{\prime}$ by $z=\Gamma^{-1} A z^{\prime}$. Accordingly $\left(A z^{\prime}, z^{\prime}\right)=\left(\Gamma A^{-1} \Gamma z, z\right)$ so that Lemma 3.2 translates into the

COROllary. Let $A$ be an $r \times r$ nonsingular hermitian matrix with $n$ negative and $p$ positive eigenvalues. Suppose we are given two $r$-dimensional complex euclidean spaces $Y$ and $Z$ connected by the inner product (3.9). Let $N$ be an $n$-dimensional subspace of $Y$ with $(A y, y)<0(\leqq 0)$ for all nonzero $y \in N$ and let $P$ be the $\Gamma$-orthogonal complement of $N$ in $Z$, that is, $P=[z ;\{y, z\}=0$ for all $y \in N]$. Then $P$ is p-dimensional and $\left(\Gamma A^{-1} \Gamma z, z\right)>0(\geqq 0)$ for all nonzero $z \in P$. Finally $N$ is again the $\Gamma$-orthogonal complement to $P$ in $Y$.

We come now to the principal result of this section.

THEOREM 3.3. Let $F_{b}\left[F_{a}\right]$ be the set of all solutions of $(3.1)$ with $(A y, y)^{b}$ $<\infty\left[(A y, y)^{a}>-\infty\right]$ or, equivalently, which belong to $L_{2}(c, b ; D)\left[L_{2}(a, c ; D)\right]$, $a<c<b$, and let $C_{b}\left[C_{a}\right]$ be the subset of $F_{b}\left[F_{a}\right]$ for which $(A y, y)^{b} \leqq 0\left[(A y, y)^{a}\right.$ $\geqq 0]$. Then $F_{b}$ is a linear subspace of dimension $l_{b} \geqq n\left[l_{a} \geqq p\right]$ and, in fact, even $C_{b}\left[C_{a}\right]$ contains an n-dimensional [ $p$-dimensional] linear subset.

Proof. We have already (Corollary to Theorem 3.2) established for solutions of the homogeneous system the equivalence between $(A y, y)^{b}<\infty$ and $y$ belonging to $L_{2}(c, b ; D)$. It follows from the latter condition that $F_{a}$ is a linear subspace. Let $\left\{y_{1}, y_{2}, \cdots, y_{r}\right\}$ form a basis for the solutions of (3.1) (Theorem 3.1) and set $y=\sum_{i=1}^{r} \gamma_{i} y_{i}$. Then

$$
(A y, y)^{x}=\sum_{i, j=1}^{r} \gamma_{i} \bar{\gamma}_{j}\left(A y_{i}, y_{j}\right)^{x}=\sum_{i, j=1}^{r} \gamma_{i} \bar{\gamma}_{j}\left(A_{11} y_{i}^{1}, y_{j}^{1}\right)^{x} .
$$

If we let $Y_{11}=\left(y_{1}^{1}, y_{2}^{1}, \cdots, y_{r}^{1}\right)$ denote the $r \times r$ matrix with $j$ th column vector $y_{j}^{1}$, then $Y_{11}$ will be nonsingular for each $x$ by Theorem 3.1. The matrix of the form in (3.10) is the nonsingular hermitian matrix $Y_{11}(x)^{*} A_{11}(x) Y_{11}(x)$, which again has $p$ positive and $n$ negative eigenvalues. Thus if we define

$$
C_{x}=\left[y ;(A y, y)^{x} \leqq 0\right],
$$

then in terms of the $\left(\gamma_{1}, \gamma_{2}, \cdots, \gamma_{r}\right)$ coordinates each $C_{x}$ is a closed cone containing certain $n$-dimensional linear subspaces. According to Theorem 3.2, we will have $C_{x_{1}} \supset C_{x_{2}}$ if $x_{1}>x_{2}$ and hence

$$
C_{b}=\bigcap_{x<b} C_{x}
$$

Now the $n$-dimensional linear subspaces are compact (in a suitable topology) 
and therefore $C_{b}$ contains at least one $n$-dimensional linear subspace and $a$ fortiori $l_{b} \geqq n$. The results for the $a$ end are proved in a similar way.

An analogous assertion holds for the solutions of the adjoint homogeneous equation (3.2) since the two equations are of the same type. However, since $A$ has been replaced by $-A$, there will be the following differences. The set $G_{b}\left[G_{a}\right]$ of solutions of $(3.2)$ with $(A z, z)^{b}>-\infty\left[(A z, z)^{a}<\infty\right]$ or, equivalently, which belong to $L_{a}(c, b ; D)\left[L_{2}(a, c ; D)\right]$ will be of dimension $m_{b} \geqq p\left[m_{a} \geqq n\right]$ and even the set for which $(A z, z)^{b} \geqq 0\left[(A z, z)^{a} \leqq 0\right]$ will contain a $p$-dimensional ( $n$-dimensional) linear subset.

We are now in a position to construct the Green's function for dissipative operators. This we proceed to do, leaving until later the proof that such an operator actually defines the resolvent for the most general dissipative restriction of $L_{1}$ which generates a semi-group.

We first consider solution pairs $\left[y_{o}, y_{b}\right]$ of (3.1) from which we choose an $r$-dimensional subspace $N_{a, b}$ such that

$$
-\left(A y_{a}, y_{a}\right)^{a}+\left(A y_{b}, y_{b}\right)^{b} \leqq 0
$$

for all $\left[y_{a}, y_{b}\right] \in N_{a, b}$. At least one such $r$-dimensional subspace $N_{a, b}$ exists since, according to Theorem 3.3, there is a p-dimensional subspace $N_{a}$ of $C_{a}$ and an $n$-dimensional subspace $N_{b}$ of $C_{b}$ whose product set $N_{a} \times N_{b}$ defines an $r$-dimensional subspace of the type $N_{a, b}$. It is clear from Theorem 3.2 that $(A y, y)^{b}>-\infty$ and $(A y, y)^{a}<\infty$ for any solution of (3.1) so that condition (3.12) requires that both $\left(A y_{b}, y_{b}\right)^{b}$ and $\left(A y_{a}, y_{a}\right)^{a}$ be finite; consequently $N_{a, b}$ is a linear subspace of $F_{a, b}=F_{a} \times F_{b}$.

We next define the $A_{a, b}$-orthogonal complement to $N_{a, b}$, namely the set of all solution pairs $\left[z_{a}, z_{b}\right]$ of (3.2) such that

$$
-\left(A y_{a}, z_{a}\right)^{a}-\left(A y_{b}, z_{b}\right)^{b}=0
$$

for all $\left[y_{a}, y_{b}\right] \in N_{a, b}$, and we denote this set by $P_{a, b}$. It follows from Theorem 3.2 that $(A y, z)^{x}$ is independent of $x$ for all solutions $y$ of (3.1) and $z$ of (3.2). As a consequence the condition (3.13) is equivalent with $-\left(A y_{a}, z_{a}\right)^{x_{1}}$ $+\left(A y_{b}, z_{b}\right)^{x_{2}}=0$ for some (and hence for all) $x_{1}, x_{2} \in(a, b)$.

Theorem 3.4. The set $P_{a, b}$ forms an $r$-dimensional linear subspace of solution pairs $\left[z_{a}, z_{b}\right]$ of (3.2) which is contained in $G_{a, b}=G_{a} \times G_{b}$ and has the property that

$$
-\left(A z_{a}, z_{a}\right)^{a}+\left(A z_{b}, z_{b}\right)^{b} \geqq 0
$$

for all $\left[z_{a}, z_{b}\right] \in P_{a, b}$. Moreover $N_{a, b}$ can be defined dually as the $A_{a, b}$-orthogonal complement of $P_{a, b}$.

Proof. We see by Theorem 3.2 that for $x_{1}, x_{2} \in(a, b)$

$$
-\left(A y_{a}, y_{a}\right)^{x_{1}}+\left(A y_{b}, y_{b}\right)^{x_{2}}<-\left(A y_{a}, y_{a}\right)^{a}+\left(A y_{b}, y_{b}\right)^{b} \leqq 0
$$

for each nonzero $\left[y_{a}, y_{b}\right] \in N_{a, b}$. Fixing $x_{1}, x_{2} \in(a, b)$ for the present, this in- 
equality shows that the $y^{1}$ components $\left[y_{a}^{1}\left(x_{1}\right), y_{b}^{1}\left(x_{2}\right)\right]$ of the solution pairs $\left[y_{a}, y_{b}\right]$ lying in $N_{a, b}$ span an $r$-dimensional linear subspace of the $2 r$-dimensional euclidean space; we denote this subspace by $N^{1}\left(x_{1}, x_{2}\right)$. Obviously

$$
-\left(A_{11}\left(x_{1}\right) y_{a}^{1}, y_{a}^{1}\right)+\left(A_{11}\left(x_{2}\right) y_{b}^{1}, y_{b}^{1}\right)<0
$$

for each nonzero vector pair $\left[y_{a}^{1}, y_{b}^{1}\right] \in N^{1}\left(x_{1}, x_{2}\right)$. We now apply Lemma 3.2 to the $2 r \times 2 r$ hermitian nonsingular matrix

$$
A_{11}\left(x_{1}, x_{2}\right)=\left(\begin{array}{cc}
-A_{11}\left(x_{1}\right) & 0 \\
0 & A_{11}\left(x_{2}\right)
\end{array}\right),
$$

having $r$ positive and $r$ negative eigenvalues. Let $P^{1}\left(x_{1}, x_{2}\right)$ be the $A_{11}\left(x_{1}, x_{2}\right)$ orthogonal complement to $N^{1}\left(x_{1}, x_{2}\right)$. Then, according to the lemma,

$$
-\left(A_{11}\left(x_{1}\right) z_{a}^{1}, z_{a}^{1}\right)+\left(A_{11}\left(x_{2}\right) z_{b}^{1}, z_{b}^{1}\right)>0
$$

for each nonzero vector pair $\left[z_{a}^{1}, z_{b}^{1}\right] \in P^{1}\left(x_{1}, x_{2}\right)$. Now Theorem 3.1 applied to the adjoint homogeneous system (3.2) asserts that the components $\left[z_{a}^{1}\left(x_{1}\right), z_{b}^{1}\left(x_{2}\right)\right]$, where $z_{a}$ and $z_{b}$ range over the solution pairs of (3.2), span the $2 r$-dimensional euclidean space. Hence if $P\left(x_{1}, x_{2}\right)$ denotes the set of solution pairs $\left[z_{a}, z_{b}\right]$ whose $z^{1}$ components $\left[z_{a}^{1}\left(x_{1}\right), z_{b}^{1}\left(x_{2}\right)\right]$ lie in $P^{1}\left(x_{1}, x_{2}\right)$, then $P\left(x_{1}, x_{2}\right)$ is an $r$-dimensional linear subspace whose nonzero elements satisfy

$$
-\left(A z_{a}, z_{a}\right)^{x_{1}}+\left(A z_{b}, z_{b}\right)^{x_{2}}<0 .
$$

It is clear from our earlier remarks that $P\left(x_{1}, x_{2}\right)$ is actually the set $P_{a, b}$ and that (3.16) is valid for all $x_{1}, x_{2} \in(a, b)$. Passing to the limit as $x_{1} \rightarrow a$ and $x_{2} \rightarrow b$, we obtain (3.14). Moreover, it is readily seen that if we had started with $P_{a, b}$ and proceeded as above, then we would have obtained $N^{1}\left(x_{1}, x_{2}\right)$ as the $A_{11}\left(x_{1}, x_{2}\right)$-orthogonal complement to $P^{1}\left(x_{1}, x_{2}\right)$ by Lemma 3.2 , and hence $N_{a, b}$ as the $A_{a, b}$-orthogonal complement to $P_{a, b}$. Finally the argument based on the relation (3.12) applied now to (3.14) shows that $P_{a, b} \subset G_{a, b}$.

If $\left[y_{a}, y_{b}\right]$ is a nonzero element of $N_{a, b}$, then as in (3.15)

$$
-\left(A y_{a}, y_{a}\right)^{x}+\left(A y_{b}, y_{b}\right)^{x}<0
$$

for each $x \in(a, b)$. As a consequence the $r$-dimensional subspace

$$
N^{\prime}(x) \equiv\left[\left[y_{b}(x), y_{a}(x)\right] ;\left[y_{a}, y_{b}\right] \in N_{a, b}\right]
$$

has only the zero element in common with

$$
N(x) \equiv\left[\left[y_{a}(x), y_{b}(x)\right] ;\left[y_{a}, y_{b}\right] \in N_{a, b}\right],
$$

and hence $N(x)$ and $N^{\prime}(x)$ together span the $2 r$-dimensional euclidean space for each $x \in(a, b)$. The same statement holds for

and

$$
P(x) \equiv\left[\left[z_{a}(x), z_{b}(x)\right] ;\left[z_{a}, z_{b}\right] \in P_{a, b}\right]
$$




$$
P^{\prime}(x) \equiv\left[\left[z_{b}(x), z_{a}(x)\right] ;\left[z_{a}, z_{b}\right] \in P_{a, b}\right] .
$$

Choosing $r$ linearly independent solutions $\left[y_{a, i}, y_{b, i}\right]$ in $N_{a, b}$ and $r$ linearly independent solutions $\left[z_{a, i}, z_{b, i}\right]$ in $P_{a, b}$, it follows that the $2 r \times 2 r$ matrices with column vectors

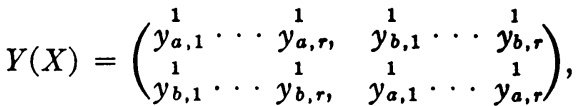

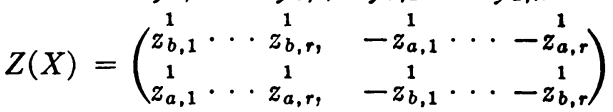

are nonsingular for each $x \in(a, b)$. Furthermore

$$
Q \equiv Z(x)^{*} A_{11}(x, x) Y(x)
$$

is nonsingular and by Theorem 3.2 independent of $x$. Since $N(x)$ and $N^{\prime}(x)$ are $A_{11}(x, x)$-orthogonal to $P(x)$ and $P^{\prime}(x)$ respectively, we see that $Q$ is of the form

$$
Q=\left(\begin{array}{cc}
Q_{11} & 0 \\
0 & Q_{11}
\end{array}\right)
$$

where each block is $r \times r$. We now set

$$
V(x)=Z(x)\left[Q^{*}\right]^{-1}
$$

from which we see that the first $r$ column vectors in $V(x)$ come from $P^{\prime}(x)$ and the last $r$ come from $P(x)$. In fact, $V(x)$ is composed precisely like $Z(x)$ from a single set of $r$ linearly independent solution pairs spanning $P_{a, b}$. To conserve on notation, we shall simply suppose that our original choice of solution pairs $\left[z_{a, i}, z_{b, i}\right]$ are those to be found in $V(x)$. In this case we have

$$
Z(x)^{*} A_{11}(x, x) Y(x)=I=A_{11}(x, x) Y(x) Z(x)^{*} \text {. }
$$

By employing the above choice of solution pairs, we now write down two forms of the solution to the equation

$$
\lambda y-(A y)_{x}-B y=f
$$

for functions $f \in L_{2}(a, b ; I)$ which vanish outside of $\left(a^{\prime}, b^{\prime}\right), a<a^{\prime}<b^{\prime}<b$.

$$
\begin{aligned}
y= & R_{1}(\lambda) f=y_{1}+y_{2} \\
=\left(\begin{array}{c}
R_{00}(\lambda) f_{0} \\
\theta
\end{array}\right) & -\sum_{i=1}^{r} y_{a, i} \int_{x}^{b}\left(f, z_{a, i}+z_{b, i}\right) d \xi \\
& -\sum_{i=1}^{r} y_{b, i} \int_{a}^{x}\left(f, z_{a, i}+z_{b, i}\right) d \xi
\end{aligned}
$$

and 


$$
\begin{aligned}
y=R_{2}(\lambda) f=y_{1}+y_{2} \\
=\left(\begin{array}{c}
R_{00}(\lambda) f_{0} \\
\theta
\end{array}\right)-\sum_{i=1}^{r}\left(y_{a, i}+y_{b, i}\right) \int_{x}^{b}\left(f, z_{b, i}\right) d \xi \\
\quad-\sum_{i=1}^{r}\left(y_{a, i}+y_{b, i}\right) \int_{a}^{x}\left(f, z_{a, i}\right) d \xi .
\end{aligned}
$$

In both of these expressions $R_{00}(\lambda)=\left(\lambda I-B_{00}\right)^{-1}$. Since $f(x)$ vanishes outside of $\left(a^{\prime}, b^{\prime}\right)$ it is clear in each case that all of the terms are well defined and that $A y=A y_{2}$ is absolutely continuous.

We proceed to verify that (3.20) solves (3.19). Making use of the fact that the $y_{a}^{\prime}$ 's and $y_{b}$ 's are solutions of (3.1), we have

$$
\begin{aligned}
\lambda y^{0}=B_{00} y^{0}-B_{01} y^{1} & =\left(\lambda I-B_{00}\right) R_{00}(\lambda) f^{0}+\left(\lambda y_{2}^{0}-B_{00} y_{2}^{0}-B_{01} y_{2}^{1}\right) \\
& =f^{0}+\theta^{0}=f^{0}
\end{aligned}
$$

and

$$
\begin{aligned}
\lambda y^{1}-\left(A_{11} y^{1}\right)_{x} & -B_{11} y^{1}-B_{10} y^{0} \\
= & -B_{10} R_{00}(\lambda) f^{0}+\left\{\lambda y_{2}^{1}-\left(A_{11} y_{2}^{1}\right)_{x}-B_{11} y_{2}^{1}-B_{10} y_{2}^{0}\right\} \\
= & -B_{10} R_{00}(\lambda) f^{0}-\sum_{i=1}^{r} A_{11} y_{a, i}^{1}\left(f, z_{a, i}+z_{b, i}\right) \\
& +\sum_{i=1}^{r} A_{11} y_{b, i}^{1}\left(f, z_{a, i}+z_{b, i}\right)
\end{aligned}
$$

for almost all $x$. If $z$ is a solution to the adjoint homogeneous system (3.2). the $z$-relation corresponding to (3.5) gives

$$
\begin{aligned}
(f, z) & =\left(f^{0}, z^{0}\right)+\left(f^{1}, z^{1}\right)=\left(f^{0}, R_{00}(\lambda)^{*} B_{10}^{*} z^{1}\right)+\left(f^{1}, z^{1}\right) \\
& =\left(B_{10} R_{00}(\lambda) f^{0}+f^{1}, z^{1}\right) .
\end{aligned}
$$

Further, one obtains the following identities directly from (3.18):

$$
\begin{aligned}
& -\sum_{i=1}^{r} A_{11} y_{a, i}^{1}\left(f^{1}, z_{b, i}^{1}\right)+\sum_{i=1}^{r} A_{11} y_{b, i}^{1}\left(f^{1}, z_{a, i}^{1}\right)=f^{1}, \\
& -\sum_{i=1}^{r} A_{11} y_{a, i}^{1}\left(f^{1}, z_{a, i}^{1}\right)+\sum_{i=1}^{r} A_{11} y_{b, i}^{1}\left(f^{1}, z_{b, i}^{1}\right)=\theta^{1} .
\end{aligned}
$$

Making use of (3.22) and (3.23) it is readily seen that

$$
\lambda y^{1}-\left(A_{11} y^{1}\right)_{x}-B_{11} y^{1}-B_{10} y^{0}=-B_{10} R_{00}(\lambda) f^{0}+\left[B_{10} R_{00}(\lambda) f^{0}+f^{1}\right]=f^{1}
$$

for almost all $x \in(a, b)$. The verification for (3.21) is essentially the same and is omitted. 
Before closing, a few remarks are in order on our choice of solution pairs $\left[y_{a, i}, y_{b, i}\right] \in N_{a, b}$ and $\left[z_{a, i}, z_{b, i}\right] \in P_{a, b}$ employed in (3.20) and (3.21). As described above, we kept our original set of $\left[y_{a, i}, y_{b, i}\right]$ 's and made use of $Q$ to obtain a suitable linear combination of the $\left[z_{a, i}, z_{b, i}\right]$ 's. It is clear that we could just as well have fixed the $\left[z_{a, i}, z_{b, i}\right]$ 's and used $Q$ to select a linear combination of the $\left[y_{a, i}, y_{l, i}\right]$ 's so as to satisfy (3.18), namely $U(x)=Y(x) Q^{-1}$.

To pursue this matter a bit further, suppose $N_{a, b}=N_{a} \times N_{b}$, where $N_{a}$ is a $p$-dimensional subspace of $C_{a}$ and $N_{b}$ an $n$-dimensional subspace of $C_{b}$. For a fixed $x \in(a, b)$ set

$$
N_{a}^{1}(x) \equiv\left[y^{1}(x) ; y \in N_{a}\right] \text { and } N_{b}^{1}(x) \equiv\left[y^{1}(x) ; y \in N_{b}\right] .
$$

We then obtain $P_{a}^{1}(x)$ and $P_{b}^{1}(x)$ as the $A_{11}(x)$-orthogonal complements to $N_{a}^{1}(x)$ and $N_{b}^{1}(x)$, respectively. According to Lemma 3.2, $P_{a}^{1}(x)$ will be $n$ dimensional, $P_{b}^{1}(x)$ will be $p$-dimensional, and

$$
\left(A_{11}(x) z_{a}, z_{a}\right)<0 \text { and }\left(A_{11}(x) z_{b}, z_{b}\right)>0
$$

for all nonzero vectors $z_{a} \in P_{a}^{1}(x)$ and $z_{b} \in P_{b}^{1}(x)$. As in the proof of Theorem 3.4 , the $n$-dimensional set of solutions of (3.2) whose $z^{1}(x)$ components span $P_{a}^{1}(x)$ is independent of $x$; we denote this set by $P_{a}$. The corresponding set of solutions whose $z^{1}(x)$ components span $P_{b}^{1}(x)$ will be called $P_{b}$. We then have

$$
\begin{aligned}
\left(A y_{a}, y_{a}\right)^{a} & \geqq 0, \quad\left(A y_{a} z_{a}\right)^{a}=0, \quad\left(A z_{a} z_{a}\right)^{a} & \leqq 0, \\
\left(A y_{b}, y_{b}\right)^{b} & \leqq 0, \quad\left(A y_{b}, z_{b}\right)^{b}=0, \quad\left(A z_{b}, z_{b}\right)^{b} & \geqq 0,
\end{aligned}
$$

for all $y_{a} \in N_{a}, z_{a} \in P_{a}, y_{b} \in N_{b}$, and $z_{b} \in P_{b}$. Moreover, since $P_{a} \times P_{b}$ is $r$-dimensional, it follows that this set is the $A_{a, b}$-orthogonal complement of $N_{a, b}$, that is, $P_{a, b}=P_{a} \times P_{b}$.

We now choose bases for these various sets of solutions:

$$
\begin{array}{ll}
N_{a}:\left[y_{a, i} ; i=1, \cdots, p\right], & N_{b}:\left[y_{b, i} ; i=p+1, \cdots, r\right], \\
P_{a}:\left[z_{a, i} ; i=p+1, \cdots, r\right], & P_{b}:\left[z_{b, i} ; i=1, \cdots, p\right],
\end{array}
$$

where the numeration is chosen so as to conform with the notation of (3.17) if we simply set all of the unlisted functions equal to the identically zero vector function. Defining the $2 r \times 2 r$ matrix $Q$ as before, we find that

$$
Q_{11}=\left(\begin{array}{cc}
Q_{11}^{1} & 0 \\
0 & Q_{11}^{2}
\end{array}\right)
$$

where $Q_{11}^{1}$ is $p \times p$ and $Q_{11}^{2}$ is $n \times n$. As a consequence $V(x)=Z(x)\left[Q^{*}\right]^{-1}$ is composed like $Z(x)$ with its first $p$ column vectors in $P_{b} \times \theta$ and the next $n$ column vectors in $\theta \times P_{a}$. On the other hand if we prefer to keep the original $P_{a}$ and $P_{b}$ basis vectors we may replace $Y(x)$ by $U(x)=Y(x) Q^{-1}$ which is again composed like $Y(x)$ in the sense that the first $p$ column vectors lie in $N_{a} \times \theta$ and 
the next $n$ lie in $\theta \times N_{b}$. Finally, if we factor $Q$ as

$$
Q=Q_{1} Q_{2}, \quad Q_{1}=\left(\begin{array}{llll}
Q_{11}^{1} & 0 & 0 & 0 \\
0 & I & 0 & 0 \\
0 & 0 & Q_{11}^{1} & 0 \\
0 & 0 & 0 & I
\end{array}\right), \quad Q_{2}=\left(\begin{array}{llll}
I & 0 & 0 & 0 \\
0 & Q_{11}^{2} & 0 & 0 \\
0 & 0 & I & 0 \\
0 & 0 & 0 & Q_{11}^{2}
\end{array}\right)
$$

and set $U(x)=Y(x) Q_{2}^{-1}$ and $V(x)=Z(x)\left[Q_{1}^{*}\right]^{-1}$, then (3.18) will be satisfied with $Y(x)$ replaced by $U(x)$ and $Z(x)$ replaced by $V(x)$. In this case we keep to our original choice of bases for $N_{a}$ and $P_{a}$ but modify the bases for $N_{b}$ and $P_{b}$. In a similar fashion we can keep our original choice of bases for $N_{b}$ and $P_{b}$ and modify only the bases for $N_{a}$ and $P_{a}$. In all of these cases of separated end conditions (3.20) and (3.21) coincide and are of the form

$$
\begin{aligned}
y=R(\lambda) f=y_{1}+y_{2} \\
=\left(\begin{array}{c}
R_{00}(\lambda) f^{0} \\
\theta
\end{array}\right)-\sum_{i=1}^{p} y_{a, i} \int_{x}^{b}\left(f, z_{b, i}\right) d \xi \\
-\sum_{i=p+1}^{r} y_{b, i} \int_{a}^{x}\left(f, z_{a, i}\right) d \xi .
\end{aligned}
$$

4. The operators $L_{1}$ and $M_{1}$. We now establish several basic properties of the operators $L_{1}$ and $M_{1}$, assuming, as before, that these operators are in normal form.

It is convenient at this point to introduce the extended operators $L_{\infty}$ and $M_{\infty}$ defined on the class of vector-valued functions

$$
\mathfrak{D}\left(L_{\infty}\right)=\mathfrak{D}\left(M_{\infty}\right)=[y ; A y \text { absolutely continuous }]
$$

as

$$
\begin{aligned}
L_{\infty} y & =(A y)_{x}+B y, \\
M_{\infty} z & =-(A z)_{x}+\left(B^{*}+A_{x}\right) z .
\end{aligned}
$$

The following relations, obtained directly from (2.12), hold amost everywhere in $(a, b)$ for $y, z \in \mathfrak{D}\left(L_{\infty}\right)=\mathfrak{D}\left(M_{\infty}\right)$ :

$$
\begin{aligned}
\left(L_{\infty} y_{1}, y_{2}\right)+\left(y_{1}, L_{\infty} y_{2}\right) & =\left(A y_{1}, y_{2}\right)_{x}+\left(\left(B+B^{*}+A_{x}\right) y_{1}, y_{2}\right), \\
\left(L_{\infty} y, z\right)-\left(y, M_{\infty} x\right) & =(A y, z)_{x}, \\
\left(M_{\infty} z_{1}, z_{2}\right)+\left(z_{1}, M_{\infty} z_{2}\right) & =-\left(A z_{1}, z_{2}\right)_{x}+\left(\left(B+B^{*}+A_{x}\right) z_{1}, z_{2}\right) .
\end{aligned}
$$

With the help of these relations we now establish certain limit theorems. It will be recalled that $H=L_{2}(a, b ; I)$ and we shall write $H^{2}=H \times H$. Also since $D \geqq I$ it is clear that $L_{2}(a, b ; D) \subset H \subset L_{2}\left(a, b ; D^{-1}\right)$, that $\|y\| \leqq\|y\|_{D}$ for $y \in H \cap L_{2}(a, b ; D)$, that $\|y\| \geqq\|y\|_{D^{-1}}$ for $y \in H \cap L_{2}\left(a, b ; D^{-1}\right)$, and that 
$|\langle y, z\rangle| \leqq\|y\|_{D}\|z\|_{D^{-1}}$ for $y \in L_{2}(a, b ; D)$ and $z \in L_{2}\left(a, b ; D^{-1}\right)$. These various spaces play an essential role in what follows and call attention to an important difference between the symmetric and the non-symmetric problem

Leмma 4.1. If $\left[y_{i}, L_{\infty} y_{i}\right]$ and $\left[z_{i}, M_{\infty} z_{i}\right] \in L_{2}(a, b ; D) \times L_{2}\left(a, b ; D^{-1}\right)$, $i=1,2$, then

$$
\left(A y_{1}, y_{2}\right)^{a}, \quad\left(A y_{1}, y_{2}\right)^{b}, \quad\left(A z_{1}, z_{2}\right)^{a}, \quad\left(A z_{1}, z_{2}\right)^{b}
$$

exist and are finite. Likewise if both $\left[y, L_{\infty} y\right]$ and $\left[z, M_{\infty} z\right] \in H^{2}$ or $L_{2}(a, b ; D)$ $\times L_{2}\left(a, b ; D^{-1}\right)$, then

$$
(A y, z)^{a} \text { and }(A y, z)^{b}
$$

exist and are finite.

Proof. Suppose first that $\left[y_{i}, L_{\infty} y_{i}\right] \in L_{2}(a, b ; D) \times L_{2}\left(a, b ; D^{-1}\right)$. Making use of the relation (4.4) we obtain

$$
\begin{aligned}
\left(A y_{1}, y_{2}\right)^{x}= & \left(A y_{1}, y_{2}\right)^{c}+\int_{c}^{x}\left[\left(L_{\infty} y_{1}, y_{2}\right)+\left(y_{1}, L_{\infty} y_{2}\right)\right] d \xi \\
& -\int_{c}^{x}\left(\left(B+B^{*}+A\right) y_{1}, y_{2}\right) d \xi, \quad a<c, x<b,
\end{aligned}
$$

and since both integrands belong to $L_{1}(a, b)$ the required limits evidently exist and are finite. The remaining assertions are proved in a similar fashion, the dual limits from (4.6) and the mixed limits from (4.5).

We note that if it is merely assumed that $\left[y, L_{\infty} y\right] \in H^{2}$, then the integrand in the first integral of (4.7) again belongs to $L_{1}(a, b)$. However the integrand in the second integral need not be summable, but since it is measurable and nonpositive we see that $y$ belongs to $L_{2}(c, b ; D)$ if and only if $(A y, y)^{b}$ exists and is finite whereas $y$ belongs to $L_{2}(a, c ; D)$ if and only if $(A y, y)^{a}$ exists and is finite. As was mentioned in the introduction, this is the reason for restricting $\mathfrak{D}\left(L_{1}\right)$ (and $\mathfrak{D}\left(M_{1}\right)$ ) to functions in $L_{2}(a, b ; D)$.

Lemma 4.2. Suppose $\beta(x)$ is a real-valued function of class $C^{(1)}$ on $(a, b)$ such that $0 \leqq \beta(x) \leqq 1, \beta(x)=0$ for all $x \leqq a^{\prime}$, and $\beta(x)=1$ for all $x \geqq b^{\prime}, a<a^{\prime}$ $<b^{\prime}<b$. If $\left\{y_{n} ; n=0,1,2, \cdots\right\} \subset \mathfrak{D}\left(L_{\infty}\right)$ with

$$
\left[y_{n}, L_{\infty} y_{n}\right] \rightarrow\left[y_{0}, L_{\infty} y_{0}\right] \text { in } H^{2}\left[\text { or in } L_{2}(a, b ; D) \times L_{2}\left(a, b ; D^{-1}\right)\right],
$$

then $\left\{u_{n}=\beta y_{n} ; n=0,1,2, \cdots\right\} \subset \mathfrak{D}\left(L_{\infty}\right)$ and

$$
\left[u_{n}, L_{\infty} u_{n}\right] \rightarrow\left[u_{0}, L_{\infty} u_{0}\right] \text { in } H^{2}\left[\text { or in } L_{2}(a, b ; D) \times L_{2}\left(a, b ; D^{-1}\right)\right]
$$

Similar results hold for $\alpha(x)=1-\beta(x)$ and for sequence $\left\{z_{n}\right\} \subset \mathfrak{D}\left(M_{\infty}\right)$.

Proof. Since $A u_{n}=\beta A y_{n}$ is absolutely continuous with $A y_{n}$, it is clear that $\left\{u_{n}\right\} \subset \mathcal{D}\left(L_{\infty}\right)$. One also sees immediately that 


$$
\int_{a}^{b}(E u, u) d x=\int_{a}^{b} \beta^{2}(E y, y) d x \leqq \int_{a}^{b}(E y, y) d x, \quad E=I[\text { or } D] .
$$

On the other hand

$$
L_{\infty} u=\beta L_{\infty} y+\beta_{x} A y
$$

so that

$$
\begin{aligned}
&\left\{\int_{a}^{b}\left(E^{-1} L_{\infty} u, L_{\infty} u\right) d x\right\}^{1 / 2} \leqq\left\{\int_{a}^{b}\left(E^{-1} L_{\infty} y, L_{\infty} y\right) d x\right\}^{1 / 2} \\
&+\left\{\int_{a}^{b}\left|\beta_{x}\right|^{2}\left|E^{-1}\right||A|^{2}(E y, y) d x\right\}^{1 / 2} \\
&\left.E=I \text { [or } D^{1}\right]
\end{aligned}
$$

Since $\beta_{x}$ vanishes outside of $\left[a^{\prime}, b^{\prime}\right]$ there exists a constant $M$ such that

$$
\int_{a}^{b}\left|\beta_{x}\right|^{2}\left|E^{-1}\right||A|^{2}(E y, y) d x \leqq M \int_{a}^{b}(E y, y) d x .
$$

The inequalities (4.8), (4.9), and (4.10) together show that $\left[u_{n}, L_{\infty} u_{n}\right]$ $\rightarrow\left[u_{0}, L_{\infty} u_{0}\right]$ in $H^{2}$ [or in $\left.L_{2}(a, b ; D) \times L_{2}\left(a, b ; D^{-1}\right)\right]$.

Lemma 4.3. Suppose $\left\{y_{n} ; n=0,1,2, \cdots\right\} \subset \mathfrak{D}\left(L_{\infty}\right),\left\{z_{n} ; n=0,1,2, \cdots\right\}$ $\mathcal{C D}\left(M_{\infty}\right)$, and that

$$
\left[y_{n}, L_{\infty} y_{n}\right] \rightarrow\left[y_{0}, L_{\infty} y_{0}\right] \text { and }\left[z_{n}, M_{\infty} z_{n}\right] \rightarrow\left[z_{0}, M_{\infty} z_{0}\right]
$$

in $H^{2}$ or in $L_{2}(a, b ; D) \times L_{2}\left(a, b ; D^{-1}\right)$. Then

$$
\lim _{n \rightarrow \infty}\left(A y_{n} z_{n}\right)^{x}=\left(A y_{0}, z_{0}\right)^{x} \text { for } x=a, b .
$$

Proof. We make use of the previous lemma, setting

$$
u_{n}=\beta y_{n} \quad \text { and } \quad v_{n}=\beta z_{n} .
$$

Since the new sequences have the same convergence properties as the original sequences, we have by $(4.5)$

$$
\begin{aligned}
\left(A y_{n}, z_{n}\right)^{b} & =\left(A u_{n}, v_{n}\right)^{b}=\int_{a}^{b}\left[\left(L_{\infty} u_{n}, v_{n}\right)-\left(u_{n}, M_{\infty} v_{n}\right)\right] d x \\
& \rightarrow \int_{a}^{b}\left[\left(L_{\infty} u_{0}, v_{0}\right)-\left(u_{0}, M_{\infty} v_{0}\right)\right] d x=\left(A u_{0}, v_{0}\right)^{b}=\left(A y_{0}, z_{0}\right)^{b} .
\end{aligned}
$$

A similar proof applies at $x=a$ if we replace $\beta(x)$ by $\alpha(x)=1-\beta(x)$.

Lemma 4.4. Suppose $\left\{y_{n} ; n=0,1,2, \cdots\right\} \subset \mathcal{D}\left(L_{1}\right)$ and 


$$
\left[y_{n}, L_{1} y_{n}\right] \rightarrow\left[y_{0}, L_{1} y_{0}\right] \text { in } H^{2} \text {. }
$$

Then

$$
\begin{aligned}
& \liminf _{n \rightarrow \infty}\left(A y_{n}, y_{n}\right)^{b} \geqq\left(A y_{0}, y_{0}\right)^{b}, \\
& \limsup _{n \rightarrow \infty}\left(A y_{n}, y_{n}\right)^{a} \leqq\left(A y_{0}, y_{0}\right)^{a} .
\end{aligned}
$$

The corresponding results hold for $M_{1}$.

Remark. As we shall see in Lemma 5.3, the left members in (4.11) actually converge to the right members.

Proof. Again making use of Lemma 4.2 and setting $u_{n}=\beta y_{n}$, we have by

$$
\begin{aligned}
\left(A y_{n}, y_{n}\right)^{b}=\left(A u_{n}, u_{n}\right)^{b}=\int_{a}^{b}\left[\left(L_{1} u_{n}, u_{n}\right)+\left(u_{n}, L_{1} u_{n}\right)\right] d x & \\
& -\int_{a}^{b}\left(\left(B+B^{*}+A_{x}\right) u_{n}, u_{n}\right) d x .
\end{aligned}
$$

Since $\left[u_{n}, L_{1} u_{n}\right] \rightarrow\left[u_{0}, L_{1} u_{0}\right]$ in $H^{2}$, we see that

$$
\int_{a}^{b}\left[\left(L_{1} u_{n}, u_{n}\right)+\left(u_{n}, L_{1} u_{n}\right)\right] d x \rightarrow \int_{a}^{b}\left[\left(L_{1} u_{0}, u_{0}\right)+\left(u_{0}, L_{1} u_{0}\right)\right] d x .
$$

Further it follows from Fatou's lemma that

$$
-\int_{a}^{b}\left(\left(B+B^{*}+A_{x}\right) u_{0}, u_{0}\right) d x \leqq \liminf _{n \rightarrow \infty}-\int_{a}^{b}\left(\left(B+B^{*}+A_{x}\right) u_{n}, u_{n}\right) d x .
$$

Combining these two observations with the expression (4.12), we obtain the first inequality in (4.11) and the second is proved in a similar fashion

We note that Lemmas 4.3 and 4.4 also apply to functions which behave in the prescribed manner in some neighborhood of the point $x=b$ [or $a]$ at which the limit is taken. It suffices in the proof to choose $\beta(x)$ [or $\alpha(x)$ ] so that it vanishes outside of this neighborhood.

The estimates established in the next lemma are basic.

Lemma 4.5. Suppose $y \in \mathfrak{D}\left(L_{\infty}\right)$ is square integrable on each compact subinterval of $(a, b)$, that $-(A y, y)^{a}+(A y, y)^{b} \leqq 0$, and for some $\lambda>0$ that the function $f \equiv \lambda y-L_{\infty} y \in H$. Then $y \in \mathfrak{D}\left(L_{1}\right)$,

$$
\lambda\|y\| \leqq\|f\|,
$$

and

$$
c(\lambda)\|y\| \leqq c(\lambda)\left\{\int_{a}^{b}(D y, y) d x\right\}^{1 / 2} \leqq\left\{\int_{a}^{b}\left(D^{-1} f, f\right) d x\right\}^{1 / 2} \leqq\|f\|
$$


where $c(\lambda)=\min (\lambda, 1 / 2)$. On the other hand, if $y \in \mathcal{D}\left(L_{\infty}\right)$ belongs to $L_{2}\left(a^{\prime}, b^{\prime} ; D\right)$ for all $a<a^{\prime}<b^{\prime}<b$, if $-(A y, y)^{a}+(A y, y)^{b} \leqq 0$, and if $f \in L_{2}\left(a, b ; D^{-1}\right)$, then $y \in L_{2}(a, b ; D)$ and the first two inequalities in (4.14) remain valid.

Proof. It follows directly from (4.4) that

$$
2 \lambda(y, y)-(f, y)-(y, f)=(A y, y)_{x}+\left(\left(B+B^{*}+A_{x}\right) y, y\right)
$$

for almost all $x$. This relation represents $\left(\left(B+B^{*}+A_{x}\right) y, y\right)$ as a sum of terms each summable over $\left(a^{\prime}, b^{\prime}\right)$ and therefore $\left(\left(B+B^{*}+A_{x}\right) y, y\right) \in L_{1}\left(a^{\prime}, b^{\prime}\right)$. Making use of condition (D) and the Schwarz inequality, we obtain

$$
\begin{aligned}
2 \lambda \int_{a^{\prime}}^{b^{\prime}}(y, y) d x \leqq & 2 \lambda \int_{a^{\prime}}^{b^{\prime}}(y, y) d x-\int_{a^{\prime}}^{b^{\prime}}\left(\left(B+B^{*}+A_{x}\right) y, y\right) d x \\
= & \int_{a^{\prime}}^{b^{\prime}}[(f, y)+(y, f)] d x+\left[(A y, y)^{b^{\prime}}-(A y, y)^{a^{\prime}}\right] \\
\leqq & 2\left\{\int_{a^{\prime}}^{b^{\prime}}(f, f) d x\right\}^{1 / 2}\left\{\int_{a^{\prime}}^{b^{\prime}}(y, y) d x\right\}^{1 / 2} \\
& +\left[(A y, y)^{b^{\prime}}-(A y, y)^{a^{\prime}}\right] .
\end{aligned}
$$

If we now divide the first and last members of this series of inequalities by $\left\{\int_{a^{\prime}}^{b^{\prime}}(y, y) d x\right\}^{1 / 2}$ and pass to the limit as $a^{\prime} \rightarrow a$ and $b^{\prime} \rightarrow b$, we get (4.13). This shows that $y \in H$ and, as a consequence, $L_{\infty} y=\lambda y-f \in H$. In addition, (4.15) now holds with $\left(a^{\prime}, b^{\prime}\right)$ replaced by $(a, b)$, and it follows from this that $y \in L_{2}(a, b ; D)$ so that $y \in \mathfrak{D}\left(L_{1}\right)$. Finally we note that the extreme inequalities in (4.14) are simple consequences of

$$
D=I-\left(B+B+A_{x}\right) \geqq I \text { and } D^{-1} \leqq I,
$$

whereas (4.15) implies

$$
\begin{aligned}
c(\lambda) \int_{a}^{b}(D y, y) d x & \leqq \int_{a}^{b}\left|\left(D^{-1 / 2} f, D^{1 / 2} y\right)\right| d x \\
& \leqq\left\{\int_{a}^{b}\left(D^{-1} f, f\right) d x\right\}^{1 / 2}\left\{\int_{a}^{b}(D y, y) d x\right\}^{1 / 2},
\end{aligned}
$$

from which the middle inequality in (4.14) follows.

To prove the second assertion of the lemma, we make use of (4.15) to obtain

$$
\begin{aligned}
c(\lambda) \int_{a^{\prime}}^{b^{\prime}}(D y, y) d x \leqq & \left\{\int_{a^{\prime}}^{b^{\prime}}\left(D^{-1} f, f\right) d x\right\}^{1 / 2}\left\{\int_{a^{\prime}}^{b}(D y, y) d x\right\}^{1 / 2} \\
& +\left[(A y, y)^{b^{\prime}}-(A y, y)^{a^{\prime}}\right] .
\end{aligned}
$$

Dividing now by $\left\{\int_{a^{\prime}}^{b^{\prime}}(D y, y) d x\right\}^{1 / 2}$ and passing to the limit, we obtain the 
middle inequality in (4.14) from which it follows that $y \in L_{2}(a, b ; D)$. The first inequality in (4.14) is obtained just as before.

COROLlary. There are no nontrivial dissipative solutions of $\lambda y-L_{1} y=\theta$ (in $\mathfrak{D}\left(L_{1}\right)$ ) for $\lambda>0$.

This is an immediate consequence of (4.13).

We are now in a position to show that (3.20) and (3.21) are both representations of the resolvent $R(\lambda ; L)$ of a suitably chosen restriction $L$ of $L_{1}$. We have

Theorem 4.1. For fixed $\lambda>0$, let $P_{a, b}$ be any $r$-dimensional subspace of solution pairs $\left[z_{a}, z_{t}\right]$ of (3.2) such that

$$
-\left(A z_{a}, z_{a}\right)+\left(A z_{b}, z_{b}\right)^{b} \geqq 0, \quad\left[z_{a}, z_{b}\right] \in P_{a, b} .
$$

Further define $L$ to be the restriction of $L_{1}$ with domain

(4.17) $\mathfrak{D}(L)=\left[y ; y \in \mathfrak{D}\left(L_{1}\right),-\left(A y, z_{a}\right)^{a}+\left(A y, z_{b}\right)^{b}=0\right.$ for all $\left.\left[z_{a}, z_{b}\right] \in P_{a, b}\right]$.

Then $L$ is a dissipative operator with dense domain, having a resolvent at $\lambda$ which can be represented by both (3.20) and (3.21); here the solution pairs $\left[z_{a, i}, z_{b, i}\right]$ span $P_{a, b}$ and the $\left[y_{a, i}, y_{l, i}\right]$ are solution pairs of (3.1) spanning the $A_{a, b}$-orthogonal complement to $P_{a, b}$, namely,

$$
\begin{aligned}
N_{a, b}=\left[\left[y_{a}, y_{b}\right]\right. & ; y_{a}, y_{b} \text { solutions of }(3.1) \text { such that } \\
& \left.-\left(A y_{a}, z_{a}\right)^{a}+\left(A y_{b}, z_{b}\right)^{b}=0 \text { for all }\left[z_{a}, z_{b}\right] \in P_{a, b}\right] ;
\end{aligned}
$$

these bases are chosen so as to satisfy the relation (3.18). In addition, $\lambda\|R(\lambda ; L)\|$ $\leqq 1$.

Proof. As we have seen in $\S 3$, for $f \in H$ and vanishing outside of $\left(a^{\prime}, b^{\prime}\right)$, $a<a^{\prime}<b^{\prime}<b$, the operators $R_{1}(\lambda)$ and $R_{2}(\lambda)$ are well defined and solve the equation (3.19). For $y=R_{1}(\lambda) f=y_{1}+y_{2}$,

$$
\begin{aligned}
& y_{1}=\left(\begin{array}{c}
R_{00}(\lambda) f^{0} \\
\theta
\end{array}\right), \\
& y_{2}=\left\{\begin{array}{lr}
\sum_{i=1}^{r} \gamma_{i} y_{a, i}, & a<x<b, a^{\prime}, \\
\sum_{i=1}^{r} \gamma_{i} y_{b, i}, & x>b^{\prime}
\end{array}\right.
\end{aligned}
$$

where $\gamma_{i}=-\int_{a}^{b}\left(f, z_{a, i}+z_{b, i}\right) d x$. Now $y_{1} \in H$ but does not enter in the computation of either $(A y, y)$ or $(A y, z)$ since its $y^{1}$ component vanishes. On the other hand it is evident that $y_{2}$ is square integrable on each compact subinterval of $(a, b)$ and it is seen from $(4.19)$ that $\left[y_{2}\left(x_{1}\right), y_{2}\left(x_{2}\right)\right]$ coincides with a solution pair in $N_{a, b}$ for $x_{1}<a^{\prime}$ and $x_{2}>b^{\prime}$. Consequently 


$$
-(A y, y)^{a}+(A y, y)^{b} \leqq 0
$$

and

$$
-\left(A y, z_{a}\right)^{a}+\left(A y, z_{b}\right)^{b}=0 \text { for all }\left[z_{a}, z_{b}\right] \in P_{a, b} .
$$

The hypothesis of Lemma 4.5 is therefore satisfied. Hence $y \in \mathfrak{D}\left(L_{1}\right)$ and this together with (4.21) shows that $y \in \mathfrak{D}(L)$, defined as in (4.17). The lemma also implies that $\lambda\left\|R_{1}(\lambda) f\right\| \leqq\|f\|$ for such $f$.

Similarly for $y=R_{2}(\lambda) f=y_{1}+y_{2}, f \in H$ and vanishing outside of $\left(a^{\prime}, b^{\prime}\right)$, we have

$$
\begin{aligned}
& y_{1}=\left(\begin{array}{cr}
R_{00}(\lambda) f^{0} \\
\theta
\end{array}\right), \\
& y_{2}=\left\{\begin{array}{lr}
-\sum_{i=1}^{r}\left(y_{a, i}+y_{b, i}\right) \int_{a}^{b}\left(f, z_{b, i}\right) d x, & a<x<b, a^{\prime}, \\
-\sum_{i=1}^{r}\left(y_{a, i}+y_{b, i}\right) \int_{a}^{b}\left(f, z_{a, i}\right) d x, & x>b^{\prime} .
\end{array}\right.
\end{aligned}
$$

Thus for $x_{1}<a^{\prime}$ and $x_{2}>b^{\prime}$

$$
\begin{aligned}
-\left(A y, z_{a, j}\right)^{x_{1}}+\left(A y, z_{b, j}\right)^{x_{2}} & =\sum_{i=1}^{r}\left[\left(A y_{a, i}, z_{a, j}\right)^{x_{1}}+\left(A y_{b, i}, z_{a, j}\right)^{x_{1}}\right] \int_{a}^{b}\left(f, z_{b, i}\right) d x \\
& -\sum_{i=1}^{r}\left[\left(A y_{a, i}, z_{b, j}\right)^{x_{2}}+\left(A y_{b, i}, z_{b, j}\right)^{x_{2}}\right] \int_{a}^{b}\left(f, z_{a, i}\right) d x .
\end{aligned}
$$

According to (3.22), $\left(f, z_{a}\right)=\left(h^{1}, z_{a}^{1}\right)$ and $\left(f, z_{b}\right)=\left(h^{1}, z_{b}^{1}\right)$, where we have set $h^{1}=B_{10} R_{00}(\lambda) f^{0}+f^{1}$. On the other hand $(A y, z)^{x}$ is independent of $x$ if $y$ is a solution of (3.1) and $z$ a solution of (3.2). We can therefore bring the above expressions of this sort under the integral sign, replacing $x_{1}$ and $x_{2}$ by the variable of integration, $x$. Making use of these facts together with the second identity in (3.23) we get

$$
\begin{aligned}
\sum_{i=1}^{r}\left(A y_{b, i}, z_{a, j}\right)^{x_{1}} \int_{a}^{b}\left(f, z_{b, i}\right) d x & =\int_{a}^{b}\left(\sum_{i=1}^{r} A_{11} y_{b, i}^{1}\left(h^{1}, z_{b, i}^{1}\right), z_{a, j}^{1}\right) d x \\
& =\int_{a}^{b}\left(\sum_{i=1}^{r} A_{11} y_{a, i}^{1}\left(h^{1}, z_{a, i}^{1}\right), z_{a, i}^{1}\right) d x \\
& =\sum_{i=1}^{r}\left(A y_{a, i}, z_{a, j}\right)^{x_{1}} \int_{a}^{b}\left(f, z_{a, i}\right) d x,
\end{aligned}
$$

and likewise

$$
\sum_{i=1}^{r}\left(A y_{a, i}, z_{b, j}\right)^{x_{2}} \int_{a}^{b}\left(f, z_{a, i}\right) d x=\sum_{i=1}^{r}\left(A y_{b, i}, z_{b, j}\right)^{x_{2}} \int_{a}^{b}\left(f, z_{b, i}\right) d x .
$$


Consequently

$$
\begin{aligned}
-\left(A y, z_{a, j}\right)^{x_{1}}+\left(A y, z_{b, j}\right)^{x_{2}} & \\
& =\sum_{i=1}^{r}\left[\left(A y_{a, i}, z_{a, j}\right)^{x_{1}}-\left(A y_{b, i}, z_{b, j}\right)^{x_{2}}\right] \int_{a}^{b}\left(f, z_{a, i}+z_{b, i}\right) d x
\end{aligned}
$$

which vanishes since $\left[y_{a, i}, y_{b, i}\right]$ is $A_{a, b}$-orthogonal to $\left[z_{a, j}, z_{b, j}\right]$. It follows from this that $\left[y_{2}\left(x_{1}\right), y_{2}\left(x_{2}\right)\right]$ coincides with a solution pair in $N_{a, b}$ for $x_{1}<a^{\prime}$ and $x_{2}>b^{\prime}$. Arguing as above we see that $y \in \mathfrak{D}(L)$ and that $\lambda\left\|R_{2}(\lambda) f\right\| \leqq\|f\|$. We note that $u=R_{1}(\lambda) f-R_{2}(\lambda) f$ satisfies the homogeneous Equation (3.1) and that $\left[u\left(x_{1}\right), u\left(x_{2}\right)\right]$ coincides with a solution pair in $N_{a, b}$ for $x_{1}<a^{\prime}$ and $x_{2}>b^{\prime}$. As a consequence $u$ is a dissipative solution of $\lambda u-L_{1} u=\theta$ and by the corollary to Lemma $4.5, u \equiv \theta$; in other words $R_{1}(\lambda) f=R_{2}(\lambda) f$ if $f$ vanishes near $a$ and $b$.

From this point on the proof is the same for both operators. Let $f_{0}$ be an arbitrary element of $H$ and choose the sequence $\left\{f_{n}\right\} \subset H$ to consist of vector-valued functions vanishing near $a$ and $b$ and such that $f_{n} \rightarrow f_{0}$ in $H$. It is clear from the boundedness of $R_{i}(\lambda)$ that the sequence $\left\{y_{n} \equiv R_{i}(\lambda) f_{n}\right\}$ converges in $H$ to a function, say $y_{0}$, which is given explicitly by (3.21) as $y_{0}$ $=R_{2}(\lambda) f_{0}$. (We note that the expression (3.20) corresponding to $R_{1}(\lambda) f_{0}$ need not be well defined.) Thus $A y_{0}$ is absolutely continuous and $L_{\infty} y_{0}=\lambda y_{0}-f_{0} \in H$. It is clear that the extended operators, $R_{i}(\lambda)$, have the same bound as before. Moreover the inequality (4.14) shows that $y_{n} \rightarrow y_{0}$ even in $L_{2}(a, b ; D)$ so that $y_{0} \in L_{2}(a, b ; D)$. It follows that $y_{0} \in \mathfrak{D}\left(L_{1}\right)$. Finally $L_{1} y_{n}=\lambda y_{n}-f_{n} \rightarrow \lambda y_{0}-f_{0}$ $=L_{1} y_{0}$ in $H$ and applying Lemmas 4.3 and 4.4 we see that (4.20) and (4.21) continue to hold for $y_{0}$. This proves that $y_{0} \in \mathfrak{D}(L)$ and hence that $R_{i}(\lambda)$ is a right inverse for $\lambda I-L$. It remains to show that the range of $R_{i}(\lambda)$ actually fills out $\mathfrak{D}(L)$. To this end let $y$ be an arbitrary element of $\mathfrak{D}(L)$ and set $f=\lambda y-L y$ and $y_{0}=R_{i}(\lambda) f$. Then $u \equiv y-y_{0} \in \mathcal{D}(L)$ is a solution of the homogeneous Equation (3.1) and by (4.17) $[u, u]$ is $A_{a, b}$-orthogonal to $P_{a, b}$. It follows from Theorem 3.4 that $[u, u] \in N_{a, b}$ and hence that $u$ itself is a dissipative solution. The corollary to Lemma 4.5 now asserts that $u \equiv \theta$ and this shows that $y=R_{i}(\lambda) f$ lies in the range of $R_{i}(\lambda)$. Thus $R_{i}(\lambda)$ is also a left inverse for $\lambda I-L$ and is therefore the resolvent of $L$ at $\lambda$. As we have seen, (4.20) holds for each element in the range of $R_{i}(\lambda)$ and hence for each element in $\mathfrak{D}(L)$ so that $L$ is a dissipative operator. Finally it is clear that $\mathfrak{D}(L)$ contains the class of continuously differentiable functions vanishing near $a$ and $b$, and since these functions are dense in $H$ the same is true of $\mathfrak{D}(L)$. This concludes the proof of Theorem 4.1.

It is convenient to bring the operators $R_{1}(\lambda)$ and $R_{2}(\lambda)$ into a somewhat more tractable form. This can be accomplished by a suitable choice of bases for $N_{a, b}$ and $P_{a, b}$; as we have already remarked at the close of $\S 3$, there is considerable latitude in the choice of these bases. 
In the case of $R_{2}(\lambda)$ we proceed as follows: Let $N_{1}$ be the subspace of $N_{a, b}$ for which both of the solutions in $\left[y_{a}, y_{b}\right]$ lie in $L_{2}(a, b ; D)$, let $N_{2}$ be the subspace of $N_{a, b}$ for which the $y_{b}$ solution lies in $L_{2}(a, b ; D)$, and let $N_{3}$ be the subspace of $N_{a, b}$ for which the $y_{a}$ solution lies in $L_{2}(a, b ; D)$. It is clear that $N_{1}=N_{2} \cap N_{3}$. We now choose a first set of $s_{1}$ linearly independent solution pairs $\left[y_{a, i}, y_{b, i}\right]$ to span $N_{1}$, a second set of $s_{2}$ solution pairs which together with the first set form a basis for $N_{2}$, a third set of $s_{3}$ solution pairs which together with the first set form a basis for $N_{3}$, and a last set of $s_{4}$, solution pairs which complete the basis for $N_{a, b}$. We then choose a basis of solution pairs $\left[z_{a, i}, z_{b, i}\right]$ for $P_{a, b}$ as before so as to satisfy (3.18). Suppose next that $f \in H$ vanishes outside of $\left[a^{\prime}, b^{\prime}\right] \subset(a, b)$. It was shown in Theorem 4.1 that $y=R_{2}(\lambda) f \in \mathfrak{D}(L)$ and $a$ fortiori that $y \in L_{2}(a, b ; D)$. In particular

$$
y(x)=-\sum_{i=1}^{r}\left[y_{a, i}(x)+y_{b, i}(x)\right] \int_{a}^{b}\left(f, z_{b, i}\right) d x, \quad x<a^{\prime},
$$

lies in $L_{2}\left(a, a^{\prime} ; D\right)$. Now the solutions $\left[y_{a, i}+y_{b, i} ; i=1, \cdots, r\right]$ are linearly independent. Otherwise there would be constants $\left\{\gamma_{i}\right\}$ not all zero such that $\sum_{i=1}^{r} \gamma_{i}\left[y_{a, i}+y_{b, i}\right]=\theta$ almost everywhere, and setting $y_{0}=\sum \gamma_{i} y_{a, i}$ $=-\sum \gamma_{i} y_{b, i}$ we see that $\left[y_{0}, y_{0}\right] \in N_{a, b}$. Consequently $-\left(A y_{0}, y_{0}\right)^{a}+\left(A y_{0}, y_{0}\right)^{b}$ $\leqq 0$ which can happen, according to Theorem 3.2, only if $y_{0}$ is the trivial solution of (3.1). Thus $\sum \gamma_{i}\left[y_{a, i}, y_{b, i}\right]=[\theta, \theta]$ almost everywhere, contrary to the $\left[y_{a, i}, y_{b, i}\right]$ forming a basis. Thus the terms in the above expression for $y(x)$ are linearly independent and, since $y \in L_{2}\left(a, a^{\prime} ; D\right)$, the sum can extend only over $s_{1} \cup s_{2}$. In view of the fact that our choice of $f^{\prime} \mathrm{s}$ is dense in $L_{2}\left(a^{\prime}, b^{\prime} ; I\right)$, this implies that $z_{b, i} \equiv \theta$ for $i \notin s_{1} \cup s_{2}$. A similar argument for the $b$-end shows that $z_{a, i} \equiv \theta$ for $i \notin s_{1} \cup s_{3}$. Since the $\left[z_{a, i}, z_{b, i}\right]$ are linearly independent, both $z_{a, i}$ and $z_{b, i}$ can not be zero solutions of (3.2) and hence the set $s_{4}$ must be empty. Thus $R_{2}(\lambda)$ takes the form

$$
\begin{aligned}
R_{2}(\lambda) f=\left(\begin{array}{c}
R_{00}(\lambda) f^{0} \\
\theta
\end{array}\right) & -\sum_{i \in s_{1} \cup}\left(y_{a, i}+y_{b, i}\right) \int_{x}^{b}\left(f, z_{b, i}\right) d \xi \\
& -\sum_{i \in s_{1} \cup s_{3}}\left(y_{a, i}+y_{b, i}\right) \int_{a}^{x}\left(f, z_{a, i}\right) d \xi .
\end{aligned}
$$

For the case of $R_{1}(\lambda)$ we consider the solution pairs $\left[z_{a}, z_{b}\right] \subset P_{a, b}$, splitting $P_{a, b}$ into parts $P_{1}, P_{2}, P_{3}$, and $P_{4}$ which are defined as the analogues of $N_{1}, N_{2}, N_{3}$ and $N_{4}$ respectively. We then choose a basis for $P_{a, b}$ so that the first set of $t_{1}$ forms a basis for $P_{1}$, etc.; after which a basis for $N_{a, b}$ is chosen so as to satisfy (3.18). For functions $f, g \in H$ which vanish near $a$ and $b$, we have

$$
\left(g, R_{1}(\lambda) f\right)=\left(R_{1}(\lambda)^{*} g, f\right)
$$

from which we read off that 


$$
\begin{aligned}
R_{1}(\lambda)^{*} g=\left(\begin{array}{c}
R_{00}(\lambda)^{*} g^{0} \\
\theta
\end{array}\right) & -\sum_{i=1}^{r}\left(z_{a, i}+z_{b, i}\right) \int_{x}^{b}\left(g, y_{b, i}\right) d \xi \\
& -\sum_{i=1}^{r}\left(z_{a, i}+z_{b, i}\right) \int_{a}^{x}\left(g, y_{a, i}\right) d \xi .
\end{aligned}
$$

According to Theorem 4.1, $R_{1}(\lambda)^{*}=R(\lambda ; M)$ where $M$ is a dissipative restriction of $M_{1}$ with

$$
\begin{aligned}
& \mathfrak{D}(M)=\left[z ; z \in \mathfrak{D}\left(M_{1}\right),-\left(A z, y_{a}\right)^{a}+\left(A z, y_{b}\right)^{b}=0\right. \\
& \text { for all } \left.\left[y_{a}, y_{b}\right] \in N_{a, b}\right] .
\end{aligned}
$$

Since the operator $R_{1}(\lambda)$ * is of the same form as $R_{2}(\lambda)$, the previous argument applies and we see that $y_{b, i} \equiv \theta$ for $i \notin t_{1} \cup t_{2}, y_{a, i} \equiv \theta$ for $i \notin t_{1} \cup t_{3}$, and that $t_{4}$ is empty. Consequently with this choice of bases for $P_{a, b}$, the expression for $R_{1}(\lambda)$ given in (3.20) is well defined for all $f \in H$.

We also have

THEOREM 4.2. Let $L \subset L_{1}$ and $M \subset M_{1}$ be defined by (4.17) and (4.24) respectively. Then $L^{*}=M$ and $M^{*}=L$.

Proof. Since $R_{1}(\lambda)$ is a representation for $R(\lambda ; L)$ by Theorem 4.1 , the above argument shows that $R(\lambda ; L)^{*}=R(\lambda ; M)$. On the other hand it is known (see R. S. Phillips [13]) that $R(\lambda ; L)^{*}=R\left(\lambda ; L^{*}\right.$ ) and from this it follows that $L^{*}=M$. A similar argument shows that $M^{*}=L$.

5. Boundary behavior. In the previous section we started with a fixed $\lambda>0$ and proceeded to define certain dissipative restrictions of $L_{1}$ (and $M_{1}$ ) whose resolvents exist and satisfy an inequality of the Hille-Yosida type for the given $\lambda$. It should be noted that the domains of these restrictions are defined in terms of solutions to the adjoint homogeneous equation (3.2) which again depends on the given $\lambda$. Now, in order to satisfy the Hille-Yosida condition it will be necessary for a given dissipative restriction to be defined in this way for all sufficiently large $\lambda\left({ }^{7}\right)$. This provides the motivation for the

$\left({ }^{7}\right)$ One can show that the Hille-Yosida condition is satisfied by a dissipative operator $L$ defined as in (4.17) by means of the following direct argument. We suppose, then, that $\mathfrak{D}(L)$ is defined as the $A_{a, b}$-orthogonal complement in $\mathfrak{D}\left(L_{1}\right)$ of the solution pairs $P_{a, b}$ to the homogeneous adjoint equation (3.2) for a given $\lambda=\lambda_{0}$ and to emphasize this dependence on $\lambda_{0}$ we shall denote this set by $P_{a, b}\left(\lambda_{0}\right)$. Let $\lambda>0$ be such that $\left|\lambda-\lambda_{0}\right|<\lambda_{0}$ and set $f_{i}(x)=y_{a, i}(x)$, $a<x<c, f_{i}(x)=y_{b, i}(x), c<x<b$, for $i=1,2, \cdots, r$. As in the proof of Theorem 5.1, the integral equation $u-\left(\lambda_{0}-\lambda\right) R\left(\lambda_{0} ; L\right) u=f_{i}$ will have a solution, say $u_{i}$, and the solutions $u_{1}, u_{2}, \cdots, u_{r}$ will be linearly independent. Moreover these functions $u_{i}$ satisfy (3.1) almost everywhere and $A u_{i}$ is absolutely continuous except perhaps at the point $x=c$. Consequently each of the functions $u_{a, i}(x)=u_{i}(x), a<x<c, u_{b, i}(x)=u_{i}(x), c<x<b$, can be extended to the entire interval $(a, b)$ so as to be a solution in the accepted sense of $(3.1)$ on $(a, b)$. Now the resulting solution pairs $\left[u_{a, i}, u_{b, i}\right]$ will be linearly independent; in fact, if $\sum \gamma_{i}\left[u_{a, i}, u_{b, i}\right]=[\theta, \theta]$ almost everywhere, then $\sum \gamma_{i} u_{i}(x)=\theta$ almost everywhere and this is impossible. We see that the integral equation expresses $u_{i}$ as the sum of a function in $\mathfrak{D}(L)$ plus $y_{a, i}$ for $a<x<c$ and plus $y_{b, i}$ for 
present section whose end result is a complete characterization of the pertinant boundary behavior of the functions in $\mathfrak{D}\left(L_{1}\right)$ (and $\mathfrak{D}\left(M_{1}\right)$ ).

It will be recalled that the boundary conditions on $\mathfrak{D}(L)$ are of the form

$$
-\left(A y, z_{a}\right)^{a}+\left(A y, z_{b}\right)^{b}=0
$$

for a set of solution pairs $\left[z_{a}, z_{b}\right] \subset G$. It is clear that only the behavior of $z_{a}$ near $a$ and of $z_{b}$ near $b$ effects the boundary condition and we note that each of these solutions coincides with a function in $\mathfrak{D}\left(M_{1}\right)$ near its associated boundary point. This suggests that we consider $\mathfrak{D}\left(L_{1}\right)$ modulo

$$
\mathfrak{D}\left(L_{a}\right) \equiv\left[y ; y \in \mathfrak{D}\left(L_{1}\right),(A y, z)^{a}=0 \text { for all } z \in \mathfrak{D}\left(M_{1}\right)\right]
$$

in order to determine what is basic in the behavior of $y \in \mathfrak{D}\left(L_{1}\right)$ at $x=a$ and that we consider $\mathfrak{D}\left(L_{1}\right)$ modulo

$$
\mathfrak{D}\left(L_{b}\right) \equiv\left[y ; y \in \mathfrak{D}\left(L_{1}\right),(A y, z)^{b}=0 \text { for all } z \in \mathfrak{D}\left(M_{1}\right)\right]
$$

in order to determine the basic behavior of $y \in \mathfrak{D}\left(L_{1}\right)$ at $x=b$. It turns out that $(A y, y)^{a}$ is the same for all $y$ belonging to a coset of $\mathscr{D}\left(L_{1}\right) / \mathfrak{D}\left(L_{a}\right)$ and $(A y, y)^{b}$ is the same for all $y$ belong to a coset of $\mathfrak{D}\left(L_{1}\right) / \mathfrak{D}\left(L_{b}\right)$.

We shall show that the quotient space $\mathfrak{Y}_{b}=\mathfrak{D}\left(L_{1}\right) / \mathfrak{D}\left(L_{b}\right)$ [respectively $\left.\mathfrak{V}_{a}=\mathfrak{D}\left(L_{1}\right) / \mathfrak{D}\left(L_{a}\right)\right]$ is completely characterized by the solutions of (3.1) which lie in $L_{2}(c, b ; D)\left[L_{2}(a, c ; D)\right]$, that is, by the functions in $F_{b}\left[F_{a}\right]$. In fact, suppose for some $\lambda>0$ that $l_{b}\left[l_{a}\right]$ denotes the dimensionality of $F_{b}\left[F_{a}\right]$ and that $m_{b}\left[m_{a}\right]$ denotes the dimensionality of $G_{b}\left[G_{a}\right]$, that is, the set of solutions of $(3.2)$ which lie in $L_{2}(b, c ; D)\left[L_{2}(a, c ; D)\right]$. Then $\mathfrak{D}\left(L_{1}\right) / \mathfrak{D}\left(L_{b}\right)$ will be of dimension $d_{b}=l_{b}+m_{b}-r$ and, in fact, if we take into account only the behavior near $b$ of the functions in $F_{b}$, the corresponding cosets will span $\mathfrak{D}\left(L_{1}\right) / \mathfrak{D}\left(L_{b}\right)$, with an $r-m_{b}$ dimensional subspace of $F_{b}$ mapping into the zero coset. An analogous assertion holds at the $a$ end.

We begin by proving

TheOREM 5.1. $l_{a}, l_{b}, m_{a}$, and $m_{b}$ are independent of $\lambda>0$.

$c<x<b$. Hence $-\left(A u_{a, i}, z_{a}\right)^{a}+\left(A u_{b, i}, z_{b}\right)^{b}=0$ for all $\left[z_{a}, z_{b}\right] \in P_{a, b}\left(\lambda_{0}\right)$. By joining $u_{a, i}$ on $a<x<c$ and $u_{b, i}$ on $c<x<b$ smoothly near $x=c$, it is easily seen that the resulting function lies in $\mathfrak{D}(L)$ and it follows from this that $-\left(A u_{a}, u_{a}\right)^{a}+\left(A u_{b}, u_{b}\right)^{b} \leqq 0$ for all linear combinations $\left[u_{a}, u_{b}\right]$ of the $\left[u_{a, i}, u_{b, i}\right] ; i=1,2, \cdots, r$. Thus these solution pairs for (3.1) define an $r$-dimensional subspace $N_{a, b}(\lambda)$ of the type considered in Theorem 4.1 and we can obtain a dissipative operator $L_{\lambda}$ as before from the $A_{a, b}(\lambda)$-orthogonal complement $P_{a, b}(\lambda)$. Proceeding as in the proof of Theorem 4.1, we see that the range of $R\left(\lambda ; L_{\lambda}\right)$ is the closure in the graph topology of functions each of which behaves like $u_{a}$ near $x=a$ and like $u_{b}$ near $x=b$ where $\left[u_{a}, u_{b}\right] \in N_{a, b}(\lambda)$. Lemma 4.3 asserts that $-\left(A u, z_{a}\right)^{a}+\left(A u, z_{b}\right)^{b}=0$ for all $u \in D\left(L_{\lambda}\right)$ and all $\left[z_{a}, z_{b}\right] \in P_{a, b}\left(\lambda_{0}\right)$. Thus $L_{\lambda} \subset L$. Now starting with $L_{\lambda}$ we could just as well obtain a restriction $L_{\lambda_{0}} \subset L_{\lambda}$. However both $L_{\lambda_{0}}$ and $L$ have resolvents at $\lambda=\lambda_{0}$ and since $L_{\lambda_{0}} \subset L$ they must in fact be equal. Consequently $L_{\lambda_{0}}=L_{\lambda}=L$. Finally it is clear that any $\lambda>0$ can be reached from any $\lambda_{0}>0$ in a finite number of steps of the above kind and this proves that $L$ has a resolvent satisfying the HilleYosida condition for all $\lambda>0$. 
Proof. Let $l_{b}(\lambda)$ denote the dimension of $F_{b}(\lambda)$ for each $\lambda>0$ and suppose $\lambda, \lambda_{0}$ are such that $\left|\lambda-\lambda_{0}\right|<\lambda_{0}$. In this proof it will be convenient to restrict our attention to a fixed interval $(c, b)$ where $a<c<b$. It is clear that this will have no effect on the set $F_{b}(\lambda)$. Now, let $L$ be some dissipative restriction of $L_{1}$ defined as in Theorem 4.1 with the difference that the range of the independent variable is now $(c, b)$. Since $\left\|R\left(\lambda_{0}, L\right)\right\| \leqq \lambda_{0}^{-1}$, the integral equation

$$
u-\left(\lambda_{0}-\lambda\right) R\left(\lambda_{0} ; L\right) u=f, \quad f \in L_{2}(c, b ; I),
$$

has a unique solution which can be expressed, for instance, by a Neumann series. In particular, for $f=y \in F_{b}\left(\lambda_{0}\right)$ (defined on $(c, b)$ ), we obtain a solution $u$ with $A u$ absolutely continuous and satisfying

$$
\lambda u-(A u)_{x}-B u=\theta
$$

for almost all $x \in(c, b)$. Since the integral equation (5.3) expresses $u$ as the sum of an element in $\mathfrak{D}(L)$ plus $y$, we see that $u \in L_{2}(c, b ; D)$. Clearly $u$ can be extended to be a solution of $(3.1)$ on $(a, b)$ and the so extended function belongs to $F_{b}(\lambda)$. The uniqueness of the solution to (5.3) implies that any linearly independent set of $y$ 's in $F_{b}\left(\lambda_{0}\right)$ corresponds in this way to a linearly independent set of $u$ 's in $F_{b}(\lambda)$. Consequently $l_{b}(\lambda) \geqq l_{b}\left(\lambda_{0}\right)$. Now starting with a given $\lambda_{0}$ we can reach any $\lambda>0$ in a finite number of steps of the kind described above. It follows that $l_{b}(\lambda) \geqq l_{b}\left(\lambda_{0}\right)$ for all $\lambda>0$. This relation being symmetric, we see that $l_{l}(\lambda)$ is in fact independent of $\lambda$ for $\lambda>0$. A similar argument proves the analogous assertions for $l_{a}, m_{a}$, and $m_{b}$.

In a different direction we require the following lemma, due in essence to F. Rellich [15].

Lemma 5.1. Given the vector-valued functions $\left[w_{i} ; i \leqq t\right]$ defined on $(a, b)$ but such that for each compact subinterval $\left[a^{\prime}, b^{\prime}\right]$ of $(a, b)$ they define linearly independent elements of $L_{2}\left(a^{\prime}, b^{\prime} ; I\right)$. Assume further that the first $s$ of these belong to $H=L_{2}(a, b ; I)$. Finally let $S$ denote the set of all bounded measurable vectorvalued functions $f$ vanishing near $a$ and $b$, and such that

$$
\int_{a}^{b}\left(f, w_{i}\right) d x=0 \quad \text { for all } i \leqq t .
$$

Then $S$ is contained in and is dense in the orthogonal complement $J$ of $\left[w_{i} ; i \leqq s\right]$ in $H$ if and only if $\left[w_{i} ; i \leqq s\right]$ spans the largest subspace of the linear extension of $\left[w_{i} ; i \leqq t\right]$ which is contained in $H$, that is, if and only if $\sum_{i=1}^{t} \gamma_{i} w_{i} \in H$ implies that $\gamma_{i}=0$ for all $i>s$.

Proof. Suppose first that $S$ is contained in and dense in $J$, and that $w=\sum_{i=1}^{t} \gamma_{i} w_{i} \in H$. Since $w$ is orthogonal to $S$ by (5.4), we conclude that it has a representation of the form $w=\sum_{i=1}^{s} \gamma_{i}^{\prime} w_{i}$. On the other hand, the $w_{i}$ are assumed to be linearly independent on $\left[a^{\prime}, b^{\prime}\right]<(a, b)$ so that $\gamma_{i}=\gamma_{i}^{\prime}$ for $i \leqq s$ and $\gamma_{i}=0$ for $i>s$. 
Conversely, suppose that $\left[w_{i} ; i \leqq s\right]$ spans the largest subspace in the linear extension of $\left[w_{i} ; i \leqq t\right]$ which is contained in $H$. Since $S$ is obviously contained in $J$, it remains to show only that $S$ is dense in $J$. If this were not the case there would exist a nonzero $w \in J$ orthogonal to $S$. For each compact subinterval $\left[a^{\prime}, b^{\prime}\right] \subset(a, b)$, the functions of $S$ which vanish outside of $\left(a^{\prime}, b^{\prime}\right)$ are dense in the orthogonal complement of $\left[w_{i} ; i \leqq t\right]$ relative to $L_{2}\left(a^{\prime}, b^{\prime} ; I\right)$ and hence $w$ lies in the linear extension of the functions $\left[w_{i} ; i \leqq t\right]$ restricted to $\left(a^{\prime}, b^{\prime}\right)$. Thus $w=\sum_{i=1}^{t} \gamma_{i}^{\prime} w_{i}$ almost everywhere in $\left(a^{\prime}, b^{\prime}\right)$. Now the same argument applies to the interval $\left(a^{\prime \prime}, b^{\prime \prime}\right), a<a^{\prime \prime}<a^{\prime}<b^{\prime}<b^{\prime \prime}<b$, and gives $w=\sum_{i=1}^{t} \gamma_{i}^{\prime \prime} w_{i}$ almost everywhere in $\left(a^{\prime \prime}, b^{\prime \prime}\right)$. The linear independence of the $w_{i}$ on $\left(a^{\prime}, b^{\prime}\right)$ implies that $\gamma_{i}^{\prime}=\gamma_{i}^{\prime \prime}$ and it follows that $w=\sum_{i=1}^{t} \gamma_{i}^{\prime} w_{i}$ almost everywhere on $(a, b)$. Since $w \in H$, we see that $\gamma_{i}^{\prime}=0$ for $i>s$ so that $w$ lies in the orthogonal complement to $J$, contrary to our choice of $w$; we must therefore conclude that $S$ is actually dense in $J$.

We return once more to the question of limit theorems and prove

Lemma 5.2. If $\left\{y_{n} ; n=1,2, \cdots\right\} \subset \mathfrak{D}\left(L_{1}\right)$ and

$$
\left[y_{n}, L_{1} y_{n}\right] \rightarrow\left[y_{0}, h_{0}\right] \text { in } H^{2},
$$

then $y_{0} \in D\left(L_{1}\right), L_{1}\left(y_{0}\right)=h_{0}$, and in fact $y_{n} \rightarrow y_{0}$ in $L_{2}(a, b ; D)$.

Proof. Given $\lambda>0$, let $L$ be a dissipative restriction of $L_{1}$ defined as in Theorem 4.1 so that the resolvent $R(\lambda ; L)$ exists. Further let $\left[u_{i} ; i \leqq l\right]$ form a basis for the solutions of $\lambda u-L_{1} u=\theta$, that is, for the solutions of (3.1) which lie in $L_{2}(a, b ; D)$. Then for any $y \in \mathfrak{D}\left(L_{1}\right), f \equiv \lambda y-L_{1} y$, we can write the solution of this equation, namely $y$, as a particular solution in $\mathfrak{D}(L) \subset L_{2}(a, b ; D)$ is a solution of the homogeneous equation lying in $L_{2}(a, b ; D)$; thus

$$
y=R(\lambda ; L) f+\sum_{i=1}^{r} \gamma_{i}(y) u_{i} .
$$

We note that this representation is unique. Now if (5.5) holds, then $f_{n}$ $=\lambda y_{n}-L_{1} y_{n} \rightarrow \lambda y_{0}-h_{0} \equiv f_{0}$ in $H$ and hence by Lemma $4.5 R(\lambda ; L) f_{n} \rightarrow R(\lambda ; L) f_{0}$ in $L_{2}(a, b ; D)$. As a consequence $\sum \gamma_{i}\left(y_{n}\right) u_{i}$ converges to a limit, at least in $H$. But this implies that each of the sequences $\left\{\gamma_{i}\left(y_{n}\right) ; n=1,2, \cdots\right\}$ converges to a limit $\left(^{8}\right)$, say $\gamma_{i}$, so that $\sum \gamma_{i}\left(y_{n}\right) u_{i} \rightarrow \sum \gamma_{i} u_{i}$ also in $L_{2}(a, b ; D)$. This proves that $y_{n} \rightarrow y_{0}$ in $L_{2}(a, b ; D)$. Incidentally, we have shown that $y_{0}$ has the form (5.6), from which it follows that $y_{0} \in \mathfrak{D}\left(L_{1}\right)$ and that $L_{1} y_{0}=\lambda y_{0}-f_{0}$ $=h_{0}$.

Corollary. Let $Q_{a, b}$ be a given set of function pairs $\left[z_{a}, z_{b}\right]$ belonging to $\mathfrak{D}\left(M_{1}\right)$ and let $L$ be a restriction of $L_{1}$ with domain

$$
\mathfrak{D}(L)=\left[y ; y \in \mathfrak{D}\left(L_{1}\right),-\left(A y, z_{a}\right)^{a}+\left(A y, z_{b}\right)^{b}=0 \text { for all }\left[z_{a}, z_{b}\right] \in Q_{a, b}\right] .
$$

$\left.{ }^{8}\right)$ Since the $u_{i}$ 's are linearly independent, there exist vector-valued functions $\left\{w_{i} ; i \leqq l\right\}$ $C H$ such that $\left\langle u_{i}, w_{j}\right\rangle=\delta_{i j}$. Copsequently $\gamma_{j}\left(y_{n}\right)=\left\langle\sum_{i} \gamma_{i}\left(y_{n}\right) u_{i}, w_{j}\right\rangle$ converges to a limit. 
Then $L$ is a closed linear operator.

Proof. This follows directly from Lemmas 4.3 and 5.2.

We remark that the same type of argument as that used in the proof of Lemma 5.2 will show that $L_{1}^{\prime} \subset L_{\infty}$ with

$$
\mathfrak{D}\left(L_{1}^{\prime}\right)=\mathbf{I}\left[y ; y \in \mathfrak{D}\left(L_{\infty}\right), y \text { and } L_{\infty} y \in H\right]
$$

is also a closed operator. In this case we choose the $u_{i}$ 's to form a basis for the solutions of $\lambda y-L_{\infty} y=\theta$ which lie in $H$ rather than for those in $L_{2}(a, b ; D)$ as above.

We next prove the previously mentioned stronger form of Lemma 4.4.

LemMA 5.3. Suppose $\left\{y_{i, n} ; n=0,1,2, \cdots\right\} \subset \mathcal{D}\left(L_{\infty}\right) \cap L_{2}(a, b ; D)$, $i=1,2$, and that for each $i$

$$
\left[y_{i, n}, L_{\infty} y_{i, n}\right] \rightarrow\left[y_{i, 0}, L_{\infty} y_{i, 0}\right] \text { in } H^{2} \text { or in } L_{2}(a, b ; D) \times L_{2}\left(a, b ; D^{-1}\right) .
$$

Then

$$
\lim _{n \rightarrow \infty}\left(A y_{1, n}, y_{2, n}\right)^{x}=\left(A y_{1,0}, y_{2,0}\right)^{x} \text { for } x=a, b .
$$

Proof. We proceed as in the proof of Lemma 4.4. By Lemma 4.2, the sequences $\left\{u_{i, n} \equiv \beta y_{i, n}\right\}$ have the same convergence properties as the original sequences and vanish near $a$. Actually $\lim _{n} u_{i, n}=u_{i, 0}$ in $L_{2}(a, b ; D)$ under both sets of hypotheses, since this assertion follows from Lemma 5.2 when it is assumed that $\left[u_{i, n}, L_{\infty} u_{i, n}\right]$ converges in $H^{2}$. Writing

$$
\begin{aligned}
\left(A y_{1, n}, y_{2, n}\right)^{b}= & \left(A u_{1, n}, u_{2, n}\right)^{b}=\int_{a}^{b}\left[\left(L_{\infty} u_{1, n}, u_{2, n}\right)+\left(u_{1, n}, L_{\infty} u_{2, n}\right)\right] d x \\
& -\int_{a}^{b}\left(\left(B+B^{*}+A_{x}\right) u_{1, n}, u_{2, n}\right) d x,
\end{aligned}
$$

we see that both integrals converge to their $n=0$ counterparts. In fact, the first integral is an inner product relative to $H$ in the $H^{2}$ case and relative to the dual hilbert spaces $L_{2}(a, b ; D)$ and $L_{2}\left(a, b ; D^{-1}\right)$ in the other case. On the other hand, the second integral can also be thought of as an inner product and as such it is majorized by the $L_{2}(a, b ; D)$ inner product; since the sequences $\left\{u_{i, n}\right\}$ converge in $L_{2}(a, b ; D)$ in both cases, it follows that the second integral also converges.

We have a certain amount of freedom in our choice of solution pairs $\left[y_{a, i}, y_{b, i}\right]$ and $\left[z_{a, i}, z_{b, i}\right]$ which span $N_{a, b}$ and $P_{a, b}$ respectively and satisfy the relation (3.18). In fact, if we choose $N_{a, b}=N_{a} \times N_{b}$ and $P_{a, b}=P_{a} \times P_{b}$, as at the end of $\S 3$, we may first select an arbitrary basis of $y_{a, i}$ 's for $N_{a}$ and an arbitrary basis of $z_{a, i}$ 's for $P_{a}$ and thereafter choose the $y_{b, i}$ 's and the $z_{b, i}$ 's from $N_{b}$ and $P_{b}$, respectively, so as to satisfy (3.18). Now the subsets $N_{a}$ and $N_{b}$ have no nonzero solutions in common; in fact, $0 \leqq\left(A y_{a}, y_{a}\right)^{a}<\left(A y_{a}, y_{a}\right)^{b}$ 
for each nonzero element of $N_{a}$ and $0 \geqq\left(A y_{b}, y_{b}\right)^{b}$ for each nonzero element of $N_{b}$. Thus $N_{a}$ and $N_{b}$ together span the $r$-dimensional solution space of (3.1). Hence we can even choose the $y_{a, i}$ 's so that the first $l_{b}-n$ together with $N_{b}$ span $F_{b}$ and likewise we can choose the $z_{a, i}$ 's so that the first $m_{b}-p$ together with $P_{b}$ span $G_{b}$. We shall call such a choice of bases a canonical selection of solution pairs at $x=a$.

LEMMA 5.4. If $y_{0} \in \mathfrak{D}\left(L_{b}\right)$, then there exists a sequence $\left\{y_{n}\right\} \subset \mathfrak{D}\left(L_{1}\right)$ of functions vanishing near $b$ such that

$$
\left[y_{n}, L_{1} y_{n}\right] \rightarrow\left[y_{0}, L_{1} y_{0}\right] \text { in } L_{2}(a, b ; D) \times L_{2}\left(a, b ; D^{-1}\right) .
$$

An analogous result holds for $y_{0} \in \mathfrak{D}\left(L_{a}\right)$.

Proof. With $\beta$ defined as in Lemma 4.2, we set $u_{0}=\beta y_{0}$ and $w_{0}=(1-\beta) y_{0}$ so that $u_{0}$ vanishes near $x=a$ and $w_{0}$ vanishes near $x=b$. If we can approximate $u_{0}$ in the required manner by say $\left\{u_{n}\right\} \subset \mathfrak{D}\left(L_{1}\right)$, then the sequence $\left\{y_{n}=u_{n}+w_{0}\right\}$ will be a suitable approximating sequence for $y_{0}$. We may therefore assume, without loss of generality, that $y_{0}$ vanishes near $x=a$. In this case $y_{0}$ belongs to the domain of every dissipative restriction of $L_{1}$ defined as in Theorem 4.1. We shall in particular choose a restriction $L$ defined by a canonical selection of solution pairs at $x=a$. Setting $f_{0}=\lambda y_{0}-L y_{0}$ we have as in (3.24)

$$
\begin{aligned}
y_{0}=R(\lambda ; L) f_{0}=\left(\begin{array}{c}
R_{00}(\lambda) f_{0}^{0} \\
\theta
\end{array}\right) & -\sum_{i=1}^{p} y_{a, i} \int_{x}^{b}\left(f_{0}, z_{b, i}\right) d \xi \\
& -\sum_{i=p+1}^{r} y_{b, i} \int_{a}^{x}\left(f_{0}, z_{a, i}\right) d \xi .
\end{aligned}
$$

Now for arbitrary $y \in \mathfrak{D}\left(L_{1}\right)$ we have by (4.5)

$$
\int_{a}^{x}\left(\lambda y-L_{1} y, z_{a, i}\right) d \xi=-\left(A y, z_{a, i}\right)^{x}+\left(A y, z_{a, i}\right)^{a}
$$

since $\lambda z_{a}-M_{\infty} z_{a}=\theta$ almost everywhere. In particular for $y=y_{0} \in \mathfrak{D}\left(L_{b}\right)$ and vanishing near $x=a$ this becomes

$$
\int_{a}^{b}\left(f_{0}, z_{a, i}\right) d \xi=-\left(A y_{0}, z_{a, i}\right)^{b}+\left(A y_{0}, z_{a, i}\right)^{a}=0
$$

for $p<i \leqq m_{\iota}$, that is, for the $z_{a, i}$ 's which belong to $\mathfrak{D}\left(M_{1}\right)$. We now apply Lemma 5.1 with $w_{i}=D^{1 / 2} z_{a, i+p}, i=1,2, \cdots, n$, and $s=m_{b}-p$. By our choice of basis for $P_{a}$, the first $\left(m_{b}-p\right) w_{i}$ 's span the largest subspace of the linear extension of $\left[w_{i} ; i \leqq n\right]$ which lies in $H$. According to (5.9), the function $D^{-1 / 2} f_{0}$ belongs to the orthogonal complement in $H$ of $\left[w_{i} ; i \leqq m_{b}-p\right]$. The lemma asserts that $D^{-1 / 2} f_{0}$ can be approximated in $H$ by a sequence of 
bounded measurable functions $\left\{h_{m}\right\}$, which vanish near $a$ and $b$, and which are orthogonal to all of the $w_{i}$ 's. We note that $f_{n}=D^{1 / 2} h_{n}$ also belongs to $H$ since the elements of $D^{1 / 2}$ are majorized by the trace of $D$ and hence are square integrable on a suitable carrier of $h_{n}$. In addition, $f_{n} \rightarrow f_{0}$ in $L_{2}\left(a, b ; D^{-1}\right)$, the functions $f_{n}$ vanish near $a$ and $b$, and $f_{n}$ is orthogonal to all of the $z_{a, i}$. As a consequence, $y_{n} \equiv R(\lambda ; L) f_{n} \in \mathfrak{D}\left(L_{1}\right)$ vanishes near $b$, and by Lemma 4.5 $y_{n} \rightarrow y_{0}=R(\lambda ; L) f_{0}$ in $L_{2}(a, b ; D)$. This concludes the proof.

We introduce the restriction $L_{00} \subset L_{1}$ with domain

$$
\mathfrak{D}\left(L_{00}\right)=\left[y ; y \in \mathfrak{D}\left(L_{1}\right), y \text { vanishing near } a \text { and } b\right] .
$$

Since $L_{1}$ is closed, the closure of $L_{00}$, which we denote by $L_{0}$, is again a restriction of $L_{1}$. Obviously $\mathfrak{D}\left(L_{00}\right) \subset \mathfrak{D}\left(L_{a}\right) \cap \mathfrak{D}\left(L_{b}\right)$ and it follows from Lemma 4.3 that $\mathfrak{D}\left(L_{0}\right) \subset \mathfrak{D}\left(L_{a}\right) \cap \mathfrak{D}\left(L_{b}\right)$. In the other direction we have.

Corollary. In case $B+B^{*}+A_{x}=\Theta, \mathfrak{D}\left(L_{0}\right)=\mathfrak{D}\left(L_{a}\right) \cap \mathfrak{D}\left(L_{b}\right)$.

Proof. Suppose $y \in \mathfrak{D}\left(L_{a}\right) \cap \mathfrak{D}\left(L_{b}\right)$. Applying the previous lemma at each end separately, we obtain two sequences $\left\{y_{a, n}\right\}$ and $\left\{y_{b, n}\right\} \subset \mathcal{D}\left(L_{1}\right)$, each of which converges in the graph topology (here $D=I$ ) to $y$; the first sequence consisting of functions vanishing near $a$, and the second of functions vanishing near $b$. Let $\alpha$ and $\beta$ be defined as in Lemma 4.2. Then both $\alpha y_{a, n}$ and $\beta y_{b, n}$ vanish near $a$ and $b$ and in addition Lemma 4.2 asserts that

$$
\alpha y_{a, n}+\beta y_{b, n} \rightarrow \alpha y+\beta y=y
$$

again in the graph topology. Obviously $\alpha y_{a, n}+\beta y_{b, n} \in \mathfrak{D}\left(L_{00}\right)$ so that $y \in \mathfrak{D}\left(L_{0}\right)$. As a consequence $\mathfrak{D}\left(L_{0}\right) \supset \mathfrak{D}\left(L_{a}\right) \cap \mathfrak{D}\left(L_{b}\right)$, which was the only inequality in doubt.

Theorem 5.2. Let $\left\{y_{i}, i=1,2,3\right\} \subset \mathfrak{D}\left(L_{1}\right)$ and suppose $y_{1}-y_{2} \in \mathfrak{D}\left(L_{b}\right)$. Then $\left(A y_{1}, y_{3}\right)^{b}=\left(A y_{2}, y_{3}\right)^{b}$. A similar result holds at $x=a$.

Proof. Set $u=y_{1}-y_{2}$. Then $u \in \mathfrak{D}\left(L_{b}\right)$ and, applying the previous lemma, we obtain an approximating sequence $\left\{u_{n}\right\} \subset \mathcal{D}\left(L_{1}\right)$ of functions vanishing near $x=b$ such that

$$
\left[u_{n}, L_{1} u_{n}\right] \rightarrow\left[u, L_{1} u\right] \text { in } L_{2}(a, b ; D) \times L_{2}\left(a, b ; D^{-1}\right) .
$$

Consequently

$$
\left[y_{2}+u_{n}, L_{1}\left(y_{2}+u_{n}\right)\right] \rightarrow\left[y_{1}, L_{1} y_{1}\right] \text { in } L_{2}(a, b ; D) \times L_{2}\left(a, b ; D^{-1}\right)
$$

and by Lemma 5.3 we have

$$
\left(A y_{2}, y_{3}\right)^{b}=\left(A\left(y_{2}+u_{n}\right), y_{3}\right)^{b} \rightarrow\left(A y_{1}, y_{3}\right)^{b} .
$$

This proves the assertion for the $b$ end and the corresponding result for the $a$ end follows by a similar argument.

We shall denote a coset of $\mathfrak{V}_{b}=\mathfrak{D}\left(L_{1}\right) / \mathfrak{D}\left(L_{b}\right)$ by the symbol $Y_{b}$. Given 
two such cosets $Y_{b, 1}$ and $Y_{b, 2}$, it follows from Theorem 5.2 that the quantity $\left(A y_{1}, y_{2}\right)^{b}$ is independent of our choice of $y_{1} \in Y_{b, 1}$ and $y_{2} \in Y_{b, 2}$ and hence can be used to define $\left(A Y_{b, 1}, Y_{b, 2}\right)$ in a unique way. We also define $\left(A Z_{b, 1}, Z_{b, 2}\right)$ in a similar fashion for cosets $Z_{b, 1}, Z_{b, 2} \in \mathfrak{Z}_{b}=\mathfrak{D}\left(M_{1}\right) / \mathfrak{D}\left(M_{b}\right)$. Finally for $y_{1}$, $y_{2} \in Y_{b}$ and $z_{1}, z_{2} \in Z_{b}$ we have $\left(A y_{1}, z_{1}\right)^{b}=\left(A y_{2}, z_{1}\right)^{b}=\left(A y_{2}, z_{2}\right)^{b}$, which can therefore be used to define $\left(A Y_{b}, Z_{b}\right)$.

In order to relate the solutions of $(3.1)$ which lie in $L_{2}(a, b ; D)$ with the quotient space $\mathfrak{D}_{b}$ we require the

LEMMA 5.5. For a canonical selection of solution pairs at $x=a$ and for any $y \in \mathfrak{D}\left(L_{1}\right)$ and $z \in \mathfrak{D}\left(M_{1}\right)$ we have

$$
\begin{aligned}
\left(A y, z_{b, i}\right)^{b} & =0 & \text { for } l_{b}-n & <i \leqq p, \\
\left(A y_{b, i}, z\right)^{b} & =0 & \text { for } m_{b} & <i \leqq r .
\end{aligned}
$$

Proof. Given $y \in \mathfrak{D}\left(L_{1}\right)$, we set $f \equiv \lambda y-L_{1} y$ and write $y$ as a particular solution of this equation in $L_{2}(a, b ; D)$ plus a solution of the homogeneous equation in $L_{2}(a, b ; D)$. For this purpose we choose a restriction $L$ of $L_{1}$ defined by a canonical selection of solution pairs at $x=a$ in which case

$$
y=R(\lambda ; L) f+\sum_{i=1}^{l_{0}-n} \gamma_{i} y_{a, i}+\sum_{i=p+1}^{r} \gamma_{i} y_{b, i} ;
$$

here we have summed over a basis for the solutions of (3.1) which belong to $L_{2}(c, b ; D)$. As in (3.24) the $y^{1}$ component of this identity can be written as

$$
\begin{aligned}
y^{1}= & -\sum_{i=1}^{p} y_{a, i}^{1} \int_{x}^{b}\left(f, z_{b, i}\right) d \xi-\sum_{i=p+1}^{r} y_{b, i}^{1} \int_{a}^{x}\left(f, z_{a, i}\right) d \xi \\
& +\sum_{i=1}^{l,-n} \gamma_{i} y_{a, i}^{1}+\sum_{i=p+1}^{r} \gamma_{i} y_{b, i}^{1} ;
\end{aligned}
$$

and if we make use of the relation (5.8) this becomes

$$
\begin{aligned}
y^{1}= & {\left[-\sum_{i=1}^{p} y_{a, i}^{1}\left(A y, z_{b, i}\right)^{x}+\sum_{i=p+1}^{r} y_{b, i}^{1}\left(A y, z_{a, i}\right)^{x}\right] } \\
& +\sum_{i=1}^{l_{b}-n}\left\{\gamma_{i}+\left(A y, z_{b, i}\right)^{b}\right\} y_{a, i}^{1}+\sum_{i=p+1}^{r}\left\{\gamma_{i}-\left(A y, z_{a, i}\right)^{a}\right\} y_{b, i}^{1} \\
& +\sum_{i=l_{b}-n+1}^{p}\left(A y, z_{b, i}\right)^{b} y_{a, i}^{1} .
\end{aligned}
$$

From the matrix relation (3.18) we see that $Y(x) Z(x)^{*} A_{11}(x, x)=I$ and this gives the identity

$$
-\sum_{i=1}^{p} y_{a, i}^{1}(x)\left(A_{11} h^{1}, z_{b, i}^{1}\right)^{x}+\sum_{i=p+1}^{r} y_{b, i}^{1}(x)\left(A_{11} h^{1}, z_{a, i}^{1}\right)^{x}=h^{1}(x) .
$$


We substitute this into (5.13), replacing $h$ by $y$ and $\left(A_{11} y^{1}, z^{1}\right)$ by $(A y, z)$, and we see that the last three sums add up to $\theta$. The linear independence of the $y_{a, i}^{1}$ 's and the $y_{b, i}^{1}$ 's implies that $\left(A y, z_{b, i}\right)^{b}=0$ for $l_{b}-n<i \leqq p$. The second half of the assertion (5.10) follows in a similar fashion; in this case we make use of a restriction $M$ of $M_{1}$ also defined by a canonical selection of solution pairs at $x=a$.

The next lemma is somewhat extraneous to our present development. However we shall need the result in $\$ 7$ and, since it is similar to the previous lemma, we include it at this point.

Lemma 5.6. Let the solution pairs $\left[y_{a, i}, y_{b, i}\right]$ and $\left[z_{a, i}, z_{b, i}\right]$ be chosen as in the canonical form of $R_{2}(\lambda)$ given in (4.23). Then for any $y \in \mathfrak{D}\left(L_{1}\right)$ we have

$$
\begin{aligned}
& \left(A y, z_{a, i}\right)^{a}=0 \\
& \text { for } i \in s_{3} \text {, } \\
& \left(A y, z_{b, i}\right)^{b}=0 \\
& \text { for } i \in s_{2} \text {. }
\end{aligned}
$$

Proof. We have already noted in connection with (4.23) that the solutions $\left\{y_{a, i}+y_{b, i} ; i=1,2, \cdots, r\right\}$ are linearly independent and hence span the solution space of (3.1). By construction, no nontrivial linear combination of $\left\{y_{a, i} ; i \in s_{2}\right\}$ and $\left\{y_{b, i} ; i \in s_{3}\right\}$ belongs to $L_{2}(a, b ; D)$. Hence we can express $y \in \mathfrak{D}\left(L_{1}\right)$ as the sum of a particular solution of $f=\lambda y-L_{1} y$ lying in $L_{2}(a, b ; D)$ plus a linear combination of the $y_{a, i}$ 's in $s_{1} \cup s_{3}$ and the $y_{b, i}$ 's in $s_{1} \cup s_{2}$; in symbols,

$$
y=R_{2}(\lambda) f+\sum_{s_{1} \cup s_{3}} \gamma_{i} y_{a, i}+\sum_{s_{1} \cup \mho_{2}} \gamma_{i} y_{b, i} \cdot
$$

Making use of the explicit representation for $R_{2}(\lambda)$ given in (4.23) and the relation (5.8) this becomes

$$
\begin{aligned}
y^{1}= & {\left[-\sum_{s_{1} \cup s_{2}}\left(y_{a, i}^{1}+y_{b, i}^{1}\right)\left(A y, z_{b, i}\right)+\sum_{s_{1} \cup s_{3}}\left(y_{a, i}^{1}+y_{b, i}^{1}\right)\left(A y, z_{a, i}\right)^{x}\right] } \\
& +\sum_{s_{1} \cup_{s_{3}}} \gamma_{i}^{\prime} y_{a, i}^{1}+\sum_{s_{1} \cup_{s_{2}}} \delta_{i}^{\prime} y_{b, i}^{1} \\
& +\sum_{s_{2}}\left(A y, z_{b, i}\right)^{b} y_{a, i}^{1}-\sum_{s_{3}}\left(A y, z_{a, i}\right)^{a} y_{b, i}^{1} .
\end{aligned}
$$

Again we see from the matrix relation $Y(x) Z(x) * A_{11}(x, x)=I$ that the quantity in the brackets is equal to $y^{1}(x)$ so that the last three sums in the right member add up to $\theta^{1}$. Any such solution of (3.1) must be the trivial solution. We have therefore expressed

$$
\sum_{b_{2}}\left(A y, z_{b, i}\right)^{b} y_{a, i}-\sum_{i z}\left(A y, z_{a, i}\right)^{a} y_{b, i}
$$

as a linear combination of solutions in $L_{2}(a, b ; D)$ and this is possible only if (5.14) holds 
TheOREM 5.3. 29 $)_{b}$ and $Z_{b}$ are both of dimension $d_{b}=l_{b}+m_{b}-r$.

Proof. We begin by choosing a canonical selection of solution pairs at $x=a$. Let $\beta$ be defined as in Lemma 4.2 and set

$$
\begin{array}{ll}
u_{a, i}=\beta y_{a, i}, & u_{b, i}=\beta y_{b, i} ; \\
v_{a, i}=\beta z_{a, i}, & v_{b, i}=-\beta z_{b, i} .
\end{array}
$$

Then the $u_{a, i}, i \leqq l_{b}-n$, and the $u_{b, i}, p+1 \leqq i \leqq r$, belong to $\mathfrak{D}\left(L_{1}\right)$ and the $v_{a, i}, p+1 \leqq i \leqq m_{b}$, and the $v_{b, i}, i \leqq p$, belong to $\mathfrak{D}\left(M_{1}\right)$. Moreover we have by (3.18)

$$
\begin{aligned}
& \left(A u_{a, i}, v_{b, j}\right)^{b}=\delta_{i j}=\left(A u_{b, i}, v_{a, j}\right)^{b} \\
& \left(A u_{a, i}, v_{a, j}\right)^{b}=0=\left(A u_{b, i}, v_{b, j}\right)^{b}
\end{aligned}
$$

Since only the $u$ 's and $v$ 's in $\mathfrak{D}\left(L_{1}\right)$ and $\mathfrak{D}\left(M_{1}\right)$, respectively, are relevant to our present considerations, the top relations in (5.16) establish only the linear independence modulo $\mathfrak{D}\left(L_{b}\right)$ of the $u_{a, i}, i \leqq l_{b}-n$, and the $u_{b, i}, p+1 \leqq i \leqq m_{b}$. Thus there are at least $\left(l_{b}-n\right)+\left(m_{b}-p\right)=l_{b}+m_{b}-r$ functions $u$ which are linearly independent modulo $\mathfrak{D}\left(L_{b}\right)$. Now as Lemma 5.5 shows, the $u_{t, i}$ for $m_{b}<i \leqq r$ lie in $\mathfrak{D}\left(L_{b}\right)$ so that the $u_{a, i}$ 's and $u_{b, i}$ 's in $\mathfrak{D}\left(L_{1}\right)$ provide exactly $l_{b}+m_{b}-r$ linearly independent functions modulo $\mathfrak{D}\left(L_{b}\right)$.

On the other hand an arbitrary $y \in \mathfrak{D}\left(L_{1}\right)$ can be written in the form (5.11). Approximating $f=\lambda y-L_{1} y$ in $H$ by a sequence of functions $\left\{f_{n}\right\}$ vanishing near $x=b$ and setting

$$
y_{n}=R(\lambda ; L) f_{n}+\sum_{i=1}^{l_{b}-n} \gamma_{i} y_{a, i}+\sum_{i=p+1}^{r} \gamma_{i} y_{b, i} .
$$

We see that $\left[y_{n}, L_{1} y_{n}\right] \rightarrow\left[y, L_{1} y\right]$ in $H^{2}$. According to the representation (5.12) the $y_{n}^{1}$ 's behave like linear combinations of the $\left[y_{a, i}^{1} ; i \leqq l_{b}-n\right]$ and the $\left[y_{b, i}^{1} ; p+1 \leqq i \leqq r\right]$ for $x$ near $b$. Now for any $y \in \mathfrak{D}\left(L_{1}\right)$ and $z \in \mathfrak{D}\left(M_{1}\right)$ the quantity $(A y, z)^{b}$ depends only on the behavior of $y^{1}$ near $b$. Hence, modulo $\mathfrak{D}\left(L_{b}\right)$, the $y_{n}$ 's lie in the $\left(l_{b}+m_{b}-r\right)$-dimensional subspace determined by the $\left[u_{a, i} ; i \leqq l_{b}-n\right]$ and the $\left[u_{b, i} ; p+1 \leqq i \leqq m_{b}\right]$; in other words $y_{n}$ is equivalent modulo $\mathfrak{D}\left(L_{b}\right)$ to

$$
u_{n}=\sum_{i=1}^{l_{b}-n} \gamma_{n, i} u_{a, i}+\sum_{i=p+1}^{m_{b}} \gamma_{n, i} u_{b, i}
$$

It follows from (5.16) that

$$
\begin{array}{lr}
\gamma_{n, i}=\left(A u_{n}, v_{b, i}\right)^{b}=\left(A y_{n}, v_{b, i}\right)^{b}, & 1 \leqq i \leqq l_{b}-n, \\
\gamma_{n, i}=\left(A u_{n}, v_{a, i}\right)^{b}=\left(A y_{n}, v_{a, i}\right)^{b}, & p+1 \leqq i \leqq m_{b} .
\end{array}
$$

Lemma 4.3 asserts that $\left(A y_{n}, v\right)^{b} \rightarrow(A y, v)^{b}$ so that for 


$$
u=\sum_{i=1}^{l_{b}-n}\left(A y, v_{b, i}\right)^{b} u_{a, i}+\sum_{i=p+1}^{m b}\left(A y, v_{a, i}\right)^{b} u_{b, i}
$$

we have $\left[u_{n}, L_{1} u_{n}\right] \rightarrow\left[u, L_{1} u\right]$ in $H^{2}$. Again applying Lemma 4.3, we obtain

$$
(A y, z)^{b}=\lim _{n \rightarrow \infty}\left(A y_{n}, z\right)^{b}=\lim _{n \rightarrow \infty}\left(A u_{n}, z\right)^{b}=(A u, z)^{b} \text { for each } z \in \mathfrak{D}\left(M_{1}\right),
$$

and therefore $y$ is equivalent to $u$ modulo $\mathfrak{D}\left(L_{b}\right)$. Thus each $y \in \mathfrak{D}\left(L_{1}\right)$ lies modulo $\mathfrak{D}\left(L_{b}\right)$ in the $d_{b}$-dimensional subspace spanned by the $\left[u_{a, i} ; 1 \leqq i \leqq l_{b}-n\right]$ and the $\left[u_{b, i} ; p+1 \leqq i \leqq m_{b}\right]$. A similar argument shows that $\not_{b}$ is also of dimension $d_{b}$.

We have, incidentally, established the

Corollary. Let the $\left[y_{a, i}, y_{b, i}\right]$ and the $\left[z_{a}, z_{b, i}\right]$ be a canonical selection of solution pairs at $x=a$ and define the $\left[u_{a, i}, u_{b, i}\right]$ and the $\left[v_{a, i}, v_{b, i}\right]$ by (5.15). Then the cosets determined by the $\left[u_{a, i} ; i \leqq l_{b}-n\right]$ and the $\left[u_{b, i} ; p+1 \leqq i \leqq m_{b}\right]$ are linearly independent and span $\mathfrak{Y}_{b}$; likewise the cosets determined by the $\left[v_{a, i} ; p+1 \leqq i \leqq m_{b}\right]$ and the $\left[v_{b, i} ; i \leqq l_{b}-n\right]$ are linearly independent and span $\mathfrak{马}_{b}$. Moreover $\left[u_{b, i} ; m_{b}<i \leqq r\right] \subset \mathfrak{D}\left(L_{b}\right)$ and $\left[v_{b, i} ; l_{b}-n<i \leqq p\right] \subset \mathfrak{D}\left(M_{b}\right)$.

We can summarize the preceding development as follows. Let $\lambda>0$ be fixed and choose $\left[u_{a, i}, u_{b, i}\right]$ and $\left[v_{a, i}, v_{b, i}\right]$ as in (5.15) from a canonical selection of solution pairs at $x=a$. For notational convenience we set

$$
\begin{aligned}
& u_{i}=u_{a, i}, \quad v_{i}=v_{b, i}, \quad \text { for } i \leqq l_{b}-n, \\
& u_{i}=u_{b, r-l_{b}+i}, \quad v_{i}=v_{a, r-l_{b}+i} \quad \text { for } l_{b}-n<i \leqq d_{b} .
\end{aligned}
$$

Then $\left(A u_{i}, v_{j}\right)^{b}=\delta_{i, j}$ and for each $y \in \mathfrak{D}\left(L_{1}\right)$ and $z \in \mathfrak{D}\left(M_{1}\right)$ we have

$$
\begin{aligned}
& y \equiv \sum_{i=1}^{d_{b}}\left(A y, v_{i}\right)^{b} u_{i} \text { modulo } \mathfrak{D}\left(L_{b}\right), \\
& z \equiv \sum_{i=1}^{d_{b}}\left(A z, u_{i}\right)^{b} v_{i} \text { modulo } \mathfrak{D}\left(M_{b}\right) ;
\end{aligned}
$$

in fact, the first assertion follows directly from (5.17) and (5.18) above and the second assertion is the dual of the first. Moreover, Theorem 5.2 shows that for arbitrary $y_{1}, y_{2} \in \mathfrak{D}\left(L_{1}\right)$ and $z_{1}, z_{2} \in \mathfrak{D}\left(M_{1}\right)$ we have

$$
\begin{aligned}
& \left(A y_{1}, y_{2}\right)^{b}=\sum_{i, j=1}^{d_{b}}\left(A y_{1}, v_{i}\right)^{b}\left[\left(A y_{2}, v_{j}\right)^{b}\right]^{-}\left(A u_{i}, u_{i}\right)^{b}, \\
& \left(A y_{1}, z_{1}\right)^{b}=\sum_{i=1}^{d_{b}}\left(A y_{1}, v_{i}\right)^{b}\left[\left(A z, u_{i}\right)^{b}\right]^{-}, \\
& \left(A z_{1}, z_{2}\right)^{b}=\sum_{i, j=1}^{d_{b}}\left(A z_{1}, u_{i}\right)^{b}\left[\left(A z_{2}, u_{i}\right)^{b}\right]^{-}\left(A v_{i}, v_{j}\right)^{b} .
\end{aligned}
$$


In essence we have reduced the study of the boundary behavior to an algebraic problem involving certain finite dimensional quotient spaces. According to (5.21) the spaces $\mathfrak{Y}_{b}$ and $\mathfrak{B}_{b}$ are dual to one another and the matrix $A(b)$ has the representation $\left(\left(A u_{i}, u_{j}\right)^{b}\right)$ in one space and $\left(\left(A v_{i}, v_{j}\right)^{b}\right)$ in the other. In order to again formulate our boundary conditions as dual relations we require the connection between these two representations of $A(b)$.

Theorem 5.4. $\left(\left(A u_{i}, u_{j}\right)^{b}\right)\left(\left(A v_{i}, v_{j}\right)^{b}\right)=I$.

Proof. For an arbitrary $z \in \mathfrak{D}\left(M_{1}\right)$ it is clear that $z \in \mathfrak{D}\left(L_{\infty}\right) \cap L_{2}(a, b ; D)$ and

$$
L_{\infty} z=-M_{1} z+\left(B+B^{*}+A_{x}\right) z .
$$

Since the first term on the right belongs to $H$ and the second $\left({ }^{9}\right)$ to $L_{2}\left(a, b ; D^{-1}\right)$, it follows that $L_{\infty} z \in L_{2}\left(a, b ; D^{-1}\right)$. Consequently $f=\lambda z-L_{\infty} z \in L_{2}\left(a, b ; D^{-1}\right)$. Choosing a canonical selection of solution pairs at $x=a$ we set $w=R(\lambda) f$, where $R(\lambda)$ is of the form (3.24). Here all of the terms are well defined since the $z_{a}$ 's belong to $L_{2}(a, c ; D)$ and the $z_{b}$ 's belong to $L_{2}(c, b ; D)$ and, in fact, we see that $w \in \mathfrak{D}\left(L_{\infty}\right)$. We now show that $w$ also belongs to $L_{2}(a, b ; D)$. In the first place $f^{0}=\left(\lambda z^{0}-B_{00} z^{0}\right)-B_{01} z^{1}$ so that $R_{00}(\lambda) f^{0}=z^{0}-R_{00}(\lambda) B_{01} z^{1}$, and, $z^{1}$ being absolutely continuous, we see that $B_{01} z^{1}$ belongs to $L_{2}\left(a^{\prime}, b^{\prime} ; I_{00}\right)$ for each compact $\left[a^{\prime}, b^{\prime}\right] \subset(a, b)$. Now

$$
\begin{aligned}
\left(\left\{2 \lambda I_{00}-\left(B_{00}+B_{00}^{*}\right)\right\} R_{00}(\lambda) B_{01} z^{1},\right. & \left.R_{00}(\lambda) B_{01} z^{1}\right) \\
& =\left(B_{01} z^{1}, R_{00}(\lambda) B_{01} z^{1}\right)+\left(R_{00}(\lambda) B_{01} z^{1}, B_{01} z^{1}\right) .
\end{aligned}
$$

Recalling that $\lambda\left|R_{00}(\lambda)\right| \leqq 1$, it is clear that the displayed expression is summable on $\left(a^{\prime}, b^{\prime}\right)$ and it follows that $R_{00}(\lambda) f^{0}$ belongs to $L_{2}\left(a^{\prime}, b^{\prime} ; D_{00}\right)$. Since the rest of the terms in (3.24) obviously belong to $L_{2}\left(a^{\prime}, b^{\prime} ; D\right)$, the same is true of $w$. Further $\lambda w-L_{\infty} w=f \in L_{2}\left(a, b ; D^{-1}\right)$. Now if $f$ vanishes near $a$ and $b$, then by the usual argument $-(A w, w)^{a}+(A w, w)^{b} \leqq 0$ (see proof of Theorem 4.1) and hence $w$ belongs to $L_{2}(a, b ; D)$ by Lemma 4.5. If $f$ does not vanish near $a$ and $b$, we approximate $f$ in $L_{2}\left(a, b ; D^{-1}\right)$ by a sequence $\left\{f_{n}\right\}$ of bounded measurable vector-valued functions which do vanish near $a$ and $b$. Applying Lemma 4.5 , we see that the corresponding $w_{n}$ 's converge in $L_{2}(a, b ; D)$ to $w$ and hence $w \in L_{2}(a, b ; D)$. Thus $w$ is a particular solution of $\lambda u-L_{\infty} w=f$ in $L_{2}(a, b ; D)$ and therefore $z$ itself can be expressed as

$$
z=w+\sum_{i=1}^{l_{b}-n} \gamma_{i} y_{a, i}+\sum_{i=p+1}^{r} \gamma_{i} y_{b, i}
$$

for some choice of $\gamma_{i}^{\prime}$ 's; here each of the three expressions on the right lies in

(9) By definition $B+B^{*}+A_{x}=I-D$ so that $\left(D^{-1}\left(B+B^{*}+A_{x}\right) z,\left(B+B^{*}+A_{x}\right) z\right)=\left(D^{-1} z, z\right)$ $-2(z, z)+(D z, z)$; since all of the terms in the right member are integrable on $(a, b)$, it follows that $\left(B+B^{*}+A_{x}\right) z \in L_{2}\left(a, b ; D^{-1}\right)$. 
$L_{2}(a, b ; D)$. Setting

$$
z_{n}=w_{n}+\sum_{i=1}^{l_{b}-n} \gamma_{i} y_{a, i}+\sum_{i=p+1}^{r} \gamma_{i} y_{b, i}
$$

we see that $\left[z_{n}, L_{\infty} z_{n}\right] \rightarrow\left[z, L_{\infty} z\right]$ in $L_{2}(a, b ; D) \times L_{2}\left(a, b ; D^{-1}\right)$. By construction $f_{n} \in H$ so that $z_{n} \in \mathfrak{D}\left(L_{1}\right)$, and making use of the first relation in (5.21) we can write

$$
\left(A z_{n}, u_{k}\right)^{b}=\sum_{j=1}^{d_{b}}\left(A z_{n}, v_{j}\right)^{b}\left(A u_{j}, u_{k}\right)^{b}
$$

Passing to the limit as $n \rightarrow \infty$, we obtain

$$
\left(A z, u_{k}\right)^{b}=\sum_{j=1}^{d_{b}}\left(A z, v_{j}\right)^{b}\left(A u_{j}, u_{k}\right)^{b}, \quad z \in \mathfrak{D}\left(M_{1}\right),
$$

the limit procedures being justified by Lemma 5.3 for the left member and by Lemma 4.3 for the right member. Finally setting $z=v_{i}$ we have

$$
\delta_{i k}=\left(A v_{i}, u_{k}\right)^{b}=\sum_{j=1}^{d_{b}}\left(A v_{i}, v_{j}\right)^{b}\left(A u_{j}, u_{k}\right)^{b}
$$

which was to be proved.

THEOREM 5.5. Each of the hermetian matrices $\left(\left(A u_{i}, u_{j}\right)^{b}\right)$ and $\left(\left(A v_{i}, v_{j}\right)^{b}\right)$ has $l_{b}-n$ positive eigenvalues and $m_{b}-p$ negative eigenvalues.

Proof. It is clear that the matrices are hermitian since $A(x)$ has this property for each $x$. Further, if $y_{a}$ is nontrivial element of $N_{a}$ and $x>a$, then $0 \leqq\left(A y_{a}, y_{a}\right)^{a}<\left(A y_{a}, y_{a}\right)^{x}$. Consequently we will have $\left(A u_{a}, u_{a}\right)^{b}>0$ for any nontrivial linear combination $u_{a}$ of the $u_{a, i}$ 's in $L_{2}(a, b ; D)$. The $u_{a, i}$ 's in $L_{2}(a, b ; D)$ constitute the first $l_{b}-n$ of the $u_{i}$ 's and hence there are at least $l_{b}-n$ positive eigenvalues for $\left(\left(A u_{i}, u_{j}\right)^{b}\right)$. Next, if $y_{b} \in N_{b}$ then $\left(A y_{b}, y_{b}\right)^{b} \leqq 0$ and thus for any linear combination $u_{b}$ of the $u_{b, i}$ 's we have $\left(A u_{b}, u_{b}\right)^{b} \leqq 0$. Since the last $m_{b}-p$ of the $u_{i}$ 's are taken from the $u_{b, i}$ 's, the matrix $\left(\left(A u_{i}, u_{j}\right)^{b}\right)$ has at least $m_{b}-p$ nonpositive eigenvalues. On the other hand this matrix is nonsingular, according to Theorem 5.4, so that there can be no zero eigenvalues, and this, together with the fact that $d_{b}=\left(l_{b}-n\right)+\left(m_{b}-p\right)$, shows that there are precisely $l_{b}-n$ positive and $m_{b}-p$ negative eigenvalues. The same assertion obviously holds for the inverse matrix $\left(\left(A v_{i}, v_{j}\right)^{b}\right)$.

We have already defined the quantities $\left(A Y_{b, 1}, Y_{b, 2}\right),\left(A Y_{b}, Z_{b}\right),\left(A Z_{b, 1}, Z_{b, 2}\right)$ for the cosets $Y_{b} \in \bigvee_{b}$ and $Z_{b} \in Z_{b}$. Suppose, now that we choose as a basis for $\bigvee_{b}$ the set $\left[U_{b, i} ; i \leqq d_{b}\right]$ determined by the $u_{i}$ 's of $(5.19)$ and as a basis for $Z_{b}$ the set $\left[V_{b, i} ; i \leqq d_{b}\right]$ determined by the $v_{i}$ 's of (5.19). Then (5.20) and (5.21) can be paraphrased as 


$$
\begin{aligned}
Y_{b} & =\sum_{i=1}^{d_{b}}\left(A Y_{b}, V_{b, i}\right) U_{b, i}, \\
Z_{b} & =\sum_{i=1}^{d_{b}}\left(A Z_{b}, U_{b, i}\right) V_{b, i},
\end{aligned}
$$

and

$$
\begin{aligned}
\left(A Y_{b, 1}, Y_{b, 2}\right) & =\sum_{i, j=1}^{d_{b}}\left(A Y_{b, 1}, V_{b, i}\right)\left(A Y_{b, 2}, V_{b, j}\right)^{-}\left(A U_{b, i}, U_{b, j}\right), \\
\left(A Y_{b}, Z_{b}\right) & =\sum_{i=1}^{d_{b}}\left(A Y_{b}, V_{b, i}\right)\left(A Z_{b}, U_{b, i}\right)^{-}, \\
\left(A Z_{b, 1}, Z_{b, 2}\right) & =\sum_{i, j=1}^{d_{b}}\left(A Z_{b, 1}, U_{b, 1}\right)\left(A Z_{b, 2}, U_{b, j}\right)^{-}\left(A V_{b, i}, V_{b, j}\right) .
\end{aligned}
$$

The matrices $\left(\left(A U_{b, i}, U_{b, j}\right)\right)$ and $\left(\left(A V_{b, i}, V_{b, j}\right)\right)$ are each representations of the hermitian operator $A(b)$ relative to the respective bases, and by Theorem 5.4 there are inverses of one another. According to Theorem 5.5, $A(b)$ has $l_{b}-n$ positive and $m_{b}-p$ negative eigenvalues. We recall that the above choice of bases depended on a particular canonical selection of solution pairs, with given $\lambda>0$, at $x=a$. A different choice of canonical selection of solution pairs at $x=a$ and/or a different $\lambda>0$ would result in a coordinate transformation leaving the form of the relations (5.24) invariant.

Before leaving this subject we shall make the following convention. We shall speak of a function $y(x)$ as mapping into a coset of $\mathfrak{Y}_{b}$ even in case $y$ does not belong to $\mathfrak{I}\left(L_{1}\right)$ provided $y$ behaves like some function in $\mathfrak{D}\left(L_{1}\right)$ near $x=b$. In this case $u=\beta y$ will belong to $I\left(L_{1}\right)$ for an appropriate $\beta$ of the type described in Lemma 4.2 , and we map $y$ into the coset determined by $u$. This coset is clearly independent of the choice of $\beta$. Moreover since the limit behavior of $y$ at the $b$ end depends only on how it is defined near $x=b$, this information will again be given by the image coset of $y$.

It is clear that the boundary behavior at the $a$ end can be treated in a similar fashion. The quotient spaces $\mathfrak{Y}_{a}=\mathfrak{D}\left(L_{1}\right) / \mathfrak{D}\left(L_{a}\right)$ and $\mathfrak{Z}_{a}=\mathfrak{D}\left(M_{1}\right) / \mathfrak{D}\left(M_{a}\right)$ will each be a dimension $d_{a}=l_{a}+m_{a}-r$, we can construct bases for these spaces from a canonical selection of solution pairs at $x=b$ in terms of which analogues of (5.23) and (5.24) are valid, the representations $\left(\left(A U_{a, i}, U_{a, j}\right)\right)$ and $\left(\left(A V_{a, i}, V_{a, j}\right)\right)$ of $A(a)$ are again inverses of one another, and $A(a)$ will have $m_{a}-n$ positive and $l_{a}-p$ negative eigenvalues.

6. The general dissipative generator. We return now to the problem of constructing the most general dissipative restriction $L$ of $L_{1}$ generating a strongly continuous semi-group of linear bounded operators.

Our first objective is to formulate the boundary conditions on $L$ in a way which does not depend on $\lambda$. To this end we introduce the $d=d_{a}+d_{b}$ dimensional product spaces. 


$$
\begin{aligned}
& \mathfrak{Y}_{a, b}=\mathfrak{Y}_{a} \times \mathfrak{Y}_{b}, \\
& 3_{a, b}=\mathfrak{Z}_{a} \times \mathfrak{Z}_{b}
\end{aligned}
$$

with elements $Y_{a, b}=\left[Y_{a}, Y_{b}\right]$ and $Z_{a, b}=\left[Z_{a}, Z_{b}\right]$, respectively; and we define the operator $\mathfrak{R}_{a, b}$ by

$$
\begin{aligned}
\left(\mathfrak{H}_{a, b} Y_{a, b ; 1}, Y_{a, b, 2}\right) & =-\left(A Y_{a, 1}, Y_{a, 2}\right)+\left(A Y_{b, 1}, Y_{b, 2}\right), \\
\left(\mathfrak{A}_{a, b} Y_{a, b}, Z_{a, b}\right) & =-\left(A Y_{a}, Z_{a}\right)+\left(A Y_{b}, Z_{b}\right), \\
\left(\mathfrak{H}_{a, b} Z_{a, b, 1}, Z_{a, b ; 2}\right) & =-\left(A Z_{a, 1}, Z_{a, 2}\right)+\left(A Z_{b, 1}, Z_{b, 2}\right) .
\end{aligned}
$$

Relative to the canonical bases for $\mathfrak{Y}_{a, b}$ and $\mathfrak{Z}_{a, b}$ obtained in $\$ 5$, the operator $\mathfrak{A}_{a, b}$ has the representations

$$
\begin{aligned}
\mathfrak{A}_{a, b} & =\left(\begin{array}{cc}
-\left(\left(A U_{a, i}, U_{a, j}\right)\right) & 0 \\
0 & \left(\left(A U_{l, i}, U_{b, j}\right)\right)
\end{array}\right) \\
\Gamma \mathfrak{R}_{a, b}^{-1} \Gamma=\left(\begin{array}{cc}
-\left(\left(A V_{a, i}, V_{a, j}\right)\right) & 0 \\
0 & \left(\left(A V_{b, i}, V_{b, j}\right)\right)
\end{array}\right) & \text { in } \mathfrak{Y}_{a, b},
\end{aligned}
$$

where

$$
\Gamma=\left(\begin{array}{rr}
-I & 0 \\
0 & I
\end{array}\right)
$$

is a representation of the connective inner product between the bases given by ( $\left.\mathfrak{P}_{a, b} Y_{a, b}, Z_{a, b}\right)$. According to Theorems 5.4 and 5.5 , the matrix $\mathfrak{A}_{a, b}$ is nonsingular and hermitian, having $\left(l_{a}-p\right)+\left(l_{b}-n\right)=l_{a}+l_{b}-r$ positive and $\left(m_{a}-n\right)+\left(m_{b}-p\right)=m_{a}+m_{b}-r$ negative eigenvalues.

We now select an $\left(m_{a}+m_{b}-r\right)$-dimensional subspace $\mathfrak{R}_{a, b}$ of $\mathfrak{Y}_{a, b}$ such that

$$
\left(\mathfrak{A}_{a, b} Y_{a, b}, Y_{a, b}\right) \leqq 0
$$

for all $Y_{a, b} \in \mathfrak{N}_{a, b}$

and let $\mathfrak{B}_{a, b}$ denote the $\mathfrak{P}_{a, b}$-orthogonal complement of $\mathfrak{N}_{a, b}$ in $\mathfrak{Z}_{a, b}$, that is,

$$
\mathfrak{P}_{a, b}=\left[Z_{a, b} ;\left(\mathfrak{P}_{a, b} Y_{a, b}, Z_{a, b}\right)=0 \text { for all } Y_{a, b} \in \mathfrak{N}_{a, b}\right] .
$$

According to the corollary to Lemma 3.2, $P_{a, b}$ will be $\left(l_{a}+l_{b}-r\right)$ dimensional,

$$
\left(\mathfrak{H}_{a, b} Z_{a, b}, Z_{a, b}\right) \geqq 0 \quad \text { for all } Z_{a, b} \in \mathfrak{P}_{a, b},
$$

and $\mathfrak{N}_{a, b}$ is the $\mathfrak{A}_{a, b}$-orthogonal complement of $P_{a, b}$ in $\mathfrak{V}_{a, b}$.

ThEOREM 6.1. Suppose $\mathfrak{N}_{a, b}$ and $\mathfrak{P}_{a, b}$ are $\mathfrak{A}_{a, b}$-orthogonal complements in $\mathfrak{Y}_{a, b}$ and $\aleph_{a, b}$, respectively, satisfying the conditions (6.2) and (6.4). Let $L$ and $M$ be restrictions of $L_{1}$ and $M_{1}$, respectively, with domains

$$
\begin{aligned}
\mathfrak{D}(L) & =\left[y ; y \in \mathfrak{D}\left(L_{1}\right),[y, y] \rightarrow \mathfrak{N}_{a, b}\right], \\
\mathfrak{D}(M) & =\left[z ; z \in \mathfrak{I}\left(M_{1}\right),[z, z] \rightarrow \mathfrak{P}_{a, b}\right] .
\end{aligned}
$$

Then $L$ and $M$ are dissipative operators satisfying the Hille-Yosida criterion for 
all $\lambda>0$. Moreover $L=M^{*}$ and $M=L^{*}$.

REMARK. For a given $y \in \mathfrak{D}\left(L_{1}\right)$ we have $[y, y] \rightarrow \mathfrak{P}_{a, b}$ if and only if $-\left(A y, z_{a}\right)^{a}+\left(A y, z_{b}\right)^{b}=0$ for all $\left[z_{a}, z_{b}\right] \in \mathfrak{I}\left(M_{1}\right) \times \mathfrak{D}\left(M_{1}\right)$ which map into $\mathfrak{P}_{a, b}$. Hence (6.5) is equivalent with

$$
\mathfrak{D}(L)=\left[y ; y \in \mathfrak{D}\left(L_{1}\right),-\left(.1 y, z_{a}\right)^{a}+\left(A y, z_{b}\right)=0 \text { for all }\left[z_{a}, z_{b}\right] \rightarrow \mathfrak{P}_{a, b}\right],
$$

$\mathfrak{D}(M)=\left[z ; z \in \mathfrak{D}\left(M_{1}\right),-\left(A y_{a}, z\right)^{a}+\left(A y_{b}, z\right)^{b}=0\right.$ for all $\left.\left[y_{a}, y_{b}\right] \rightarrow \mathfrak{R}_{a, b}\right]$.

Proof. Suppose $\lambda>0$ is fixed. Let $N_{a, b}$ denote the solution pairs of (3.1) which map into $\mathfrak{R}_{a, b}$ and let $P_{a, b}$ denote the solution pairs of (3.2) which map into $\mathfrak{P}_{a, b}$. Taking into account the fact that $r-m_{a}$ linearly independent solutions of (3.1) map into $\mathfrak{D}\left(L_{a}\right)$ and $r-m_{b}$ map into $\mathfrak{D}\left(L_{b}\right)$, we see that $N_{a, b}$ is $\left(m_{a}+m_{b}-r\right)+\left(r-m_{a}\right)+\left(r-m_{b}\right)=r$ dimensional. Similarly $P_{a, b}$ is $r$ dimensional. Since the boundary behavior of these solution pairs is given by the cosets determined by them, the sets $N_{a, b}$ and $P_{a, b}$ will have all of the properties of the corresponding sets defined in Theorem 4.1. Thus $P_{a, b}$ is $r$-dimensional and satisfies (4.16); $N_{a, b}$ is $r$-dimensional and $A_{a, b}$-orthogonal to $P_{a, k}$, and since there is only one $r$-dimensional subspace of solution pairs with this property, $N_{a, b}$ must indeed be equal to the set defined by (4.18). According to the corollary to Theorem 5.3, the solutions of (3.2) which belong to $L_{2}(c, b ; D)$ (respectively $L_{2}(a, c ; D)$ ) span $\mathfrak{D}\left(M_{1}\right) / \mathfrak{D}\left(M_{b}\right)$ (respectively $\left.\mathfrak{D}\left(M_{1}\right) / \mathfrak{D}\left(M_{a}\right)\right)$. It follows that the solution pairs of (3.2) span $\mathfrak{Z}_{a, b}$ and this implies that the domain defined by (4.17) is the same as that defined for $L$ by (6.6) and hence by (6.5). Likewise $\mathfrak{D}(M)$ is equivalently defined by (4.24) and (6.5). The assertions of the theorem are now immediate consequences of Theorems 4.1 and 4.2 .

We also have a converse statement.

THEOREM 6.2. Every dissipative restriction of $L_{1}$ (or of $M_{1}$ ) which generates a strongly continuous semi-group of linear bounded operators is of the type described in Theorem 6.1.

Proof. Suppose $L^{0}$ is a dissipative restriction of $L_{1}$ which generates a semi-group. Then for each $y \in \mathfrak{I}\left(L^{0}\right)$ we have $-(A y, y)^{a}+(A y, y)^{b} \leqq 0$. Thus $\left[[y, y] ; y \in \mathfrak{D}\left(L^{0}\right)\right]$ maps onto a certain linear subspace $\mathfrak{\Re}_{a, b}^{0}$ of $\mathfrak{Y}_{a, b}$. Now $\mathfrak{N}_{a, b}^{0}$ cannot be more than $\left(m_{a}+m_{b}-r\right)$ dimensional since no subspace of larger dimension has the dissipative property. On the other hand there always exists an $\left(m_{a}+m_{b}-r\right)$-dimensional subspace $\mathfrak{N}_{a, b}$ of $\mathfrak{Y}_{a, b}$ containing $\mathfrak{N}_{a, b}^{0}$, with property (6.2), and the operator $L$ defined as in Theorem 6.1 from $\mathfrak{N}_{a, b}$ is clearly a dissipative extension of $L^{0}$. Now both $L^{0}$ and $L$ generate semi-groups and hence both have resolvents for $\lambda$ sufficiently large. Thus for some $\lambda$ the operators $\lambda I-L$ and $\lambda I-L^{0}$ map their respective domains onto all of $H$ in a 1-1 manner and this can only happen if $\mathfrak{I}(L)=\mathfrak{T}\left(L^{0}\right)$. Thus $L^{0}$ coincides with $L$ 
and is therefore of the type described in Theorem 6.1.

7. Coupled systems. In order to treat dissipative systems with boundary conditions of the "elastic" type, it is necessary to couple the previously considered system at the ends $a$ and $b$ with simple mechanical systems. The development which we are about to present for the coupled system parallels that which we have given for the uncoupled systems. In particular, we shall again take as the basic entity the energy of the system.

We consider vector functions $\mathfrak{y}=\left(\mathfrak{y}^{1}, \mathfrak{y}^{0}, \mathfrak{y}^{2}\right)$ and $z^{2}=\left(z^{1}, z^{0}, z^{2}\right)$ where $\mathfrak{y}^{1}, z^{1}$ lie in a $k_{1}$-dimensional euclidean space $\mathfrak{V}_{1}=\mathfrak{Z}_{1} ; \mathfrak{y}^{0}, \mathfrak{z}^{0} \in L_{2}\left(a, b ; \mathfrak{E}_{00}\right)$, and $\mathfrak{y}^{2}, z^{2}$ lie in a $k_{2}$-dimensional euclidean space $\mathfrak{Y}_{2}=Z_{2}$. In addition to the differential system (1.1) (with $E, A, B$ replaced by $\mathfrak{E}_{00}, \mathfrak{A}_{00}, \mathfrak{B}_{00}$ respectively) which governs $\mathfrak{y}^{0}$, we now have mechanical end systems which satisfy $\left({ }^{10}\right)$

$$
\begin{aligned}
& \mathcal{E}_{11 \mathfrak{1}} \eta_{\mathfrak{t}}^{1}=\mathfrak{B}_{11} \mathfrak{\eta}^{1}+\mathfrak{B}_{10} \mathfrak{\eta}^{0}(a), \\
& \left(\xi_{22} \eta_{t}^{2}=\mathfrak{B}_{22} \eta^{2}+\mathfrak{B}_{20} \eta^{0}(b)\right. \text {; }
\end{aligned}
$$

here the $\mathcal{E}_{i i}$ are positive definite,

$$
\mathfrak{B}_{i i}+\mathfrak{B}_{i i}^{*} \leqq \Theta,
$$$$
i=1,2,
$$

and $\mathfrak{B}_{10}, \mathfrak{B}_{20}$ are coupling operators defined as linear transformations on $\mathfrak{V}_{a}$ to $\mathfrak{Z}_{1}$ and on $\mathfrak{V}_{b}$ to $\mathfrak{Y}_{2}$, respectively. The energy integral for the coupled system is

$$
\text { Energy }=\frac{1}{2}\left[\left(\mathfrak{F}_{11} \mathfrak{y}^{1}, \mathfrak{y}^{1}\right)+\int_{a}^{b}\left(\xi_{00} \mathfrak{y}^{0}, \mathfrak{y}^{0}\right) d x+\left(\mathfrak{F}_{22} \mathfrak{y}^{2}, \mathfrak{y}^{2}\right)\right] .
$$

As before it is convenient to transform the hilbert space associated with the energy quadratic fork into the hilbert space $\mathfrak{F}$ with norm

$$
\left\|\mathfrak{y}^{\prime}\right\|=\left[\left\langle\mathfrak{y}^{\prime}, \mathfrak{y}^{\prime}\right\rangle\right]^{1 / 2}=\left[\left(\mathfrak{y}^{\prime 1}, \mathfrak{y}^{\prime 1}\right)+\int_{a}^{b}\left(\mathfrak{y}^{\prime 0}, \mathfrak{y}^{\prime 0}\right) d x+\left(\mathfrak{y}^{\prime 2}, \mathfrak{y}^{\prime 2}\right)\right]^{1 / 2}
$$

this is accomplished by the unitary mapping

$$
\mathfrak{y}^{i} \rightarrow \mathfrak{y}^{\prime i}=\mathfrak{F}_{i i}^{1 / 2} \mathfrak{y}^{i},
$$$$
i=0,1,2 \text {. }
$$

In terms of the transformed vectors, the equations (7.1) take the form

$$
\begin{aligned}
& \mathfrak{y}_{t}^{\prime 1}=\mathfrak{B}_{11}^{\prime} \mathfrak{q}^{\prime 1}+\mathfrak{B}_{10}^{\prime} \mathfrak{\eta}^{\prime 0}(a), \\
& \mathfrak{y}_{t}^{\prime 2}=\mathfrak{B}_{22}^{\prime} \mathfrak{\mathfrak { y }}^{\prime 2}+\mathfrak{B}_{20}^{\prime} \mathfrak{\eta}^{\prime 0}(b),
\end{aligned}
$$

${ }^{(10)}$ Suppose the equations of motion for the uncoupled mechanical system at the $i$ end are in canonical form, that is, $\dot{q}_{i}=\partial H / \partial p_{i}, \dot{p}_{i}=-\partial H / \partial q_{i}-\partial D / \partial \dot{q}_{i}$, where $H=\left(\sum_{i, j} t_{i j} p_{i} p_{j}\right) / 2$ $+\left(\sum_{i, j} v_{i j} q_{i} q_{j}\right) / 2$ and the dissipative function $D=\left(\sum_{i, j} d_{i j} \dot{q}_{i} \dot{q}_{i}\right) / 2$; the matrices $T=\left(t_{i j}\right)$ and $V=\left(v_{i j}\right)$ are positive definite, $D=\left(d_{i j}\right)$ is Hermitian, and $\dot{q}_{i}=\sum_{j} t_{i j} p_{i}$. To write this in the form (7.1) we set $E_{i i}=\left(\begin{array}{cc}V & 0 \\ 0 & T\end{array}\right) B_{i i}=\left(\begin{array}{cc}0 & V T \\ -T V & -T D T\end{array}\right)$. The condition $B_{i i}+B_{i i}^{*}=\left(\begin{array}{cc}0 & 0 \\ 0 & -2 T D T\end{array}\right) \leqq \Theta$ requires merely that $D \geqq \Theta$. 
where $\mathfrak{B}_{i t}^{\prime}=\mathfrak{F}_{i i}^{-1 / 2} \mathfrak{B}_{i i} \mathfrak{E}_{i i}^{-1 / 2}$ and $\mathfrak{B}_{i 0}^{\prime}=\mathfrak{F}_{i i}^{-1 / 2} \mathfrak{B}_{i 0}$ (the quotient spaces $\mathfrak{Y}_{a}$ and $\mathfrak{Y}_{b}$ can be thought of as invariant under this mapping). The condition (7.2) becomes

$$
\mathfrak{B}_{i i}^{\prime}+\mathfrak{B}_{i i}^{\prime *}=\mathfrak{E}_{i i}^{-1 / 2}\left(\mathfrak{B}_{i i}+\mathfrak{B}_{i i}^{*}\right) \mathfrak{E}_{i i}^{-1 / 2} \leqq \Theta, \quad i=1,2,
$$

equality holding in (7.5) if and only if it holds in (7.2). The effect of the mapping on the central system has already been described in $\$ 2$. Hereafter we work in the space $\mathfrak{S}$ and omit the primes.

The effect of the coupling on the end systems is given in (7.4). We have indicated that $\mathfrak{y}^{0}(a) \in \mathfrak{Y}_{a}$ and $\mathfrak{\eta}^{0}(b) \in \mathfrak{Y}_{b}$, but we have not said explicitly how $\mathfrak{y}^{0}(a)$ and $\mathfrak{y}^{0}(b)$ are to be obtained from $\mathfrak{y}$. Nor have we indicated the nature of the back-coupling effect of the end systems on the central system. Our choice of coupling mechanism will be based on the following heuristic considerations. We image the central system as extending past the points $a$ and $b$ and determine the coupling matrix $\mathfrak{B}_{20}$, say, so that the energy flow into the extended part of the central system at $b$ less the energy flow from the interior (i.e. $a<x<b)$ at $b$ is just equal to the energy cross-product term, namely $\left.\left(\mathfrak{B}_{20} \mathfrak{y}^{0}(b), \mathfrak{y}^{2}\right)+\left(\mathfrak{y}^{2}, \mathfrak{B}_{20} \mathfrak{y}\right)^{0}(b)\right)$. The effect of the mechanism at $b$ is to introduce a discontinuity in $\mathfrak{y}^{0}(x)$ at $b$ and we will take as our value of $\mathfrak{y}^{0}(b)$ in (7.4) the mean of the limits $\mathfrak{y}^{0}(b-)$ and $\mathfrak{y}^{0}(b+)$. The jump in $\mathfrak{y}^{0}(x)$ will of course vary linearly with $\mathfrak{y}^{2}$. Thus

$$
\left(\mathfrak{B}_{20} \mathfrak{y}^{0}(b), \mathfrak{y}^{2}\right)+\left(\mathfrak{y}^{2}, \mathfrak{B}_{20} \mathfrak{y}^{0}(b)\right)=\left(\mathfrak{A}_{00} \mathfrak{y}^{0}, \mathfrak{y}^{0}\right)^{b+}-\left(\mathfrak{A}_{00} \mathfrak{y}^{0}, \mathfrak{y}^{0}\right)^{b-},
$$

and

$$
\mathfrak{y}^{0}(b)=\frac{1}{2}\left[\mathfrak{y}^{0}(b+)+\mathfrak{y}^{0}(b-)\right], \quad \mathfrak{E}_{02} \mathfrak{\eta}^{2}=\mathfrak{y}^{0}(b+)-\mathfrak{y}^{0}(b-) .
$$

It is readily seen that this implies $\mathfrak{B}_{20}=\mathfrak{C}_{02} * \mathfrak{A}_{00}(b)$.

So far we have proceeded formally, our use of the quantities $\mathfrak{y}^{0}(b), \mathfrak{A}_{00}(b)$, etc., being certainly open to question. However, if we make use of the quotient space $\mathfrak{Y}_{b}$, it is actually a simple matter to assign a precise meaning to the above considerations. We set $\mathfrak{y}^{0}(b-)$ equal to the image of $\mathfrak{y}^{0}$ under the natural map of $\mathfrak{D}\left(L_{1}\right)$ onto $\mathfrak{Y}_{b}$. For any given linear transformation $\mathfrak{S}_{02}$ on $\mathfrak{Y}_{2}$ to $\mathfrak{Y}_{b}$, the relations $(7.7)$ uniquely determine $\mathfrak{y}^{0}(b)$ and $\mathfrak{y}^{0}(b+)$ (elements of $\mathfrak{Y}_{b}$ ) in terms of $\mathfrak{y}^{0}(b-)$ and $\mathfrak{y}^{2}$. In dealing with the adjoint operator, the element $z^{0}(b-)$ will likewise be defined as the image of $z^{0} \in \mathfrak{D}\left(M_{1}\right)$ in $Z_{b}$, and given a coupling transformation $\mathfrak{S}_{02}^{0}$ on $Z_{2}$ to $\bigcap_{b}$, the elements $z^{0}(b)$ and $z^{0}(b+)$ of $3_{b}$ will be defined by the analogues of (7.7). The coupling operators $\mathfrak{B}_{20}$ and $\mathfrak{B}_{20}^{0}$ on $\mathfrak{Y}_{b}$ to $\mathfrak{Y}_{2}$ and on $\mathfrak{B}_{b}$ to $\mathfrak{B}_{2}$, respectively, are defined by the relations

$$
\left(\mathfrak{y}^{2}, \mathfrak{B}_{20} \mathfrak{y}^{0}(b)\right)=\left(\mathfrak{P}_{00} \mathfrak{C}_{02} \mathfrak{y}^{2}, \mathfrak{y}^{0}\right)^{b} \text { and }\left(\mathfrak{z}^{2}, \mathfrak{B}_{203}^{0}{ }^{0}(b)\right)=\left(\mathfrak{H}_{00}\left(\mathfrak{C}_{02 \mathfrak{z}^{0}}^{0}, \mathfrak{z}^{0}\right)^{\mathfrak{b}}\right. \text {. }
$$

$\mathfrak{夭}_{02}$ and $\mathfrak{夭}_{02}^{0}$ become adjoint coupling transformations if 
(7.9) $\left(\mathfrak{y}^{2}, \mathfrak{B}_{20 \mathfrak{z}^{0}}^{0}(b)\right)=\left(\mathfrak{A}_{00}\left(\mathfrak{S}_{02} \mathfrak{y}^{2}, \mathfrak{z}^{0}\right)^{b}\right.$ and $\left(\mathfrak{z}^{2}, \mathfrak{B}_{20} \mathfrak{y}^{0}(b)\right)=\left(\mathfrak{A}_{00} \mathfrak{S}_{02 \mathfrak{z}^{0}}{ }^{2}, \mathfrak{y}^{0}\right)^{b}$.

It is, of course, required to show that the two relations in (7.9) are consistent. To this end, suppose $\left\{U_{b, i}\right\}$ and $\left\{V_{b, i}\right\}$ are a canonical pair of bases for $\mathfrak{V}_{b}$ and $\mathfrak{Z}_{b}$, respectively; and let $\mathfrak{\Xi}_{02} \mathfrak{y}_{i}^{2}=\sum c_{i \beta} U_{b, \beta}$ and $\mathfrak{夭}_{02}^{0} z_{i}^{2}=\sum c_{i \beta}^{0} V_{b, \beta}$, where $\mathfrak{y}_{i}^{2}=z_{i}^{2}$ are both $k_{2}$-tuples with $i$ th coordinate one and all other coordinates zero. Then the first relation in (7.9) is equivalent with $c_{i j}=\sum c_{i \beta}^{0}\left(\mathfrak{A}_{00} V_{b, \beta}, V_{b, j}\right)$ and the second is equivalent with $c_{i j}^{0}=\sum c_{i \beta}\left(\mathfrak{P}_{00} U_{b, \beta}, U_{b, j}\right)$; and the consistency of these two equations is assured by Theorem 5.4. The two relations in (7.9) combine to give $\mathfrak{B}_{20}^{0} \mathfrak{G}_{02}^{0}=\left(\mathfrak{B}_{20} \mathfrak{S}_{02}\right)^{*}$. Similar considerations naturally apply at the $a$ end.

We now write down a complete description of the augmented operator $\mathfrak{R}_{1}$ and its formal adjoint $\mathfrak{M}_{1}$, both of which are taken to be normalized so that the $\mathfrak{F}_{i i}=I$.

$$
\begin{aligned}
& {\left[\mathfrak{R}_{1} \mathfrak{y}\right]^{0}=\left(\mathfrak{A}_{00} \mathfrak{y}^{0}\right)_{x}+\mathfrak{B}_{00} \mathfrak{y}^{0},} \\
& {\left[\mathfrak{R}_{1} \mathfrak{y}\right]^{1}=\mathfrak{B}_{11} \mathfrak{y}^{1}+\mathfrak{B}_{10} \mathfrak{y}^{0}(a),} \\
& \left(\mathfrak{R}_{1} \mathfrak{y}\right]^{2}=\mathfrak{B}_{22} \mathfrak{y}^{2}+\mathfrak{B}_{20} \mathfrak{y}^{0}(b)
\end{aligned}
$$

where

$$
\begin{array}{ll}
\mathfrak{y}^{0}(a)=\frac{1}{2}\left[\mathfrak{y}^{0}(a+)+\mathfrak{y}^{0}(a-)\right], & \mathfrak{S}_{01} \mathfrak{h}^{1}=\mathfrak{y}^{0}(a+)-\mathfrak{y}^{0}(a-), \\
\mathfrak{y}^{0}(b)=\frac{1}{2}\left[\mathfrak{y}^{0}(b+)+\mathfrak{y}^{0}(b-)\right], & \left(\mathfrak{S}_{02} \mathfrak{y}^{2}=\mathfrak{y}^{0}(b+)-\mathfrak{y}^{0}(b-),\right. \\
\left(\mathfrak{y}^{1}, \mathfrak{B}_{10} \mathfrak{h}^{0}(a)\right)=\left(\mathfrak{A}_{00}\left(\mathfrak{S}_{01} \mathfrak{h}^{1}, \mathfrak{y}^{0}\right)^{a},\right. & \left(\mathfrak{y}^{2}, \mathfrak{B}_{20} \mathfrak{h}^{0}(b)\right)=\left(\mathfrak{A}_{00}\left(\mathfrak{S}_{02} \mathfrak{y}^{2}, \mathfrak{y}^{0}\right)^{b},\right. \\
\mathfrak{I}\left(\mathfrak{l}_{1}\right)=\left[\mathfrak{y} ; \mathfrak{y}^{0} \in \mathfrak{D}\left(L_{1}\right)\right] ; &
\end{array}
$$

and

$$
\begin{aligned}
& {\left[\mathfrak{M}_{1 \mathfrak{z}}\right]^{0}=-\left(\mathfrak{A}_{00 z^{0}}\right)_{x}+\left(\mathfrak{B}_{00}^{*}+\left(\mathfrak{A}_{00}\right)_{x}\right) \mathfrak{z}^{0},} \\
& {\left[\mathfrak{M}_{1 \mathfrak{z}}\right]^{1}=\mathfrak{B}_{11 z_{1}}^{*}-\mathfrak{B}_{10 \mathfrak{z}}^{0}(a) \text {, }} \\
& {\left[\mathfrak{M}_{1\}}\right]^{2}=\mathfrak{B}_{22 \xi_{2}}^{*}-\mathfrak{B}_{20\}}^{0}{ }^{0}(b) \text {, }}
\end{aligned}
$$

where

$$
\begin{aligned}
& z^{0}(a)=\frac{1}{2}\left[z^{0}(a+)+z^{0}(a-)\right], \quad \mathfrak{c}_{01 z^{0}}^{1}=z^{0}(a+)-z^{0}(a-), \\
& z^{0}(b)=\frac{1}{2}\left[z^{0}(b+)+z^{0}(b-)\right], \quad \mathfrak{S}_{02 z^{2}}^{2}=z^{0}(b+)-z^{0}(b-), \\
& \begin{aligned}
\left(\mathfrak{z}^{1}, \mathfrak{B}_{10 z}^{0}(a)\right) & =\left(\mathfrak{A}_{00}\left(\mathfrak{S}_{01 \mathfrak{z}}^{0}, \mathfrak{z}^{0}\right)^{a}, \quad\left(\mathfrak{z}^{2}, \mathfrak{B}_{20 z}^{0}{ }^{0}(b)\right)=\left(\mathfrak{A}_{00}\left(\mathfrak{S}_{02}{ }^{0}, \mathfrak{z}^{0}\right)^{b},\right.\right. \\
\mathfrak{I}\left(\mathfrak{M}_{1}\right) & =\left[\mathfrak{z} ; \mathfrak{z}^{0} \in \mathfrak{D}\left(M_{1}\right)\right]
\end{aligned}
\end{aligned}
$$


here $\mathfrak{S}_{01}, \mathfrak{S}_{01}^{0}$ and $\mathfrak{S}_{02}, \mathfrak{S}_{02}^{0}$ are each assumed to be pairs of adjoint coupling transformations. If we now set

$$
\mathfrak{B}=\left(\begin{array}{ccc}
\mathfrak{B}_{11} & 0 & 0 \\
0 & \mathfrak{B}_{00} & 0 \\
0 & 0 & \mathfrak{B}_{22}
\end{array}\right) \quad \text { and } \quad \mathfrak{A}=\left(\begin{array}{ccc}
0 & 0 & 0 \\
0 & \mathfrak{A}_{00} & 0 \\
0 & 0 & 0
\end{array}\right)
$$

then a straightforward calculation shows that

$$
\begin{aligned}
& \left\langle\mathfrak{l}_{1} \mathfrak{y}_{1}, \mathfrak{y}_{2}\right\rangle+\left\langle\mathfrak{y}_{1}, \mathfrak{R}_{1} \mathfrak{y}_{2}\right\rangle=\left\langle\left(\mathfrak{B}+\mathfrak{B}^{*}+\mathfrak{A}_{x}\right) \mathfrak{y}_{1}, \mathfrak{y}_{2}\right\rangle-\left(\mathfrak{A}_{00} \mathfrak{y}_{1}^{0}, \mathfrak{\mathfrak { y }}_{2}\right)^{a-} \\
& +\left(\mathfrak{A}_{00} \mathfrak{\eta}_{1}^{0}, \mathfrak{y}_{2}^{0}\right)^{b+} \text {, } \\
& \left\langle\mathfrak{R}_{1} \mathfrak{y}, \mathfrak{z}\right\rangle-\left\langle\mathfrak{y}, \mathfrak{M}_{1 \mathfrak{z}}\right\rangle=-\left(\mathfrak{A}_{00} \mathfrak{y}^{0}, z^{0}\right)^{a-}+\left(\mathfrak{A}_{00} \mathfrak{y}^{0}, \mathfrak{z}^{0}\right)^{b+}, \\
& \mathfrak{y}_{1}, \mathfrak{y}_{2} \in \mathfrak{D}\left(\mathfrak{R}_{1}\right) ; \\
& \mathfrak{y} \in \mathfrak{D}\left(\mathfrak{R}_{1}\right), \mathfrak{z} \in \mathfrak{D}\left(\mathfrak{M}_{1}\right) ; \\
& \left\langle\mathfrak{M}_{1 z_{1}, z_{2}}\right\rangle+\left\langle\mathfrak{z}_{1}, \mathfrak{M}_{1 z_{2}}\right\rangle=\left\langle\left(\mathfrak{B}+\mathfrak{B}^{*}+\mathfrak{A}_{x}\right)_{z_{1}, z_{2}}\right\rangle+\left(\mathfrak{A}_{00 z_{1}, z_{2}}^{0}\right)^{0} \\
& -\left(\mathfrak{A}_{00 z_{1}}^{0}, \mathfrak{z}_{2}^{0}\right){ }^{b+} \text {, } \\
& z_{1}, z_{2} \in \mathfrak{D}\left(\mathfrak{M}_{1}\right) \text {. }
\end{aligned}
$$

In view of these relations, the obvious dissipative condition is

$$
\mathfrak{B}+\mathfrak{B}^{*}+\mathfrak{A}_{x} \leqq \Theta,
$$

which hereafter will be assumed. For the same reason, we say that a function $\mathfrak{y} \in \mathfrak{D}\left(\Omega_{1}\right)$ satisfies a dissipative boundary condition if

$$
-\left(\mathfrak{A}_{00} \mathfrak{y}^{0}, \mathfrak{y}^{0}\right)^{a-}+\left(\mathfrak{A}_{00} \mathfrak{y}^{0}, \mathfrak{y}^{0}\right)^{b+} \leqq 0,
$$

and, similarly, a function $z \in \mathfrak{D}\left(\mathfrak{M}_{1}\right)$ satisfies dissipative boundary conditions if

$$
\left(\mathfrak{H}_{00 z^{0}}, z^{0}\right)^{a-}-\left(\mathfrak{A}_{00 z^{0}}, z^{0}\right)^{b+} \leqq 0 .
$$

With these preliminaries out of the way, we now proceed to find all dissipative restrictions of $\mathfrak{R}_{1}$ and $\mathfrak{M}_{1}$ which generate strongly continuous semigroups of operators. The argument follows the same pattern as before and we begin by investigating the solutions of the homogeneous systems of equations

$$
\begin{aligned}
& \text { (a) } \lambda \mathfrak{y}^{0}-\left(\mathfrak{A}_{00} \mathfrak{y}^{0}\right)_{x}-\mathfrak{B}_{00} \mathfrak{y}^{0}=\theta, \\
& \text { (b) } \lambda \mathfrak{y}^{1}-\mathfrak{B}_{11} \mathfrak{y}^{1}-\mathfrak{B}_{10} \mathfrak{y}^{0}(a)=\theta, \\
& \text { (c) } \lambda \mathfrak{y}^{2}-\mathfrak{B}_{22} \mathfrak{y}^{2}-\mathfrak{B}_{20} \mathfrak{y}^{0}(b)=\theta,
\end{aligned}
$$

and

$$
\begin{aligned}
& \text { (a) } \lambda_{z}{ }^{0}+\left(\mathfrak{A}_{00 z^{0}}\right)_{x}-\left(\mathfrak{B}_{00}^{*}+\left(\mathfrak{P}_{00}\right)_{x}\right)_{z}{ }^{0}=\theta, \\
& \text { (b) } \lambda_{z}{ }^{1}-\mathfrak{B}_{11 z}^{*}+\mathfrak{B}_{10 z}^{0}{ }^{0}(a)=\theta, \\
& \text { (c) } \lambda_{z}{ }^{2}-\mathfrak{B}_{22 z}^{*}{ }^{2}+\mathfrak{B}_{20 z}^{0}{ }^{0}(b)=\theta .
\end{aligned}
$$


By the convention mentioned at the close of $\$ 5$ a meaning can be assigned to $\mathfrak{B}_{10} \mathfrak{y}^{0}(a)$ if $\mathfrak{y}^{0} \in F_{a}$ and to $\mathfrak{B}_{20} \mathfrak{y}^{0}(b)$ if $\mathfrak{y}^{0} \in F_{b}$; however if $\mathfrak{y}^{0} \in F_{b}$ but not to $F_{a}$ then $(7.18 \mathrm{~b})$ will be meaningless and we will be content with simply the $\left(\mathfrak{y}^{0}, \mathfrak{y}^{2}\right)$ components of the solution. Similar remarks apply at the $a$ end and for the system (7.19).

To pursue this matter a bit further, let $\mathfrak{y}^{0}$ be a solution of (7.18a) which lies in $F_{b}$ and hence maps into $\mathfrak{y}^{0}(b-) \in \mathfrak{Y}_{b}$. We now choose $\mathfrak{y}^{2}$ so that $(7.18 \mathrm{c})$ is satisfied for $\mathfrak{y}^{0}(b)$ defined as in (7.7). In fact, substituting for $\mathfrak{y}^{0}(b)$ in terms of $\mathfrak{y}^{0}(b-)$ and $\mathfrak{y}^{2}$, we obtain

$$
\lambda \mathfrak{y}^{2}-\mathfrak{B}_{22} \mathfrak{h}^{2}-\mathfrak{B}_{20} \mathfrak{\complement}_{02} \mathfrak{\eta}^{2} / 2=\mathfrak{B}_{20} \mathfrak{\eta}^{0}(b-) .
$$

If $\lambda$ is not a characteristic value of the matrix

$$
\mathfrak{B}_{22}+\mathfrak{B}_{20} \mathfrak{C}_{02} / 2,
$$

then (7.20) will have a unique solution $\mathfrak{y}^{2}$ for each $\mathfrak{\eta}^{0}(b-)$ and, in particular, for $\mathfrak{y}^{0}(b-)=\theta$ we have $\mathfrak{y}^{2}=\theta$ and $\mathfrak{y}^{0}(b+)=\mathfrak{y}^{0}(b-)+\left(\mathfrak{C}_{02} \mathfrak{\eta}^{2}=\theta\right.$. Moreover for the so obtained solution $\mathfrak{y}_{b}=\left(-, \mathfrak{y}^{0}, \mathfrak{y}^{2}\right)$ of $(7.18 \mathrm{a})$ and $(7.18 \mathrm{c})$, we will have

$$
2 \lambda\left(\mathfrak{y}^{2}, \mathfrak{y}^{2}\right)=\left(\left(\mathfrak{B}_{22}^{*}+\mathfrak{B}_{22}\right) \mathfrak{y}^{2}, \mathfrak{y}^{2}\right)+\left(\mathfrak{A}_{00} \mathfrak{y}^{0}, \mathfrak{y}^{0}\right)^{b+}-\left(\mathfrak{A}_{00} \mathfrak{y}^{0}, \mathfrak{y}^{0}\right)^{b-},
$$

and as a consequence

$$
2 \lambda\left(\mathfrak{y}^{2}, \mathfrak{y}^{2}\right) \leqq\left(\mathfrak{A}_{00} \mathfrak{y}^{0}, \mathfrak{y}^{0}\right)^{b+}-\left(\mathfrak{A}_{00 \mathfrak{y}^{0}}, \mathfrak{y}^{0}\right)^{b-} .
$$

On the other hand, by substituting in (7.18c) for $\mathfrak{y}^{0}(b)$ in terms of $\mathfrak{y}^{0}(b+)$ and $\mathfrak{y}^{2}$, we see that $\mathfrak{y}^{2}$ also satisfies

$$
\lambda \mathfrak{y}^{2}-\mathfrak{B}_{22} \mathfrak{\eta}^{2}+\mathfrak{B}_{20} \mathfrak{S}_{02} \mathfrak{\eta}^{2} / 2=\mathfrak{B}_{20} \mathfrak{\eta}^{0}(b+) .
$$

Thus if $\lambda$ is not a characteristic value of

$$
\mathfrak{B}_{22}-\mathfrak{B}_{20} \mathfrak{C}_{02} / 2
$$

then $\mathfrak{y}^{0}(b+)=\theta$ implies that $\mathfrak{y}^{2}=\theta$ and hence that $\mathfrak{y}^{0}(b-)=\mathfrak{y}^{0}(b+)-\mathfrak{C}_{02} \mathfrak{y}^{2}=\theta$. Thus, dimensionwise, the linear space spanned by the $\mathfrak{n}^{0}(b+)$ is at least as large as that spanned by the $\mathfrak{y}^{0}(b-)$. Now according to the corollary to Theorem 5.3 , as $\mathfrak{y}^{0}$ ranges over $F_{b}$, the corresponding $\mathfrak{y}^{0}(b-)$ span $\mathfrak{V}_{b}$, and it follows from the above that the same is true of the $\mathfrak{y}^{0}(b+)$.

A similar situation prevails at the $a$ end for $\mathfrak{y}^{0} \in F_{a}$. In this case a unique solution $\left.\mathfrak{y}_{a}=\left(\mathfrak{y}^{1}, \mathfrak{y}\right)^{0},-\right)$ exists for $(7.18 \mathrm{a})$ and $(7.18 \mathrm{~b})$ with

$$
2 \lambda\left(\mathfrak{y}^{1}, \mathfrak{y}^{1}\right)=\left(\left(\mathfrak{B}_{11}+\mathfrak{B}_{11}^{*}\right) \mathfrak{y}^{1}, \mathfrak{y}^{1}\right)+\left(\mathfrak{A}_{00} \mathfrak{y}^{0}, \mathfrak{y}^{0}\right)^{a_{+}}-\left(\mathfrak{A}_{00} \mathfrak{y}^{0}, \mathfrak{y}^{0}\right)^{a_{-}}
$$

providing $\lambda$ is not a characteristic value of the matrix

$$
\mathfrak{B}_{11}-\mathfrak{B}_{10} \mathfrak{E}_{01} / 2 ;
$$

and again the $\mathfrak{y}^{0}(a-)$ span $\mathfrak{Y}_{a}$ as $\mathfrak{y}^{0}$ ranges over $F_{a}$ when $\lambda$ is not a characteristic value of 


$$
\mathfrak{B}_{11}+\mathfrak{B}_{10} \mathfrak{E}_{01} / 2 \text {. }
$$

Applying the same reasoning to the adjoint homogeneous system, we obtain for $z^{0} \in G_{b}$ [or $G_{a}$ ] a unique solution $z_{b}=\left(-, z^{0}, z^{2}\right)$ [or $\left.z_{a}=\left(z^{1}, z^{0},-\right)\right]$ with

$$
2 \lambda\left(\mathfrak{z}^{2}, z^{2}\right)=\left(\left(\mathfrak{B}_{22}+\mathfrak{B}_{22}^{*}\right) z^{2}, z^{2}\right)-\left(\mathfrak{A}_{00 z^{0}}, z^{0}\right)^{b+}+\left(\mathfrak{A}_{00 z^{0}}, z^{0}\right)^{b-}
$$

[or

$$
\text { (7.25a) } \left.2 \lambda\left(\mathfrak{z}^{1}, z^{1}\right)=\left(\left(\mathfrak{B}_{11}+\mathfrak{B}_{11}^{*}\right) z^{1}, z^{1}\right)-\left(\mathfrak{A}_{00 z^{0}}, z^{0}\right)^{a+}+\left(\mathfrak{A}_{00 z^{0}}, z^{0}\right)^{a-}\right],
$$

providing $\lambda$ is not a characteristic value of

$$
\mathfrak{B}_{22}^{*}-\mathfrak{B}_{20}^{0} \mathfrak{S}_{02}^{0} / 2\left[\text { or } \mathfrak{B}_{11}^{*}+\mathfrak{B}_{10}^{0} \mathfrak{G}_{01}^{0} / 2\right] .
$$

Likewise the $z^{0}(b+)\left[z^{0}(a-)\right]$ span $\not_{b}$ [or $\left.Z_{a}\right]$ as $z^{0}$ ranges over $G_{b}\left[G_{a}\right]$ when $\lambda$ is not a characteristic value of

$$
\mathfrak{B}_{22}^{*}+\mathfrak{B}_{20}^{0} \mathfrak{B}_{02}^{0} / 2\left[\text { or } \mathfrak{B}_{11}^{*}-\mathfrak{B}_{10}^{0}\left(\mathfrak{S}_{01}^{0} / 2\right] .\right.
$$

We note that the matrices (7.26) and (7.27) are the adjoints of (7.24) and (7.21), respectively.

Finally suppose that $\mathfrak{y}_{b}=\left(-, \mathfrak{y}^{0}, \mathfrak{y}^{2}\right)$ and $z_{b}=\left(-, z^{0}, z^{2}\right)$ are solutions in the above sense of $(7.18 \mathrm{a}, \mathrm{c})$ and $(7.19 \mathrm{a}, \mathrm{c})$ respectively. According to Theorem 3.2, $\left(\mathfrak{A}_{00} \mathfrak{\eta}^{0}, z^{0}\right)^{x}$ is constant on $(a, b)$. Moreover, making use of $(7.18 \mathrm{c})$ and (7.19c) we obtain $\left(\mathfrak{h}^{2}, \mathfrak{B}_{20 z^{0}}^{0}(b)\right)+\left(\mathfrak{B}_{20} \mathfrak{H}^{0}(b), z^{2}\right)=0$, which together with (7.9) gives $\left(\mathfrak{A}_{00} \mathfrak{G}_{02} \mathfrak{h}^{2}, z^{0}\right)^{b}+\left(\mathfrak{h}^{0}, \mathfrak{A}_{00} \mathfrak{G}_{02 z^{0}}^{0}\right)^{b}=0$. Applying (7.11) and (7.13) we see that

$$
\left(\mathfrak{A}_{00} \mathfrak{\eta}^{0}, z^{0}\right)^{b+}=\left(\mathfrak{A}_{00} \mathfrak{\eta}^{0}, z^{0}\right)^{b-}=\left(\mathfrak{A}_{00} \mathfrak{y}^{0}, z^{0}\right)^{x} \quad \text { for all } x \in(a, b) .
$$

Likewise for $\mathfrak{y}_{a}=\left(\mathfrak{y}^{1}, \mathfrak{y}^{0},-\right)$ and $z_{a}=\left(z^{1}, z^{0},-\right)$ solutions of $(7.18 \mathrm{a}, b)$ and $(7.19 \mathrm{a}, \mathrm{b})$ respectively, we have

$$
\left(\mathfrak{A}_{00} \mathfrak{y}^{0}, z^{0}\right)^{a_{-}}=\left(\mathfrak{A}_{00} \mathfrak{y}^{0}, z^{0}\right)^{a_{+}}=\left(\mathfrak{A}_{00} \mathfrak{y}^{0}, z^{0}\right)^{x}
$$

for all $x \in(a, b)$.

We note that $\eta^{0} \in F_{a} \cap F_{b}$ can be continued at both ends to define a solution $\mathfrak{y}=\left(\mathfrak{y}^{1}, \mathfrak{y}^{0}, \mathfrak{y}^{2}\right)$ of $(7.18 \mathrm{a}, \mathrm{b}, \mathrm{c})$, and the analogous assertion holds for $z^{0} \in G_{a} \cap G_{b}$. In this connection we have the following lemma which is required for $\S 8$.

Lemma 7.1. Suppose $\lambda$ is not a characteristic value of $(7.21 \mathrm{a}, \mathrm{b})$ and let $\mathrm{y}_{a}$ and $\mathfrak{y}_{b}$ be solutions in the above sense of $(7.18 \mathrm{a}, \mathrm{b})$ and $(7.18 \mathrm{a}, \mathrm{c})$, respectively. If $\mathfrak{y}_{a}^{0}(x)=\mathfrak{y}_{b}^{0}(x)$ for some $x \in(a, b)$ and if $-\left(\mathfrak{H}_{00} \mathfrak{y}_{a}^{0}, \mathfrak{y}_{a}^{0}\right)^{a-}+\left(\mathfrak{H}_{00} \mathfrak{y}_{b}^{0}, \mathfrak{y}_{b}^{0}\right)^{b+} \leqq 0$, then $\mathfrak{h}_{a} \equiv \theta \equiv \mathfrak{h}_{b}$.

Proof. Since $\mathfrak{y}_{a}^{0}$ and $\mathfrak{y}_{b}^{0}$ are both solutions of $(7.18 \mathrm{a})$ it follows by the uniqueness theorem that $\mathfrak{y}_{a}^{0}(x)=\mathfrak{y}_{b}^{0}(x)$ for all $x \in(a, b)$. Thus $\mathfrak{u}^{0} \equiv \mathfrak{\eta}_{a}^{0}=\mathfrak{y}_{b}^{0}$, $\mathfrak{u}^{1} \equiv \mathfrak{y}_{a}^{\mathfrak{l}}, \mathfrak{u}^{2} \equiv \mathfrak{y}_{b}^{2}$ is a solution of $(7.18 \mathrm{a}, \mathrm{b}, \mathrm{c})$ and $\mathfrak{u}=\left(\mathfrak{u}^{1}, \mathfrak{u}^{0}, \mathfrak{u}^{2}\right)$ clearly belongs 
to $\mathfrak{T}\left(\mathfrak{Q}_{1}\right)$. Obviously $\mathfrak{u}^{0}(a-)=\mathfrak{y}_{a}^{0}(a-)$ and $\mathfrak{u}^{0}(b+)=\mathfrak{y}_{b}^{0}(b+)$ so that

$$
-\left(\mathfrak{A}_{00} \mathfrak{u}^{0}, \mathfrak{u}^{0}\right)^{a-}+\left(\mathfrak{H}_{00} \mathfrak{u}^{0}, \mathfrak{u}^{0}\right)^{b+} \leqq 0 .
$$

Hence (7.14) implies that $\|\mathfrak{u}\|=0$, which proves the lemma.

In constructing the required restrictions of $\ell_{1}$ and $\mathfrak{M}_{1}$ we again make use of the product spaces $\mathfrak{Y}_{a, b}$ and $\mathfrak{Z}_{a, b}$, the operator $\mathfrak{H}_{a, b}$, and the $\mathfrak{A}_{a, b}$-orthogonal complements $\mathfrak{R}_{a, b}$ and $\mathfrak{P}_{a, b}$, defined at the beginning of $\S 6$. We now have

TheOREM 7.1. Suppose $\mathfrak{N}_{a, b}$ and $\mathfrak{P}_{a, b}$ are $\mathfrak{A}_{a, b}$-orthogonal complements satisfying the conditions (6.2) and (6.4). Let $\mathfrak{R}$ and $\mathfrak{M}$ be restrictions of $\mathfrak{R}_{1}$ and $\mathfrak{M}_{1}$, respectively, with domains

$$
\begin{aligned}
\mathfrak{D}(\mathfrak{l}) & =\left[\mathfrak{y} ; \mathfrak{y} \in \mathfrak{D}\left(\mathfrak{R}_{1}\right),\left[\mathfrak{y}^{0}(a-), \mathfrak{y}^{0}(b+)\right] \rightarrow \mathfrak{R}_{a, b}\right], \\
\mathfrak{D}(\mathfrak{M}) & =\left[\mathfrak{z} ; \mathfrak{z} \in \mathfrak{D}\left(\mathfrak{M}_{1}\right),\left[\mathfrak{z}^{0}(a-), \mathfrak{z}^{0}(b+)\right] \rightarrow \mathfrak{P}_{a, b}\right],
\end{aligned}
$$

7 hen $\mathfrak{R}$ and $\mathfrak{M}$ are dissipative operators satisfying the Hille-Yosida criterion for all $\lambda>0$. Moreover, $\mathfrak{R}=\mathfrak{M}^{*}$ and $\mathfrak{M}=\mathfrak{\Omega}^{*}$.

Proof. Suppose first that $\lambda>0$ is larger than any of the characteristic values of the matrices (7.21) and (7.24). We can then construct solution pairs $\left[\mathfrak{y}_{a}, \mathfrak{y}_{b}\right]$ and $\left[z_{a}, z_{b}\right]$ of the homogeneous systems (7.18) and (7.19) respectively, at least in the sense considered above. Let $N_{a, b}$ be the set of solution pairs $\left[\mathfrak{y}_{a}, \mathfrak{y}_{b}\right]$ which map into $\mathfrak{R}_{a, b}$, that is, for which $\left[\mathfrak{y}_{a}^{0}(a-), \mathfrak{y}_{n}^{0}(b+)\right] \in \mathfrak{R}_{a, b}$, and let $P_{a, b}$ be the set of all solution pairs $\left[z_{a}, z_{b}\right]$ which map into $\mathfrak{P}_{a, b}$. It is clear that $N_{a, b}$ actually maps onto $\mathfrak{N}_{a, b}$ and that $P_{a, b}$ maps onto $\mathfrak{P}_{a, b}$. In order to determine the dimensionality of $N_{a, b}$ and $P_{a, b}$ we proceed as in the proof of Theorem 6.1. Now $r-m_{a}$ linearly independent solutions of (7.18a) map into $\mathfrak{D}\left(L_{a}\right)$ and $r-m_{b}$ linearly independent solutions map into $\mathfrak{D}\left(L_{b}\right)$. Since $\mathfrak{y}^{0}(a+)=\theta$ if and only if $\mathfrak{y}^{0}(a-)=\theta$ and $\mathfrak{y}^{0}(b-)=\theta$ if and only if $\mathfrak{y}^{0}(b+)=\theta$, we see that for $\left(2 r-m_{a}-m_{b}\right)$ linearly independent solution pairs the corresponding $\left[\mathfrak{y}^{0}(a-), \mathfrak{y}^{0}(b+)\right]$ coincides with the zero element of $\mathfrak{Y}_{a, b}$. On the other hand the $\mathfrak{y}^{0}(a-)$ span $\mathfrak{y}_{a}$ as $\mathfrak{y}^{0}$ ranges over $F_{a}$ and the $\mathfrak{y}^{0}(b+)$ span $\mathfrak{V}_{b}$ as $\mathfrak{y}^{0}$ ranges over $F_{b}$. It follows that $N_{a, b}$ is of dimension

$$
\left(m_{a}+m_{b}-r\right)-\left(2 r-m_{a}-m_{b}\right)=r
$$

and, similarly, $P_{a, b}$ is also of dimension $r$. Further for any solution pair $\left[\mathfrak{y}_{a}, \mathfrak{y}_{b}\right] \in N_{a, b}$ the relations (6.2) and (7.22) imply that

$$
-\left(\mathfrak{A}_{00} \mathfrak{y}_{a}^{0}, \mathfrak{y}_{a}^{0}\right)^{a+}+\left(\mathfrak{A}_{00} \mathfrak{y}_{b}^{0}, \mathfrak{y}_{b}^{0}\right)^{b-} \leqq-\left(\mathfrak{P}_{00} \mathfrak{y}_{a}^{0}, \mathfrak{y}_{a}^{0}\right)^{a-}+\left(\mathfrak{A}_{00} \mathfrak{y}_{b}^{0}, \mathfrak{y}_{b}^{0}\right)^{b+} \leqq 0,
$$

whereas for any $\left[z_{a}, z_{b}\right] \in P_{a, b}(6.4)$ and (7.25) imply that

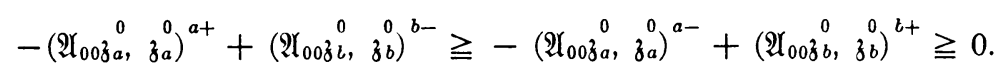


Finally we see from (7.28) that the sets $N_{a, b}$ and $P_{a, b}$ are $A_{a, b}$-orthogonal and, because of their dimension, actually $A_{a, b}$-orthogonal complements. Hence these sets are of the type employed in Theorem 4.1.

Before writing down a form of the green's function for the inhomogeneous system, we first choose a set of $r$ linearly independent solution pairs $\left[\mathfrak{y}_{a, i}, \mathfrak{y}_{b, i}\right]$ spanning $N_{a, b}$ in the manner discussed at the end of $\$ 4$ in connection with the representation of $R_{2}(\lambda)$ as given in (4.23). The $r$ linearly independent solution pairs $\left[z_{a, i}, z_{b, i}\right]$ spanning $P_{a, b}$ are then selected so as to satisfy (3.18). As we have seen, this will result in $z_{a, i}^{0}=\theta$ for $i \in s_{2}$ and $z_{b, i}^{0}=\theta$ for $i \in s_{3}$. Denoting the so defined $R_{2}(\lambda)$ by $\Re_{00}(\lambda)$, we now show that the resolvent for the operator $\mathfrak{R}$ can be represented as

$$
\begin{aligned}
& {[\Re(\lambda) \mathfrak{f}]^{0}=\Re_{00}(\lambda) f^{0}-\sum_{i \in s_{1}}\left(\mathfrak{y}_{a, i}^{0}+\mathfrak{y}_{b, i}^{0}\right)\left[\left(f^{1}, \frac{1}{z_{a, i}}\right)+\left(f^{2},{ }_{z b i}^{2}\right)\right],} \\
& {[\Re(\lambda) \mathfrak{f}]^{1}=\Re_{11}(\lambda) \mathfrak{f}^{1}-\sum_{i \in s_{1} \cup_{s_{2}}}\left(\mathfrak{h}_{a, i}^{1}+\mathfrak{y}_{b, i}^{1}\right) \int_{a}^{b}\left(\mathfrak{f}^{0},{ }_{b}^{0} b, i\right) d \xi} \\
& -\sum_{i \in s_{1}}\left(\mathfrak{h}_{a, i}^{1}+\mathfrak{y}_{b, i}^{1}\right)\left[\left(\mathfrak{f}^{1}, \underset{z a, i}{1}\right)+\left(\mathfrak{f}^{2}, \underset{z b, i}{2}\right)\right],
\end{aligned}
$$

$$
\begin{aligned}
{[\Re(\lambda) \mathfrak{f}]^{2}=} & \Re_{22}(\lambda) \mathfrak{f}^{2}-\sum_{i \in s_{1} \cup_{s_{3}}}\left(\mathfrak{y}_{a, i}^{2}+\mathfrak{y}_{b, i}^{2}\right) \int_{a}^{b}\left(\mathfrak{f}^{0}, z_{a, i}^{0}\right) d \xi \\
& -\sum_{i \in s_{1}}\left(\mathfrak{y}_{a, i}^{2}+\mathfrak{y}_{b, i}^{2}\right)\left[\left(\mathfrak{f}^{1}, z_{a, i}^{1}\right)+\left(\mathfrak{f}^{2}, \underset{z_{b, i}}{2}\right)\right],
\end{aligned}
$$

where

$$
\begin{aligned}
& \Re_{11}(\lambda)=\left[\lambda I-\mathfrak{B}_{11}+\mathfrak{B}_{10} \mathfrak{\complement}_{01} / 2\right]^{-1}, \\
& \mathfrak{R}_{22}(\lambda)=\left[\lambda I-\mathfrak{B}_{22}-\mathfrak{B}_{20} \mathfrak{\complement}_{02} / 2\right]^{-1} .
\end{aligned}
$$

In order to verify that $\Re(\lambda)$ really defines the resolvent $\Re(\lambda ; \mathfrak{R})$ we note first of all that for $\mathfrak{y} \in \mathfrak{D}\left(\mathfrak{R}_{1}\right),-\left(\mathfrak{A}_{00} \mathfrak{y}^{0}, \mathfrak{y}^{0}\right)^{a-}+\left(\mathfrak{A}_{00} \mathfrak{y}^{0}, \mathfrak{y}^{0}\right)^{b+} \leqq 0$, and $\mathfrak{f}=\lambda \mathfrak{y}-\mathfrak{R}_{1} \mathfrak{y}$, the first relation in (7.14) implies that

$$
\lambda\|\mathfrak{h}\| \leqq\|f\| .
$$

In particular this holds for all $\mathfrak{y \in D}(\mathfrak{R})$.

Suppose now that $\mathrm{f}^{1}=\theta=\mathfrak{f}^{2}$ and that $\mathfrak{f}^{0} \in L_{2}(a, b ; I)$ vanishes outside of $a<a^{\prime}<x<b^{\prime}<b$. Then all of the terms in (7.30) are well defined and it is clear that $\mathfrak{y} \equiv \Re(\lambda) \mathfrak{f}$ belongs to $\mathfrak{D}\left(\Omega_{1}\right)$. Moreover one sees directly from (4.23) that $\mathfrak{y}^{0}$ behaves like

$$
-\sum_{s_{1} \cup}\left(\mathfrak{y}_{a, i}^{0}+\mathfrak{y}_{b, i}^{0}\right) \int_{a}^{b}\left(f^{0}, z_{b, i}^{0}\right) d \xi
$$

for $x<a^{\prime}$ and like 


$$
-\sum_{s_{1}} \bigcup_{s_{3}}\left(\mathfrak{y}_{a, i}^{0}+\mathfrak{y}_{b, i}^{0}\right) \int_{a}^{b}\left(f^{0},{ }_{z b, i}^{0}\right) d \xi
$$

for $x>b^{\prime}$. The only nonvanishing terms in (7.30) for $y^{1}$ are those which together with (7.33) satisfy the homogeneous system $(7.18 \mathrm{a}, \mathrm{b})$, whereas the only nonvanishing terms for $\mathfrak{y}^{2}$ are those which together with (7.34) satisfy (7.18a, c). Thus $\Re(\lambda) \mathfrak{f}$ is a particular solution of $\lambda \mathfrak{y}-\mathfrak{R}_{1} \mathfrak{y}=\mathfrak{f}$. Furthermore, for $x_{1}<a^{\prime}$ and $x_{2}>b^{\prime}$ we have just as in the proof of Theorem 4.1

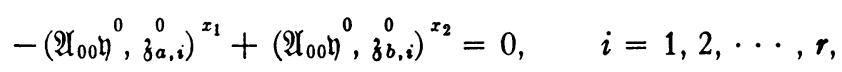

and because of (7.28) this continues to hold for $x_{1}$ replaced by $a-$ and $x_{2}$ replaced by $b+$. As a consequence $\left[\mathfrak{y}^{0}(a-), \mathfrak{y}^{0}(b+)\right]$ is $\mathfrak{A}_{a, b}$-orthogonal to $\mathfrak{P}_{a, b}$ and therefore lies in $\mathfrak{R}_{a, b}$. Thus $\mathfrak{y} \in \mathfrak{D}(\mathfrak{l})$ and hence (7.32) is satisfied.

More generally, suppose $f^{1}=\theta=f^{2}$ and that $f^{0}$ is an arbitrary element of $L_{2}(a, b ; I)$. Then we can approximate $f$ in $\mathfrak{S}$ by a sequence $\mathfrak{f}_{n}$ of the above type. Setting $\mathfrak{y}_{n}=\mathfrak{i}(\lambda) \mathfrak{f}_{n}$, we see that $\mathfrak{y}_{n} \in \mathfrak{D}(\mathfrak{l})$ and $\lambda\left\|\mathfrak{y}_{n}-\mathfrak{y}_{m}\right\| \leqq\left\|\mathfrak{f}_{n}-\mathfrak{f}_{m}\right\|$. In particular the sequence, $\left\{\eta_{n}^{0}\right\}$ converges to a limit, say $\mathfrak{y}^{0}$, in $H$ and, applying Lemma 5.2, we see that $\mathfrak{y}^{0} \in \mathfrak{D}\left(L_{1}\right)$ and $\lambda \mathfrak{y}^{0}-L_{1} \mathfrak{y}^{0}=\mathfrak{f}^{0}$. On the other hand it is clear from the representation $(4.23)$ that $\mathfrak{y}^{0}=[\Re(\lambda) \mathfrak{f}]^{0}$. In case $[\Re(\lambda) \mathfrak{f}]^{1}$ and $[\Re(\lambda) \mathfrak{f}]^{2}$ are well defined as given in (7.30), then $\mathfrak{y}^{1}=\lim _{n} \mathfrak{y}_{n}^{1}$ and $\mathfrak{y}^{2}$ $=\lim _{n} y_{n}^{0}$. In the contrary case the Banach-Steinhaus Theorem asserts that we can define $\mathfrak{y}^{1}$ and $\mathfrak{y}^{2}$ by these limits. According to Lemma 4.3 we have $\mathfrak{y}_{n}^{0}(a+) \rightarrow \mathfrak{y}^{0}(a+)$ and $\mathfrak{y}_{n}^{0}(b-) \rightarrow \mathfrak{y}^{0}(b-)$. It now follows from (7.11) that $\mathfrak{y}_{n}^{0}(a-) \rightarrow \mathfrak{y}^{0}(a-)$ and $\mathfrak{y}_{n}^{0}(b+) \rightarrow \mathfrak{y}^{0}(b+)$ and it is clear from this that $\mathfrak{y} \in \mathfrak{D}(\mathfrak{R})$.

It remains to consider the case $f^{0} \equiv \theta$ with arbitrary $f^{1}$ and $f^{2}$. In this case all of the terms in (7.30) are well defined and it is clear that $\mathfrak{y}=\Re(\lambda) \mathfrak{f} \in \mathfrak{D}\left(\Omega_{1}\right)$. We shall verify that $\mathfrak{y}$ is a particular solution of $\lambda \mathfrak{y}-\Omega_{1} \mathfrak{y}=f$ and that the boundary conditions are satisfied. Now $\mathfrak{y}$ differs from the function $\mathfrak{u}$ defined as

$$
\mathfrak{u}^{1}=\Re_{11}(\lambda) \mathfrak{f}^{1}, \quad \mathfrak{u}^{0} \equiv \theta \text { for } x \in(a, b), \quad \mathfrak{u}^{2}=\mathfrak{R}_{22}(\lambda) \mathfrak{f}^{2}
$$

by a solution of the homogeneous system which belongs to $\mathfrak{D}\left(\mathfrak{R}_{1}\right)$. On the other hand if we set $\mathfrak{u}^{0}(a-)=-\mathfrak{\complement}_{01} \mathfrak{u}^{1}$ and $\mathfrak{u}^{0}(b+)=\mathfrak{C}_{02} \mathfrak{u}^{2}$, we see that

$$
\lambda \mathfrak{u}^{1}-\mathfrak{B}_{11} \mathfrak{u}^{1}-\mathfrak{B}_{10} \mathfrak{H}^{0}(a)=\left[\lambda I-\mathfrak{B}_{11}+\mathfrak{B}_{10} \mathfrak{S}_{01} / 2\right] \mathfrak{u}^{1}=\mathfrak{f}^{1}
$$

and

$$
\lambda \mathfrak{u}^{2}-\mathfrak{B}_{22} \mathfrak{u}^{2}-\mathfrak{B}_{20} \mathfrak{u}^{0}(b)=\left[\lambda I-\mathfrak{B}_{22}-\mathfrak{B}_{20} \mathfrak{S}_{02} / 2\right] \mathfrak{u}^{2}=\mathfrak{f}^{2} .
$$

Consequently $\lambda \mathfrak{y}-\mathfrak{R}_{1} \mathfrak{y}=\lambda \mathfrak{u}-\mathfrak{R}_{1} \mathfrak{u}=\mathfrak{f}$. In order to verify that the boundary conditions are satisfied by $\mathfrak{y}$, we set

$$
\gamma_{i}=\left(\mathfrak{f}^{1}, \underset{z a, i}{z}\right)+\left(\mathfrak{f}^{2}, \underset{z b, i}{2}\right), \quad i \in s_{1} .
$$

Then 


$$
\begin{aligned}
& \mathfrak{y}^{0}(a-)=\mathfrak{y}^{0}(a+)-\mathfrak{S}_{01} \mathfrak{y}^{1}=-\sum_{s_{1}} \gamma_{i}\left[\mathfrak{y}_{a, i}^{0}(a-)+\mathfrak{y}_{b, i}^{0}(a-)\right]-\mathfrak{S}_{01} \Re_{11}(\lambda) \mathfrak{f}^{1}, \\
& \mathfrak{y}^{0}(b+)=\mathfrak{y}^{0}(b-)+\mathfrak{S}_{02} \mathfrak{y}^{2}=-\sum_{s_{1}} \gamma_{i}\left[\mathfrak{y}_{a, i}^{0}(b+)+\mathfrak{y}_{b, i}^{0}(b+)\right]+\mathfrak{S}_{02} \Re_{22}(\lambda) \mathfrak{f}^{2} .
\end{aligned}
$$

For $j \in s_{1}$ we have

$$
\begin{aligned}
& -\left(\mathfrak{A}_{000} \mathfrak{y}^{0}, \stackrel{0}{z_{a, j}}\right)^{a-}+\left(\mathfrak{A}_{00} \mathfrak{y}^{0}, \stackrel{0}{z_{b}, j}\right)^{b+} \\
& =\sum_{\delta_{1}} \gamma_{i}\left[\left(\mathfrak{A}_{00}\left(\mathfrak{y}_{a, i}^{0}+\mathfrak{y}_{b, i}^{0}\right),{\stackrel{\mathfrak{z}}{z_{a, i}}}^{0}\right)^{a-}-\left(\mathfrak{A}_{00}\left(\mathfrak{y}_{a, i}^{0},+\mathfrak{y}_{b, i}^{0}\right), \stackrel{\mathfrak{z}}{b, i}^{0}\right)^{b+}\right] \\
& +\left(\mathfrak{A}_{00}\left(\mathfrak{S}_{01} \Re_{11}(\lambda) \mathfrak{f}^{1}, \underset{z_{a, i}}{0}\right)^{a-}-\left(\mathfrak{A}_{00} \mathfrak{S}_{02} R_{22}(\lambda) \mathfrak{f}^{2}, \underset{z b, i}{0}\right)^{b+} .\right.
\end{aligned}
$$

Now (3.18) and (7.28) combined give

$$
\begin{aligned}
& \left(\mathfrak{A}_{00} \mathfrak{y}_{a, i}^{0}, \stackrel{z}{z a}, j^{0}\right)^{a-}-\left(\mathfrak{A}_{00} \mathfrak{y}_{b, i}^{0}, \stackrel{z}{z} b, j^{0}\right)^{b+}=0, \\
& \left(\mathfrak{A}_{00}^{\mathfrak{y}_{b, i}, \mathfrak{z}_{a, j}^{0}}\right)^{a-}-\left(\mathfrak{A}_{00} \mathfrak{y}_{a, i}^{0}, \mathfrak{z}_{b, j}^{0}\right)^{b+}=\delta_{i j}, \quad \text { for all } i, j \in s_{1} \text {. }
\end{aligned}
$$

Hence for $j \in s_{1}$ the first sum in the right member of (7.35) is equal to simply $\gamma_{j}$. The second and third terms in the right member of (7.35) are handled differently. According to (7.9)

$$
\left(\mathfrak{A}_{00} \mathfrak{G}_{02} \Re_{22}(\lambda) \mathfrak{f}^{2}, \stackrel{0}{z_{b}, j}\right)^{b+}=\left(\Re_{22}(\lambda) \mathfrak{f}^{2}, \mathfrak{B}_{20 z_{b, j}^{0}}^{0}(b+)\right) .
$$

The analogue of $(7.23)$ for a solution $z l$ of $(7.19 \mathrm{a}, \mathrm{c})$ is

$$
\lambda_{z_{b}}^{2}-\mathfrak{B}_{22 z_{b}}^{*}-\mathfrak{B}_{20}^{0}\left(\mathfrak{S}_{02}^{0} z_{b}^{2} / 2=-\mathfrak{B}_{20 z_{b}}^{0}(b+) .\right.
$$

so that $z_{b}^{2}=-\Re_{22}(\lambda) * \mathfrak{B}_{20}^{0} z_{b}^{0}(b+)$. Consequently

$$
\left(\mathfrak { A } _ { 0 0 } \left(\mathfrak{S}_{02} \Re_{22}(\lambda) \mathfrak{f}^{2}, \underset{z_{b}, j}{b+}=-\left(\mathfrak{f}^{2}, \underset{z}{z} b, j\right)\right.\right.
$$

Similarly one shows that

$$
\left(\mathfrak{A}_{00} \mathfrak{S}_{01} \Re_{11}(\lambda) \mathfrak{f}^{1}, \stackrel{0}{z_{a}, j}\right)^{a-}=-\left(\mathfrak{f}^{1}, \mathfrak{z}_{a, j}^{1}\right)
$$

and it follows that

$$
-\left(\mathfrak{A}_{00} \mathfrak{Y}^{0}, \underset{z a, j}{0}\right)^{a-}+\left(\mathfrak{A}_{00} \mathfrak{Y}^{0}, \underset{z b, j}{0}\right)^{b+}=0
$$

for all $j \in s_{1}$. Now for $j \in s_{2}$ we have already noted that ${ }_{3 a, j}^{0} \equiv \theta$ so that ${ }_{3 a, j}^{1}=\theta$ and $z_{a, j}^{0}(a-)=z_{a, j}^{0}(a+)-\mathfrak{S}_{01}^{0} z_{a, j}^{1}=\theta$. By Lemma 5.6, $z_{b, j}^{0}(b-)=\theta$ so that $z_{b, j}^{2}=\theta$ and $z_{b, j}^{0}(b+)=z_{b, j}^{0}(b-)+\mathfrak{C}_{02}^{0} z_{b, j}^{2}=\theta$. Similarly for $j \in s_{3}$ we have $z_{b, j}^{0}(b+)=\theta$ and $z_{a, j}^{0}(a-)=\theta$. Consequently the condition (7.37) is trivially satisfied for $j \in s_{2} \cup s_{3}$. It therefore follows that $\mathfrak{y}=\Re(\lambda) \mathfrak{f} \in \mathfrak{D}(\Omega)$.

Combining the above two cases, we see that for any $\mathfrak{f} \in \mathfrak{S}$ we can define $\mathfrak{y}=\mathfrak{R}(\lambda) \mathfrak{f}$ in a suitable way so that $\mathfrak{y} \in \mathfrak{D}(\mathfrak{l})$ and $\lambda \mathfrak{y}-\mathfrak{l} \mathfrak{y}=\mathfrak{f}$. Moreover since $\mathfrak{y} \in \mathfrak{D}(\mathbb{R})$ the condition (7.32) will be satisfied. Actually (7.32) holds for ary $\mathfrak{y} \in \mathfrak{D}(\mathfrak{l})$ so that $\lambda \mathfrak{y}-\mathfrak{l} \mathfrak{y}=\mathfrak{f}$ has a unique solution and it follows from this by 
the usual argument that the range of $\Re(\lambda)$ fills out $\mathfrak{D}(\mathfrak{R})$. Together these facts show that $\Re(\lambda)=\Re(\lambda ; R)$.

Next we prove that $\mathfrak{D}(\mathfrak{R})$ is dense in $\mathfrak{S}$. Let $\mathfrak{f} \in \mathfrak{S}$ be given and choose any pair $\left[Y_{a}, Y_{b}\right] \in \mathfrak{N}_{a, b}$. Setting

$$
\mathfrak{y}^{0}(a+)=Y_{a}+\mathfrak{夭}_{01} \mathfrak{f}^{1} \text { and } \mathfrak{\eta}^{0}(b-)=Y_{b}-\mathfrak{夭}_{02} \mathfrak{f}^{2},
$$

it suffices to approximate $\mathfrak{f}^{0}$ in $\mathfrak{S}$ by an element $\mathfrak{y}^{0}$ of $\mathfrak{D}\left(L_{1}\right)$ with the above values of $\mathfrak{\eta}^{0}(a+)$ and $\mathfrak{y}^{0}(b-)$, in which case $\left(\mathfrak{f}^{1}, \mathfrak{y}^{0}, \mathfrak{f}^{2}\right)$ will provide the required approximation in $\mathfrak{D}(\mathfrak{R})$. Let $a^{\prime}, b^{\prime}$ be selected so that $a<a^{\prime}<b^{\prime}<b$ and

$$
\int_{a}^{a \prime}\left|\mathfrak{f}^{0}\right|^{2} d \xi+\int_{b^{\prime}}^{b}\left|\mathfrak{f}^{0}\right|^{2} d \xi<\epsilon^{2},
$$

and then approximate $f^{0}$ on $\left[a^{\prime}, b^{\prime}\right]$ in the mean to within $\epsilon$ by a smooth vector-valued function, say $\eta_{0}^{0}$ which vanishes outside of $\left[a^{\prime}, b^{\prime}\right]$. Further since $F_{a}$ spans $\mathfrak{Y}_{a}$ and $F_{b}$ spans $\mathfrak{Y}_{b}$ there is a $\mathfrak{y}_{a}^{0} \in F_{a}$ with $\mathfrak{y}_{a}^{0}(a+)=\mathfrak{y}^{0}(a+)$ and a $\mathfrak{y}_{b}^{0} \in F_{b}$ with $\mathfrak{\eta}_{b}^{0}(b-)=\mathfrak{\eta}^{0}(b-)$. Choose functions $\alpha$ and $\beta$ as in Lemma 4.2 so that they both vanish on $\left[a^{\prime}, b^{\prime}\right]$ and

$$
\int_{a}^{b}\left|\alpha \eta_{a}^{0}\right|^{2} d \xi+\int_{a}^{b}\left|\beta \eta_{b}^{0}\right|^{2} d \xi<\epsilon^{2} .
$$

Then $\mathfrak{y}^{0}=\alpha \eta_{a}^{0}+\mathfrak{\eta}_{0}^{0}+\beta \mathfrak{\eta}_{b}^{0}$ approximates $f^{0}$ in $L_{2}(a, b ; I)$ to within $5 \epsilon$. It follows that $\mathfrak{D}(\mathbb{R})$ is dense in $\mathfrak{S}$.

Thus all parts of the Hille-Yosida criterion have been verified for the operator $\mathfrak{\&}$ when $\lambda$ is sufficiently large. This being so, the Hille-Yosida theorem asserts that the criterion is actually satisfied for all $\lambda>0$.

A similar development, of course, holds for $\mathfrak{M}$. Further, it is easy to see that $\mathfrak{M} \subset \mathfrak{R}^{*}$; in fact for $\mathfrak{y \in D}(\mathfrak{R})$ and $z \in \mathfrak{D}(\mathfrak{M})$ we have by $(7.14)$

$$
\langle\mathfrak{R} \mathfrak{y}, \mathfrak{z}\rangle-\left\langle\mathfrak{y}, \mathfrak{M}_{\mathfrak{z}}\right\rangle=-\left(\mathfrak{A}_{00} \mathfrak{y}^{0}, \mathfrak{z}^{0}\right)^{a-}+\left(\mathfrak{A}_{00}, \mathfrak{z}^{0}\right)^{b+}=0 .
$$

On the other hand both $\Re(\lambda ; \mathfrak{R})^{*}=\Re\left(\lambda ; \mathfrak{R}^{*}\right)$ and $\Re(\lambda ; \mathfrak{M})$ exist for $\lambda>0$ so that both $\lambda I-\Omega^{*}$ and $\lambda I-\mathfrak{M}$ map their respective domains in a one-to-one fashion onto $\mathfrak{S}$. This requires $\mathfrak{D}\left(\mathfrak{R}^{*}\right)=\mathfrak{D}(\mathfrak{M})$, that is, $\mathfrak{R}^{*}=\mathfrak{M}$. The analogous argument shows that $\mathfrak{M}^{*}=\mathbb{R}$. This concludes the proof of Theorem 7.1.

Using the representation (7.30) it is easy to compute $\Re(\lambda ; \mathfrak{M})=\Re\left(\lambda ; \Omega^{*}\right)$ directly as $\Re(\lambda ; R)^{*}$. We have, in fact

$$
\begin{aligned}
& {[\Re(\lambda ; \mathfrak{R}) \mathfrak{g}]^{0}=\Re_{00}(\lambda)^{*} \mathfrak{g}^{0}-\sum_{s_{1} \cup}{ }_{z_{b,}}^{0}{ }_{b, i}\left(\mathfrak{g}^{\prime},\left(\mathfrak{y}_{a, i}^{1}+\mathfrak{y}_{b, i}^{1}\right)\right)} \\
& -\sum_{s_{1} \cup_{s_{3}}} z_{a, i}^{0}\left(g^{2},\left(\mathfrak{y}_{a, i}^{2}+\mathfrak{y}_{l, i}^{2}\right)\right) \text {, } \\
& {[\mathfrak{R}(\lambda ; \mathfrak{M}) \mathfrak{g}]^{1}=\Re_{11}(\lambda)^{*} \mathfrak{g}^{1}-\sum_{s_{1}} \mathfrak{z}_{a, i}^{1}\left\langle\mathfrak{g},\left(\mathfrak{y}_{a, i}+\mathfrak{y}_{l, i}\right)\right\rangle,} \\
& {[\Re(\lambda ; \mathfrak{M}) \mathfrak{g}]^{2}=\Re_{22}(\lambda)^{*} \mathfrak{g}^{2}-\sum_{s_{1}} \underset{z_{b, i}}{2}\left\langle g,\left(\mathfrak{y}_{a, i}+\mathfrak{y}_{l, i}\right)\right\rangle}
\end{aligned}
$$


where $\left({ }^{11}\right)$

$$
\begin{aligned}
& R_{00}(\lambda)^{*} g^{0}=\left(\begin{array}{c}
\Re_{00}(\lambda)^{*}\left[g^{0}\right]^{0} \\
\theta
\end{array}\right)-\sum_{,_{1} \cup_{a b}} z_{a, i}^{0} \int_{x}^{b}\left(g^{0},\left(y_{a, i}^{0}+y_{b, i}^{0}\right)\right) d \xi \\
& -\sum_{i_{1} \cup} \mathfrak{z}_{b, i}^{0} \int_{a}^{x}\left(\mathfrak{g}^{0},\left(\mathfrak{y}_{a, i}^{0}+\mathfrak{y}_{b, i}^{0}\right)\right) d \xi \text {. }
\end{aligned}
$$

The sense in which $\Re_{00}(\lambda){ }^{*} g^{0}$ is to be taken has already been made clear in Theorem 4.1. The other terms appearing in this expression for $\mathfrak{R}(\lambda ; \mathfrak{M})$ are all well defined.

As a converse to Theorem 7.1, we have

THEOREM 7.2. Every dissipative restriction of $\mathfrak{R}_{1}$ (or of $\mathfrak{M}_{1}$ ) which generates a strongly continuous semi-group of linear bounded operators is of the type described in Theorem 7.1 .

The proof of this assertion paraphrases that of Theorem 6.2 and is omitted.

It is of interest at this point to consider the connection between a semigroup solution to the initial value problem and a solution in the classical sense. Let $\mathfrak{f}_{0} \in \mathfrak{D}\left(\mathbb{R}^{2}\right)$. Then $\mathfrak{f}_{0}$ can be expressed as $\mathfrak{f}_{0}=\mathfrak{R}(\lambda ; \mathfrak{l}) \mathfrak{f}_{1}$ for some $\mathfrak{f}_{1} \in \mathfrak{D}(\mathfrak{R})$ and fixed $\lambda>0$. As a consequence, if $\mathbb{R}$ generates the semi-group $[\Im(t) ; t \geqq 0]$, then

$$
\mathfrak{y}(t) \equiv \mathfrak{S}(t) \mathfrak{f}_{0}=\Im(t) \Re(\lambda ; \mathfrak{R}) \mathfrak{f}_{1}=\mathfrak{R}(\lambda ; \mathfrak{R}) \Im(t) \mathfrak{f}_{1}
$$

We now avail ourselves of the representation (7.30) for $\Re(\lambda ; \mathfrak{l}), \mathfrak{R}_{00}(\lambda ; \mathfrak{l})$ being given by (4.23), and by means of this we can write $\mathfrak{y}^{0}(t)$ explicitly as a vector function of $x_{-}$for each $t$, namely,

$$
\mathfrak{y}^{0}(t, x)=\left[\Re(\lambda ; \mathfrak{R}) \subseteq(t) \mathfrak{f}_{1}\right]^{0}(x) .
$$

Actually this only determines $\left[\mathfrak{y}^{0}(t)\right]^{1}$ explicitly as a function of $x$ and $t$, since for each $t,\left[\mathfrak{y}^{0}(t)\right]^{0}$ is determined by this expression only to within a set of measure zero $\left({ }^{11}\right)$. However $[\mathfrak{y}(t)]^{0}$ is in any case two times strongly continuously differentiable $\left(\mathfrak{f}_{0} \in \mathfrak{D}\left(\mathfrak{Q}^{2}\right)\right)$ so we can appeal to a theorem in Hille and Phillips [7, Theorem 3.4.2] which asserts that there exists a pointwise representation of $\left[\mathfrak{y}^{0}(t)\right]^{0}$ which is continuously differentiable in $t$ for each $x$ and measurable in the $(x, t)$ space. We denote this representation of $\left[\mathfrak{y}^{0}(t)\right]^{0}$ by $\left[\mathfrak{y}^{0}(t, x)\right]^{0}$ and this together with the above defined $\left[\mathfrak{y}^{0}(t, x)\right]^{1}$ now determines $\mathfrak{y}^{0}(t)$ as a function of $x$ and $t$. Making use of the strong differentiability of $\mathfrak{S}(t) \mathfrak{f}_{1}$, it is readily seen that the 1 -component of $\mathfrak{y}^{0}(t, x)$ is continuously differentiable in $t$ for each $x$; in fact,

(11) The outer superscript 0 or 1 refers to the notation introduced in (2.18). 


$$
\begin{aligned}
\frac{\partial y^{0}(t, x)}{\partial t} & =\left[\Re(\lambda ; \mathfrak{R}) \frac{d \subseteq(t) \mathfrak{f}_{1}}{d t}\right]^{0}(x)=\left[\Re(\lambda ; \mathfrak{R}) \mathfrak{R} \subseteq(t) \mathfrak{f}_{1}\right]^{0}(x) \\
& =\left[\mathfrak{R N}(\lambda ; \mathfrak{R}) \subseteq(t) \mathfrak{f}_{1}\right]^{0}(x)=[\mathbb{R}(t)]^{0}(x) .
\end{aligned}
$$

The only advantage in using the resolvent to define $\left[y^{0}(t, x)\right]^{1}$ over the above mentioned Hille-Phillips theorem is that the resolvent furnishes a realization which is absolutely continuous in $x$ for each $t$; thus $\mathfrak{y}^{0}(t, x)$ is actually in $\mathfrak{D}\left(L_{1}\right)$ and not simply equal to a function in $\mathfrak{D}\left(L_{1}\right)$ almost everywhere.

8. Conservative systems. We shall speak of an operator $\ell_{1}$ as being conservative if no energy is dissipated internally, that is, if

$$
\mathfrak{B}+\mathfrak{B}^{*}+\mathfrak{A}_{x}=\Theta, \quad a<x<b .
$$

We note that in this case $\mathfrak{I}_{00}=I$. An operator $\mathfrak{R}$ will be called a conservative restriction of $\ell_{1}$ if no energy is lost through the boundary, that is, if

$$
-\left(\mathfrak{A}_{00} \mathfrak{y}^{0}, \mathfrak{y}^{0}\right)^{a-}+\left(\mathfrak{A}_{00} \mathfrak{y}^{0}, \mathfrak{y}^{0}\right)^{b+}=0
$$

for all $\mathfrak{n} \in \mathfrak{D}(\mathfrak{R})$.

THEOREM 8.1. If $\&$ is a conservative restriction of the conservative operator $\Omega_{1}$ satisfying the Hille-Yosida criterion, then $\&$ generates a semi-group of isometries.

Proof. Let $[\varsigma(t) ; t \geqq 0]$ be the semi-group of operators generated by $\mathbb{Z}$ and suppose that $\mathfrak{y} \in \mathfrak{D}(\mathfrak{R})$. Then $\mathfrak{S}(t) \mathfrak{y} \in \mathfrak{D}(\mathfrak{R})$ for all $t \geqq 0$ and

$$
\frac{d}{d t}\langle\mathfrak{S}(t) \mathfrak{y}, \mathfrak{S}(t) \mathfrak{y}\rangle=\langle\mathfrak{I} S(t) \mathfrak{y}, \mathfrak{S}(t) \mathfrak{y}\rangle+\langle\mathfrak{S}(t) \mathfrak{y}, \mathfrak{R} S(t) \mathfrak{y}\rangle, \quad t \geqq 0 .
$$

It follows from (7.14) together with (8.1) and (8.2) that $d / d t\langle\subseteq(t) \mathfrak{y}, \subseteq(t) \mathfrak{y}\rangle=\theta$ for all $t \geqq 0$ so that $\|\mathfrak{S}(t) \mathfrak{y}\|=\|\mathfrak{y}\|, t \geqq 0$. The domain $\mathfrak{D}(\mathfrak{l})$ being dense in $\mathfrak{S}$, we see that this holds for all $\mathfrak{n} \in \mathfrak{S}$ and therefore $\subseteq(t)$ is an isometry.

The above result is not surprising. One might even expect such a conservative restriction of $\mathfrak{R}_{1}$ to generate a group so that the resulting process is reversible in time. However this is not the case as the following simple example shows: Let $H=L_{2}(a, b)$ with $a=0, b=\infty$, and define

$$
L_{1} y=-y_{x} \text { with } \mathfrak{D}\left(L_{1}\right)=\left[y ; y \text { absolutely continuous, } y \text { and } y_{x} \in H\right] \text {. }
$$

Then

$$
M_{1} z=z_{x} \text { with } \mathfrak{D}\left(M_{1}\right)=\left[z ; z \text { absolutely continuous, } z \text { and } z_{x} \in H\right] .
$$

It is readily verified that $n=1, p=0, r=1, l_{a}=1=m_{a}, l_{b}=1$, and $m_{b}=0$. Thus $d_{a}=1$ and $d_{b}=0$. Consequently for $y \in \mathfrak{D}\left(L_{1}\right)$,

$$
-(A y, y)^{a}+(A y, y)^{b}=-(A y, y)^{a}=|y(0)|^{2},
$$

and the only dissipative boundary condition is $y(0)=0$, which is also a con- 
servative boundary condition. The corresponding restriction $L$ generates the semi-group of operators

$$
[S(t) f](x)=f(x-t) .
$$

It is clear that the range of $S(t)$ fills out $L_{2}(t, \infty)$ but not $L_{2}(0, \infty)$ for $t>0$. Consequently $S(t)$ does not have an inverse and $L$ does not generate a group.

Evidently, then, further conditions are required on a conservative restriction in order that it generate a group of operators. We note that the condition (8.1) suffices to make $R_{1}=-\mathfrak{M}_{1}$ so that $i R_{1}$ is formally symmetric. This suggests the following result.

THEOREM 8.2. A conservative restriction $\mathbb{R}$ of a conservative operator $\mathfrak{R}_{1}$ generates a group if and only if is if self-adjoint. In this case the group generated by $\mathbb{R}$ consists of unitary operators.

Proof. If $\&$ is a conservative restriction and generates a group of operators $[\subseteq(t) ;-\infty<t<\infty]$, then $\subseteq(t)^{-1}=\subseteq(-t)$ exists and by the previous theorem $\mathfrak{S}(t)$ is an isometry for $t>0$. It follows from these two facts that $\subseteq(t)$ is unitary for all $t$. The rest of the assertion of the theorem is well known (see J. von Neumann [11]); however, for the sake of completeness, we shall include an independent proof. Suppose first that $\&$ generates a group of unitary operators. Then for all $\mathfrak{y} \in \mathfrak{D}(\mathfrak{l})$ we have $d \mathfrak{S}(t) \mathfrak{n} /\left.d t\right|_{t=0}=\mathfrak{l} \mathfrak{y}$. Since $\langle\mathfrak{S}(t) \mathfrak{y}, \mathfrak{u}\rangle$ $=\left\langle\mathfrak{y}, \mathfrak{S}^{*}(t) \mathfrak{u}\right\rangle=\langle\mathfrak{y}, \mathfrak{S}(-t) \mathfrak{u}\rangle$, it follows on differentiating that $\langle\mathfrak{l} \mathfrak{y}, \mathfrak{u}\rangle=-\langle\mathfrak{y}, \mathfrak{R} \mathfrak{u}\rangle$ for all $\mathfrak{y}, \mathfrak{u} \in \mathfrak{D}(\mathfrak{R})$; thus $i \mathfrak{R}$ is symmetric. On the other hand $\mathfrak{R}$ generates a group of unitary operators and this implies (see Hille and Phillips [7, Theorem 1.2.3.2]) that $\Re(\lambda ; \mathfrak{R})$ exists for $\lambda= \pm 1$. Thus the deficiency indices are zero and as a consequence $i \ell$ is self-adjoint. Conversely, if $i \ell$ is self-adjoint, then one can show directly that $\|\lambda \Re(\lambda ; \mathfrak{R})\| \leqq 1$ for all real $\lambda \neq 0$. The above quoted Hille-Phillips theorem now implies that $\&$ generates a group.

We next determine necessary and sufficient conditions for a conservative operator $\ell_{1}$ that $i \ell_{1}$ have a self-adjoint restriction. For this purpose it is convenient to introduce the restriction $\Omega_{0} \subset \Omega_{1}$ defined by

$$
\mathfrak{D}\left(\mathfrak{R}_{0}\right)=\left[\mathfrak{y} ; \mathfrak{y} \in \mathfrak{D}\left(\mathfrak{R}_{1}\right), \quad \mathfrak{y}^{0}(a-)=\theta=\mathfrak{y}^{0}(b+)\right] .
$$

For $\mathfrak{h} \in \mathfrak{D}\left(\mathfrak{R}_{0}\right)$ and $\mathfrak{u} \in \mathfrak{D}\left(\mathfrak{R}_{1}\right)$ we have by $(7.14)$

$$
\left\langle\mathfrak{R}_{0} \mathfrak{y}, \mathfrak{u}\right\rangle+\left\langle\mathfrak{y}, \mathfrak{R}_{1} \mathfrak{u}\right\rangle=-\left(\mathfrak{A}_{00} \mathfrak{l}^{0}, \mathfrak{u}^{0}\right)^{a-}+\left(\mathfrak{A}_{000} \mathfrak{y}^{0}, \mathfrak{u}^{0}\right)^{b+}=0,
$$

from which it follows that $i \Omega_{0}$ is symmetric. Actually (8.4) implies more, namely that $i \Omega_{0} \subset\left(i \Omega_{1}\right)^{*}$ and as a consequence any self adjoint restriction of $i \Omega_{1}$ necessarily contains $i \Omega_{0}$. This suggests that we consider the self-adjoint extensions of $i \Omega_{0}$ rather than the self-adjoint restrictions of $i \Omega_{1}$; it will turn out that we obtain the same class of self-adjoint operators in either case. Now according to the Cayley transform theory (see J. von Neumann [10]), the operator $i \Omega_{0}$ has a self-adjoint extension if and only if its deficiency indices are equal. If we define $\mathfrak{M}_{0} \subset \mathfrak{M}_{1}$ by 


$$
\mathfrak{D}\left(\mathfrak{M}_{0}\right)=\left[z ; z \in \mathfrak{D}\left(\mathfrak{M}_{1}\right), z^{0}(a-)=\theta=z^{0}(b+)\right],
$$

then clearly $\mathfrak{M}_{0}=-\mathfrak{R}_{0}$ and the above criterion for $i \mathfrak{R}_{0}$ to have a self-adjoint extension is simply the assertion that the subspaces

$$
\mathfrak{S} \theta \text { [range of } \lambda I-\mathfrak{R}_{0} \text { ] and } \mathfrak{S} \theta \text { [range of } \lambda I-\mathfrak{M}_{0} \text { ] }
$$

be of equal dimension for some $\lambda>0$. The following theorem, which gives a method for determining the dimension of the range of $\lambda I-\mathfrak{M}_{0}$, does not require that the operator $\mathfrak{R}_{1}$ be conservative.

THEOREM 8.3. Let

$$
\mathfrak{U}_{\lambda}=\left[\mathfrak{y} ; \mathfrak{y} \in \mathfrak{D}\left(\mathfrak{R}_{1}\right), \lambda \mathfrak{y}-\mathfrak{R}_{1} \mathfrak{y}=\theta\right] .
$$

If $\lambda$ is not a characteristic value of the matrices (7.21) and (7.24), then

$$
\mathfrak{U}_{\lambda}=\mathfrak{S} \theta\left[\text { range of } \lambda I-\mathfrak{M}_{0}\right] .
$$

Proof. It is easy to see that $\mathfrak{U}_{\lambda} \subset \mathfrak{S} \theta$ [range of $\lambda I-\mathfrak{M}_{0}$ ]. In fact for $\mathfrak{y} \in \mathfrak{U}_{\lambda}$ and $z \in \mathfrak{D}\left(\mathfrak{M}_{0}\right)$ we have by $(7.14)$

$$
\left\langle\mathfrak{y},\left(\lambda_{z}-\mathfrak{M}_{0 z}\right)\right\rangle=\left\langle\left(\lambda \mathfrak{y}-\mathfrak{R}_{1} \mathfrak{y}\right), \mathfrak{z}^{\mathfrak{z}}\right\rangle-\left(\mathfrak{A}_{00 \mathfrak{y}^{0}}, \mathfrak{z}^{0}\right)^{a-}+\left(\mathfrak{I}_{00} \mathfrak{y}^{0}, z^{0}\right)^{b+}=0 .
$$

To establish the inequality in the other direction we suppose that $\mathfrak{U}_{\lambda}$ does not fill out $\mathfrak{E} \theta$ [range of $\lambda I-\mathfrak{M}_{0}$ ]. Then there exists a nontrivial $\mathfrak{g} \in \mathfrak{S} \ominus$ [range of $\lambda I-\mathfrak{M}_{0}$ ] which is orthogonal to $\mathfrak{U}_{\lambda}$. Now it is clear from (8.5) that any dissipative restriction $\mathfrak{M} \subset \mathfrak{M}_{1}$ of the type considered in Theorem 7.1 will contain $\mathfrak{M}_{0}$. Since the range of $\lambda I-\mathfrak{M}$ coincides with $\mathfrak{E}$, there is a $z \in \mathfrak{D}(\mathfrak{M})$ with $\lambda_{z}-\mathfrak{M}_{\mathfrak{z}}=\mathfrak{g}$; obviously $z \in \mathfrak{D}\left(\mathfrak{M}_{0}\right)$. Thus $z=\mathfrak{R}(\lambda ; \mathfrak{R}) \mathfrak{g}$ and $\mathfrak{g} \in \mathfrak{S} \ominus \mathfrak{U}_{\lambda}$. We now show that these two conditions imply that $z \in \mathfrak{D}\left(\mathfrak{M}_{0}\right)$, contrary to our choice of $z$. It suffices to show that

$$
z^{0}(a+)=\mathfrak{G}_{013}^{0}{ }^{1} \text { and } \mathfrak{z}^{0}(b-)=-\mathfrak{G}_{02 z^{2}}^{0},
$$

since it follows from this and (7.13) that $z^{\circ}(a-)=\theta$ and $z^{\circ}(b+)=\theta$ and hence that $z \in \mathfrak{D}\left(\mathfrak{M}_{0}\right)$. We shall use the representation (7.38) for $\mathfrak{R}(\lambda ; \mathfrak{M})$ to establish (8.7).

It will be recalled that the solution pairs $\left[\mathfrak{y}_{a, i}^{0}, \mathfrak{y}_{b, i}^{0}\right]$ in (7.38) were chosen to be linearly independent. We now show that the solutions $\left[\left(\mathfrak{\eta}_{a, i}^{0}+\mathfrak{\eta}_{b, i}^{0}\right)\right.$; $i=1,2, \cdots, r]$ are also linearly independent. In fact, if there did exist constants $\nu_{i}$, not all zero, such that $\sum \nu_{i}\left(\mathfrak{y}_{a, i}^{0}+\mathfrak{y}_{b, i}^{0}\right)=\theta$ for some $x_{0} \in(a, b)$, then writing

$$
\mathfrak{y}_{a} \equiv \sum \nu_{i} \mathfrak{y}_{a, i} \quad \text { and } \quad \mathfrak{y}_{b} \equiv \sum-\nu_{i} \mathfrak{y}_{b, i}
$$

we have $\mathfrak{y}_{a}^{0}\left(x_{0}\right)=\mathfrak{y}_{b}^{0}\left(x_{0}\right)$ and $-\left(\mathfrak{A}_{00} \mathfrak{y}_{a}^{0}, \mathfrak{y}_{a}^{0}\right)^{a-}+\left(\mathfrak{A}_{00} \mathfrak{y}_{b}^{0}, \mathfrak{y}_{b}^{0}\right)^{b+} \leqq 0$; and Lemma 7.1 implies that $\mathfrak{y}_{a}^{0} \equiv \mathfrak{y}_{b}^{0}$. Thus $\sum \nu_{i}\left[\mathfrak{y}_{a, i}, \mathfrak{y}_{b, i}\right] \equiv[\theta, \theta]$, which is impossible. Since there are just $r$ linearly independent solutions of (3.1) by Theorem 3.1, it follows that the solutions $\left[\left(\mathfrak{y}_{a, i}^{0}+\mathfrak{y}_{b, i}^{0}\right) ; i=1,2, \cdots, r\right]$ span the solution space. 
Further by our selection of the bases, the $\left[\left(\mathfrak{y}_{a, i}+\mathfrak{y}_{b, i}\right) ; i \in s_{1}\right]$ span $\mathfrak{u}_{\lambda}$, the $\left[\left(\mathfrak{\eta}_{a, i}^{0}+\mathfrak{y}_{b, i}^{0}\right) ; i \in s_{1} \cup s_{2}\right]$ span $F_{a}$, and the $\left[\left(\mathfrak{\eta}_{a, i}^{0}+\mathfrak{\eta}_{b, i}^{0}\right) ; i \in s_{1} \cup s_{3}\right] \operatorname{span} F_{b}$.

Returning now to the above $z \in \mathfrak{D}(\mathfrak{M})$ we see that $\left\langle g,\left(\mathfrak{y}_{a, i}+\mathfrak{y}_{b, i}\right)\right\rangle=0$ for $i \in s_{1}$ and hence by the representation (7.38) we have

$$
z^{1}=\Re_{11}(\lambda)^{*} g^{1} \text { and } z^{2}=\Re_{22}(\lambda)^{*} g^{2} .
$$

Also it is clear from (7.38) that if $\mathfrak{g}^{0}$ vanishes near $b$ and if $\eta_{b}$ is a solution of $(7.18 \mathrm{a}, \mathrm{c})$ with $\mathfrak{y}_{b}^{0} \in F_{b}$, then

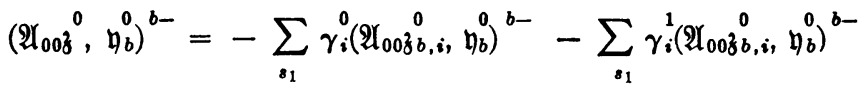

$$
\begin{aligned}
& -\sum_{s_{1} \bigcup_{s_{3}}} \gamma_{i}^{2}\left(\mathfrak{A}_{00 z_{a, i}, \mathfrak{y}_{b}^{0}}^{0}\right)^{b-},
\end{aligned}
$$

where

$$
\begin{gathered}
\gamma_{i}^{0}=\int_{a}^{b}\left(\mathfrak{g},\left(\mathfrak{y}_{a, i}^{0}+\mathfrak{y}_{b, i}^{0}\right)\right) d x, \\
\gamma_{i}^{1}=\left(\mathfrak{g}^{1},\left(\mathfrak{y}_{a, i}^{1}+\mathfrak{y}_{b, i}^{1}\right)\right), \quad \gamma_{i}^{2}=\left(\mathfrak{g}^{2},\left(\mathfrak{y}_{a, i}^{2}+\mathfrak{y}_{b, i}^{2}\right)\right) ;
\end{gathered}
$$

the first two sums in the right member of (8.9) extend only over $s_{1}$ because of Lemma 5.6. For the above $z \in \mathfrak{D}(\mathfrak{M})$ with $\mathfrak{g}=\lambda_{z}-\mathfrak{M}_{z}$, we can approximate $\mathfrak{g}$ in $\mathfrak{S}$ by a sequence $\left\{\mathfrak{g}_{n}\right\}$ such that each of the $\mathfrak{g}_{n}^{0}$ s vanishes near $b$. We see that $\gamma_{i, n}^{k} \rightarrow \gamma_{i}^{k}$ as $n \rightarrow \infty$ for $i \in s_{1}$ when $k=0$, for $i \in s_{1} \cup s_{2}$ when $k=1$, and for $i \in s_{1} \cup s_{3}$ when $k=2$. Further applying Lemma 4.3 to the sequence $\left\{z_{n}^{0}=\left[\mathfrak{R}(\lambda ; \mathfrak{M}) \mathfrak{g}_{n}\right]^{0}\right\}$ we see that $\left(\mathfrak{A}_{00 z_{n}^{0}}, \mathfrak{y}_{b}^{0}\right)^{b-} \rightarrow\left(\mathfrak{A}_{00 z^{0}}, \mathfrak{y}_{b}^{0}\right)^{b-}$. As a consequence (8.9) holds for the $z \in \mathfrak{D}(\mathfrak{M})$ which we are considering. Recalling that $z_{b, i}^{0} \equiv \theta$ for $i \in s_{3}$, we see that (8.9) can be written as

$$
\begin{aligned}
& \left(\mathfrak{A}_{00 z^{0}}, \mathfrak{y}_{b}^{0}\right)^{b-}=-\sum_{s_{1}} \gamma_{i}\left(\mathfrak{A}_{00 b_{b, i}, \mathfrak{y}_{b}^{0}}^{0}\right)^{b-}
\end{aligned}
$$

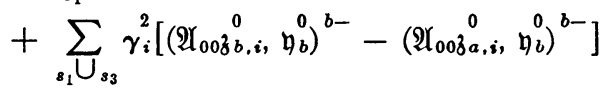

where $\gamma_{i}=\gamma_{i}^{0}+\gamma_{i}^{1}+\gamma_{i}^{2}=\left\langle\mathfrak{g},\left(\mathfrak{y}_{a, i}+\mathfrak{y}_{b, i}\right)\right\rangle$. As we noted above, for our choice of z we have $\gamma_{i}=0$ for $i \in s_{1}$. Hence replacing $\mathfrak{y}_{b}$ in $(8.10)$ by $\left(\mathfrak{y}_{a, j}+\mathfrak{y}_{b, j}\right), j \in s_{1} \cup s_{3}$, and making use of the identities (7.36) which hold for all $i, j$ if we replace $a-$ and $b+$ by $b-$ (as can be seen directly from (3.18)), we finally obtain

$$
\left(\mathfrak{A}_{003}^{0},\left(\mathfrak{y}_{a, j}^{0}+\mathfrak{y}_{b, j}^{0}\right)\right)^{b-}=-\gamma_{j}^{2}, \quad j \in s_{1} \cup s_{3} .
$$

Now for $\mathfrak{y}_{b}$ a solution of $(7.18 \mathrm{a}, \mathrm{c})$ we can make use of (7.31) to write

$$
\left(\mathfrak{g}^{2}, \mathfrak{y}_{b}^{2}\right)=\left(\Re_{22}(\lambda) * \mathfrak{g}^{2},\left(\lambda \mathfrak{y}_{b}^{2}-\mathfrak{B}_{22} \mathfrak{y}_{b}^{2}-\mathfrak{B}_{20} \mathfrak{E}_{02} \mathfrak{y}_{b}^{2} / 2\right)\right)
$$

and with the aid of (7.20), (8.8) and (7.9) this becomes 


$$
\left(\mathfrak{g}_{2}, \mathfrak{y}_{b}^{2}\right)=\left(\mathfrak{3}^{2}, \mathfrak{B}_{20} \mathfrak{y}_{b}^{0}(b-)\right)=\left(\mathfrak{A}_{00} \mathfrak{S}_{02 \mathfrak{z}}^{0}, \mathfrak{y}_{b}^{0}\right)^{b-} .
$$

Thus (8.11) gives

$$
\left(\mathfrak{R}_{00 z}^{0},\left(\mathfrak{y}_{a, j}^{0}+\mathfrak{y}_{b, j}^{0}\right)\right)^{b-}=-\left(\mathfrak{A}_{00} \mathfrak{C S}_{02 \mathfrak{z}}^{0},\left(\mathfrak{y}_{a, j}^{0}+\mathfrak{\eta}_{b, j}^{0}\right)\right)^{b-}
$$

for all $j \in s_{1} \cup s_{3}$. In view of the fact that the $\left[\left(\mathfrak{y}_{a, j}^{0}(b-)+\mathfrak{y}_{b, j}^{0}(b-)\right) ; j \in s_{1} \cup s_{3}\right]$ span $\mathfrak{Y}_{b}$, this implies $z^{0}(b-)=-\mathfrak{C}_{02}^{0} z^{2}$. The first relation in (8.7) is proved similarly. As a consequence $z \in \mathfrak{I}\left(\mathfrak{M}_{0}\right)$ and hence our original assumption, namely that $\mathfrak{U}_{\lambda}$ does not fill out $\mathfrak{S} \theta$ [range of $\lambda I-\mathfrak{M}_{0}$ ], was false.

To complete this discussion we next prove

THEOREM 8.4. If $\mathfrak{R}_{1}$ is a conservative operator and the deficiency indices of $i \Omega_{0}$ are equal, then $i \Omega_{0}$ has self-adjoint extensions, all such extensions are conservative restrictions of $i \Omega_{1}$, and, conversely, each conservative restriction of $i \Omega_{1}$ which generates a semi-group is a self-adjoint extension of $i \Omega_{0}$.

Proof. If the deficiency indices of $i \Omega_{0}$ are equal, then there exist self-adjoint extensions. In fact, according to the Cayley transform theory all such extensions are obtained in the following fashion: For fixed (sufficiently large) $\lambda>0$ let $\mathfrak{U}_{\lambda}$ be the solution space of $\lambda \mathfrak{u}-\mathfrak{R}_{1} \mathfrak{u}=\theta$ and let $\mathfrak{B}_{\lambda}$ be the solution space of $\lambda \mathfrak{b}+R_{1} \mathfrak{v} \equiv \lambda \mathfrak{v}-\mathfrak{M}_{1} \mathfrak{v}=\theta$. The previous theorem asserts that

$$
\mathfrak{l l}_{\lambda}=\mathfrak{S} \theta\left[\text { range of } \lambda I-\mathfrak{M}_{0}\right] \text { and } \mathfrak{B}_{\lambda}=\mathfrak{S} \theta\left[\text { range of } \lambda I-\mathfrak{R}_{0}\right],
$$

and since the deficiency indices are equal the subspaces $\mathfrak{U}_{\lambda}$ and $\mathfrak{B}_{\lambda}$ will be of equal dimension. Finally let $\mathfrak{B}$ be an arbitrary isometry with domain $\mathfrak{B}_{\lambda}$ and range $\mathfrak{l}_{\lambda}$. Then each self-adjoint extension $i \ell$ of $i \mathbb{R}_{0}$ is defined by such an isometry as

$$
\begin{aligned}
\mathfrak{I}(\mathfrak{l}) & =\left[\mathfrak{y} ; \mathfrak{y}=\mathfrak{y}_{1}+\mathfrak{y}_{2}, \mathfrak{y}_{1} \in \mathfrak{D}\left(\mathfrak{R}_{0}\right), \mathfrak{y}_{2}=\mathfrak{v}+\mathfrak{W} \mathfrak{b} \text { with } \mathfrak{v} \in \mathfrak{B}_{\lambda}\right], \\
\mathfrak{l} \mathfrak{y} & =\mathfrak{R}_{0} \mathfrak{y}_{1}+\lambda(\mathfrak{W} \mathfrak{v}-\mathfrak{v}) .
\end{aligned}
$$

Now $\mathfrak{R}_{1}=-\mathfrak{M}_{1}$ so that $\mathfrak{U}_{\lambda} \cup \mathfrak{B}_{\lambda} \subset \mathfrak{D}\left(\mathfrak{R}_{1}\right)$; consequently $\mathfrak{D}(\mathfrak{R}) \subset \mathfrak{D}\left(\mathfrak{R}_{1}\right)$. Finally since $i \Omega$ is a self-adjoint restriction of $i \Omega_{1}$ we have by (7.14)

$$
0=\langle\mathfrak{l} \mathfrak{y}, \mathfrak{y}\rangle+\langle\mathfrak{y}, \mathfrak{l} \mathfrak{y}\rangle=-\left(\mathfrak{A}_{00} \mathfrak{y}^{0}, \mathfrak{y}^{0}\right)^{a-}+\left(\mathfrak{P}_{00} \mathfrak{y}^{0}, \mathfrak{y}^{0}\right)^{b+}
$$

which proves that $\mathfrak{R}$ is a conservative restriction of $\ell_{1}$. Conversely, suppose that $\mathbb{R}$ is a conservative restriction of $R_{1}$ generating a semi-group of operators so that $\mathfrak{D}(\mathbb{R})$ is of the type (7.29). It then follows from (8.3) that $\mathbb{R}_{0} \subset \mathbb{R}$. Thus $i \Omega$ is a symmetric extension of the operator $i \Omega_{0}$ which has finite and equal deficiency indices and the only such extensions are self-adjoint.

REMARK. For a conservative restriction $\mathfrak{l}$ of a conservative operator $\mathfrak{l}_{1}$, the operator $i \&$ is always self-adjoint in the two extreme cases, namely when the system is so regular that all solutions of (7.18) and (7.19) lie in $\mathfrak{S}$ or when 
the system is so singular that none of the solutions of (7.18) and (7.19) lie in $\mathfrak{W}$. In the latter case the deficiency indices are both zero and the operator $i \mathbb{R}_{0}$ is itself self-adjoint; in fact this case is so degenerate that $\mathbb{R}_{1}=\mathbb{R}_{0}\left({ }^{12}\right)$.

Before concluding this section, we return to the telegraphist equation (1.3). We see by (1.5) that the corresponding system operator is conservative if and only if $r(x) \equiv 0$. In this case $t$ enters in (1.3) only as a second derivative so that if $u(t, x)$ is a solution then so is $u(-t, x)$. Moreover, since the boundary conditions are homogeneous of degree one in $u_{t}$, the boundary conditions will also be satisfied by $u(-t, x)$ if they are satisfied by $u(t, x)$. Basically this is what is required of a system to be reversible. It is somewhat surprising, therefore, that our criteria for a conservative operator to generate a group cannot be verified by inspection for the corresponding system defined by the matrices (1.4). We shall now bring this system into a form in which the group property becomes evident. This will be accomplished by means of three successive transformations, the first of which, taking $E$ into $E^{\prime} \equiv I$, is defined by

$$
V(x)=[E(x)]^{-1 / 2}=\left(\begin{array}{ccc}
s^{-1 / 2} & 0 & 0 \\
0 & p^{-1 / 2} & 0 \\
0 & 0 & q^{-1 / 2}
\end{array}\right) \text {. }
$$

We then make a change of independent variable (see (2.20))

$$
\xi(x)=\int_{c}^{x}[\rho(\sigma)]^{2} d \sigma, \quad \rho(x)=[q(x)]^{1 / 2} ;
$$

and this is followed by another transformation of the type (2.1) with

$$
V(x)=\left(\begin{array}{ccc}
1 & 0 & 0 \\
0 & 2^{-1 / 2} & 2^{-1 / 2} \\
0 & -2^{-1 / 2} & 2^{-1 / 2}
\end{array}\right)
$$

The result of these three transformations is to bring the system into the form

$$
E=I, \quad A=\left(\begin{array}{rrr}
0 & 0 & 0 \\
0 & -\omega & 0 \\
0 & 0 & \omega
\end{array}\right), \quad B=\left(\begin{array}{ccc}
0 & -\phi / 2^{1 / 2} & \phi / 2^{1 / 2} \\
\phi / 2^{1 / 2} & \omega_{\xi} / 2 & -\omega_{\xi} / 2 \\
-\phi / 2^{1 / 2} & \omega_{\xi} / 2 & -\omega_{\xi} / 2
\end{array}\right),
$$

where $\phi=(s / q)^{1 / 2}$ and $\omega=(p q)^{1 / 2}$. It is now easy to verify that $L_{1} y$ can be

(12) If the deficiency indices are both zero than $l_{a}=p=m_{b}$ and $l_{b}=n=m_{a}$; hence $d_{a}=0=d_{b}$ and both $\mathfrak{V}_{a}$ and $\mathfrak{D}_{b}$ consist only of the zero vector. In fact suppose $l_{b}>n$. Then in the notation introduced at the end of $\S 3$, there exist subspaces $N_{a}$ and $N_{b}$ of the solution space to (3.1) such that $N_{a} \subset F_{a}$ is of dimension $p, N_{b} \subset F_{b}$ is of dimension $n$, and together $N_{a}$ and $N_{b}$ span the solution space. If $F_{b}$ is of dimension $>n$, there is a nontrivial solution $y=y_{a}+y_{b}, y_{a} \in N_{a}$, $y_{b} \in N_{b}$, belonging to $F_{b}$ but not to $N_{b}$. Consequently $y_{a} \in F_{a} \cap F_{b}$ and this is impossible if the deficiency indices are zero. 
obtained from $M_{1} z$ by the simple transformation $\eta^{1}=\zeta^{1}, \eta^{2}=\zeta^{3}, \eta^{3}=\zeta^{2}$. From this it follows that $\lambda \mathfrak{y}-\mathfrak{R}_{1} \mathfrak{y}=\theta$ and $\lambda_{z}-\mathfrak{M}_{1\}}=\theta$ have the same number of linearly independent solutions. Thus the deficiency indices of $\mathfrak{R}_{0}$ will be equal by Theorem 8.3 and hence according to Theorem 8.4 each conservative restriction of $R_{1}$ generates a group of unitary operators.

9. Perturbation theory. With the aid of a perturbation theory for semigroups of operators (see R. S. Phillips [12]), we now treat a somewhat more elaborately coupled system than that considered in $\$ 7$. The perturbation theory applies, in particular, to operators $\mathfrak{R}+\mathfrak{B}$, where $\mathfrak{R}$ is a dissipative operator defined as in Theorem 7.1 and $\mathfrak{P}$ is given by

$$
\mathfrak{B}=\left(\mathfrak{B}_{i j}\right) \text {, }
$$

$$
i, j=1,0,2 \text {; }
$$

here the elements of $\mathfrak{B}_{00}$ are taken to be bounded measurable functions on $(a, b)$, the elements of $\mathfrak{P}_{10}$ and $\mathfrak{P}_{02}$ are taken to be functions of $L_{2}(a, b)$, the elements of $\mathfrak{B}_{01}$ and $\mathfrak{P}_{20}$ are taken to be integral operators of the form

$$
\mathfrak{P}_{i 0, k \eta^{0}}=\int_{a}^{b} \mathfrak{p}_{i 0, k}(\xi) \mathfrak{\eta}^{0}(\xi) d \xi, \quad i=0,1,
$$

where $\mathfrak{p}_{i 0, j k} \in L_{2}(a, b)$, and the $\mathfrak{B}_{i j}, i, j=1,2$, are matrix transformations on the $\mathfrak{Z}^{i}$ space to the $\mathfrak{Y}^{i}$ space. It is clear that $\mathfrak{B}$ is a bounded linear transformation on $\mathfrak{S}$ to itself. We further impose a dissipative condition on $\mathfrak{B}$, namely,

$$
\langle\mathfrak{P} \mathfrak{y}, \mathfrak{y}\rangle+\langle\mathfrak{y}, \mathfrak{B} \mathfrak{y}\rangle \leqq 0 \quad \text { for all } \mathfrak{y} \in \mathfrak{G} \text {. }
$$

According to the above cited perturbation theory, the operator $\mathfrak{Q}+\mathfrak{B}$ generates a strongly continuous semi-group of linear bounded operators. Actually this is true even when $\mathfrak{P}$ does not satisfy the condition (9.2). However if this condition is met, then we have

THEOREM 9.1. If $\mathbb{R}$ is a dissipative restriction of $\Omega_{1}$ defined as in Theorem 7.1 and if $\mathfrak{B}$ satisfies $(9.1)$, then $\mathfrak{R}+\mathfrak{B}$ with $\mathfrak{D}(\mathfrak{R}+\mathfrak{B})=\mathfrak{D}(\mathfrak{R})$ generates a strongly continuous semi-group of contraction operators.

Proof. For $\lambda>\|\mathfrak{B}\|$ one can prove that $\lambda \in \rho(\mathfrak{R}+\mathfrak{B})$ (see [12, Lemma 3.1]). Thus given an arbitrary $\mathfrak{f} \in \mathfrak{S}$, we can set $\mathfrak{y}=\mathfrak{N}(\lambda ; \mathfrak{R}+\mathfrak{B}) \mathfrak{f}$, in which case $\mathfrak{y} \in \mathfrak{D}(\mathfrak{R})$ and

$$
\lambda \mathfrak{y}-\mathfrak{l} \mathfrak{y}-\mathfrak{B y}=\mathfrak{f} .
$$

The first relation in (7.14) implies that

$$
2 \lambda\langle\mathfrak{y}, \mathfrak{y}\rangle-\langle\mathfrak{P} \mathfrak{y}, \mathfrak{y}\rangle-\langle\mathfrak{y}, \mathfrak{P} \mathfrak{y}\rangle \leqq\langle\mathfrak{y}, \mathfrak{f}\rangle+\langle\mathfrak{f}, \mathfrak{y}\rangle+\left.\left(\mathfrak{A}_{00} \mathfrak{y}^{0}, \mathfrak{y}^{0}\right)\right|_{a-} ^{b+},
$$

and making use of (9.2) together with the fact that $y \in \mathfrak{D}(\Omega)$ satisfies a dissipative boundary condition, we obtain $\lambda\|\mathfrak{y}\| \leqq\|f\|$. In other words

$$
\lambda\|\Re(\lambda ; \mathfrak{R}+\mathfrak{B})\| \leqq 1
$$


for all sufficiently large $\lambda$. Thus the Hille-Yosida criterion is satisfied by $\mathfrak{R} \mathfrak{B}$ and it follows that this operator generates a strongly continuous semigroup of contraction operators.

We note that if $i \Omega$ is self-adjoint and $i \mathfrak{B}$ is a bounded symmetric operator, then $i(\mathfrak{R}+\mathfrak{B})$ is again self-adjoint so that $\mathfrak{R}+\mathfrak{B}$ generates a group of unitary operators. On the other hand, if $\&$ is merely a conservative restriction of a conservative operator which generates a semi-group and if $i \mathfrak{B}$ is a symmetric bounded operator, then $\mathfrak{R}+\mathfrak{B}$ generates a semi-group of isometries. The proof of the latter assertion is essentially the same as that of Theorem 8.1 and is omitted.

\section{REFERENCES} Hill, 1955.

1. E. A. Coddington and N. Levinson, Theory of ordinary differential equations, McGraw-

2. G. Doetsch, Theorie und Anwendung der Laplace-Transformation, Berlin, 1937.

3. K. O. Friedrichs, Symmetric hyperbolic linear differential equations, Communications on Pure and Applied Mathematics, vol. 7 (1954) pp. 345-392.

4. K. O. Friedrichs and H. Lewy, Über fortsetzbare Anfangsbedingungen bei hyperbolischen Differentialgleichungen in drei Veränderlichen, Nachr. Ges. Wiss. Göttingen No. 26 (1932) pp. 135-143.

5. J. Hadamard, Sur l'intégrale résiduelle, Bull. Soc. Math. France, vol. 28 (1900) pp. 69-90.

6. Günter Hellwig, Anfangs- und Randwertprobleme bei partiellen Differentialgleichungen von wechselndem Typus auf den Rändern, Math. Zeit. vol. 58 (1953) pp. 337-357.

7. Einar Hille and R. S. Phillips, Functional analysis and semi-groups, Amer. Math. Soc. Colloquium publications, vol. XXXI, to be published in 1957.

8. K. Kodaira, On ordinary differential equations of any even order and the corresponding eigenfunction expansions, Amer. J. Math. vol. 72 (1950) pp. 502-544.

9. W. Mächler, Laplacesche Integraltransformation und Integration partieller Differentialgleichungen vom hyperbolischen und parabolischen Typus, Comment. Math. Helv. vol. 5 (1933) pp. 256-304.

10. J. von Neumann, Allgemeine Eigenwerttheorie Hermitescher Funktionaloperatoren, Math. Ann. vol. 102 (1929) pp. 49-131. 573.

11. - Über einen Satz von Herrn M. H. Stone, Ann. of Math. vol. 33 (1932) pp. 567-

12. R. S. Phillips, Perturbation theory for semi-groups of linear operators, Trans. Amer. Math. Soc. vol. 74 (1953) pp. 199-221.

13. — The adjoint semi-group, Pacific J. Math. vol. 5 (1955) pp. 269-283.

14. M. Plancherel, Sur le rôle de la transformation de Laplace dans l'intégration d'une classe des problèmes mixtes du type hyperbolique et sur les développements en série d'un couple des fonctions arbitraires, C. R. Acad. Sci. Paris vol. 186 (1928) pp. 351-353.

15. F. Rellich, Spectral theory of a second order ordinary differential operator, Lectures at the New York University, 1950.

16. Kôsaku Yosida, On Cauchy's problem in the large for wave equations, Proc. of the Japan Academy vol. 28 (1952) pp. 396-403.

17. — An operator-theoretical integration of the wave equation, J. Math. Soc. Japan vol. 8 (1956) pp. 79-92.

University of Southern California, Los Angeles, Calif. 